\title{
A natural single-guide RNA repurposes Cas9 to autoregulate CRISPR-Cas expression
} Rachael E. Workman ${ }^{\# 1}$, Teja Pammi ${ }^{\# 1}$, Binh T. K. Nguyen ${ }^{1}$, Leonardo W. Graeff ${ }^{1}$, Erika Smith ${ }^{2}$, Suzanne M. Sebald ${ }^{1}$, Marie J. Stoltzfus ${ }^{1}$, Joshua W. Modell*1\%

${ }^{1}$ Department of Molecular Biology \& Genetics, Johns Hopkins University School of Medicine, Baltimore, MD 21205, USA

${ }^{2}$ Department of Biological Chemistry, Johns Hopkins University School of Medicine, Baltimore, MD 21205, USA

\#These authors contributed equally

*Correspondence: jmodell1@jhmi.edu

\%Lead contact

\section{SUMMARY}

CRISPR-Cas systems provide their prokaryotic hosts with acquired immunity against viruses and other foreign genetic elements, but how these systems are regulated to prevent auto-immunity is poorly understood. In type II CRISPR-Cas systems, a transactivating CRISPR RNA (tracrRNA) scaffold functions together with a CRISPR RNA (crRNA) guide to program Cas9 for the recognition and cleavage of foreign DNA targets. Here, we show that a long-form tracrRNA performs an unexpected second function by folding into a natural single guide that directs Cas 9 to transcriptionally repress its own promoter. Further, we demonstrate that $P_{\text {cas9 }}$ serves as a critical regulatory node; de-repression causes a dramatic induction of Cas genes, crRNAs and tracrRNAs resulting in a 3,000-fold increase in immunization rates against unrecognized viruses. Heightened immunity comes at the cost of increased auto-immune toxicity, demonstrating the critical importance of the controller. Using a bioinformatic analysis, we provide evidence that tracrRNA-mediated autoregulation is widespread in type II CRISPRCas systems. Collectively, we unveil a new paradigm for the intrinsic regulation of CRISPR-Cas systems by natural single guides, which may facilitate the frequent horizontal transfer of these systems into new hosts that have not yet evolved their own regulatory strategies.

\section{KEYWORDS}

CRISPR-Cas; regulation; Cas9; tracrRNA; natural single guide; bacteriophage

\section{INTRODUCTION}

All immune systems must distinguish "self" from "non-self" in order to provide potent activity against diverse pathogen-associated antigens while avoiding autoimmunity against the more abundant molecular catalog of the host. To help ensure specificity against foreign threats, the vertebrate adaptive immune system is a tightly regulated cellular network that undergoes spatiotemporal activation and expansion in response to novel or remembered antigens ${ }^{1}$. CRISPR-Cas systems provide bacteria and archaea with immunological memories of foreign nucleic acids, thereby protecting their prokaryotic hosts from viruses ${ }^{2}$, plasmids $^{3}$, and other 
mobile genetic elements ${ }^{4,5}$. How CRISPR-Cas immune systems are regulated within their singlecelled hosts is not well understood.

Immunological memories are encoded within CRISPR loci as short, roughly $30 \mathrm{bp}$ DNA "spacers" which are derived from segments of foreign nucleic acids ${ }^{2}$. These spacers are located within CRISPR arrays between similarly-sized repeating sequences or "repeats" 6 . CRISPR immunity occurs in three stages. During "adaptation," spacers are acquired from foreign agents and incorporated into the CRISPR array at the end proximal to a conserved "leader" sequence, causing the duplication of the leader-proximal repeat ${ }^{2}$. Under laboratory conditions, adaptation is a rare event, occurring in roughly $1 \mathrm{E}-5$ to $1 \mathrm{E}-7$ cells ${ }^{7,8}$. During "biogenesis," the CRISPR array is transcribed as one long precursor CRISPR RNA (pre-crRNA), which is cleaved within repeats to produce individual CRISPR RNAs (crRNAs), each containing a single spacer ${ }^{9-11}$. Finally, during "interference", crRNAs direct effector Cas proteins to matching foreign targets ${ }^{12-14}$, where they perform distinct catalytic activities depending on the CRISPR-Cas family and sub-type ${ }^{15}$. The type II-A CRISPR-Cas system from Streptococcus pyogenes encodes four Cas proteins, expressed as a single operon, all of which are required for adaptation (Fig. 1A) ${ }^{7}$. However, Cas 9 alone performs interference by introducing double-strand breaks into crRNA-specified DNA targets or "protospacers"16,17. To prevent the CRISPR system from cleaving its own spacers, Cas9 targeting requires a $5^{\prime}$-NGG-3' protospacer-adjacent motif (PAM) ${ }^{18,19}$ which is absent in the spaceradjacent repeats within the CRISPR array. Nonetheless, at high concentrations, Cas 9 can cleave off-target genomic loci suggesting that additional PAM-independent regulatory mechanisms may be required to avoid auto-immunity.

In addition to the pre-crRNA, the $S$. pyogenes CRISPR-Cas system harbors a second noncoding RNA, the tracrRNA, which functions during all three stages of CRISPR immunity ${ }^{7,11}$. While its role during adaptation is unclear, during biogenesis, the tracrRNA base-pairs with repeatderived sequences within the pre-crRNA, and the tracrRNA:pre-crRNA duplex is then cleaved by the host factor RNaselll within each repeat, producing individual crRNAs and a processed tracrRNA $\left(\operatorname{tr}_{\mathrm{p}}\right)^{11}$ (Fig. S1). During interference, the mature targeting complex (Cas9:trp:crRNA) facilitates target recognition and cleavage. Many CRISPR editing technologies employ an artificial single-guide RNA (sgRNA) in which $\operatorname{tr}_{p}$ and crRNA are fused with a 5'-GAAA-3' tetraloop ${ }^{13}$. In S. pyogenes, the tracrRNA is transcribed from two promoters producing a long $\left(\operatorname{tr} r_{L}\right)$ and short $\left(t r_{S}\right)$ form, both of which contain the RNaselll processing site (Fig. 1B, S1) ${ }^{11}$. Because trs alone can mediate crRNA biogenesis and interference in vivo and in vitro, it remains unclear what role tr. plays and why two tracrRNA forms are produced.

How are CRISPR systems regulated within their bacterial hosts to prevent autoimmunity and enhance targeting of foreign agents? Several studies have demonstrated transcriptional and post-transcriptional CRISPR-Cas regulation in response to extracellular and intracellular cues $^{20}$, including phage infection ${ }^{21-25}$, quorum sensing ${ }^{26,27}$, membrane stress ${ }^{28-31}$, metabolic status $^{21,32,33}$ and surface association ${ }^{34}$. In many cases where the regulatory mechanisms are known, host-encoded transcription factors interact with CRISPR-Cas promoters $32,33,35,36$, although several studies have shown that archaeal type I-A systems can be intrinsically controlled by dedicated Cas-encoded transcription factors ${ }^{25,37-40}$. Given that CRISPR systems are frequently horizontally transferred in the wild ${ }^{41-43}$, these intrinsic controllers could help prevent autoimmune toxicity ${ }^{44-47}$ in a new host that has not had time to evolve its own regulatory 
strategy. However, few CRISPR loci - and no type II loci - encode dedicated transcription factors, and it is unclear whether alternative mechanisms of intrinsic regulation exist.

Here, we identify a novel mechanism of intrinsic control, whereby a long-form tracrRNA ( $\operatorname{tr} \mathrm{L}$ ) enables Cas9 to autoregulate type II CRISPR-Cas systems. Using a transposon screen, we identify $\operatorname{tr}_{L}$ as a potent inhibitor of CRISPR immunity. We find that $\operatorname{tr}_{L}$ folds into a natural single guide RNA by providing the structural elements normally contributed by the crRNA. In place of a spacer, tr $r_{L}$ contains a truncated 11 nt targeting sequence that directs Cas 9 to transcriptionally silence its own promoter. We show that $\mathrm{P}_{\text {cas }}$ is a critical, system-wide regulatory node that controls the RNA levels of all CRISPR-Cas components. De-repression is a double-edged sword; a 3,000-fold increase in phage defense is accompanied by the deleterious effects of increased auto-immunity. Finally, we conduct a bioinformatic analysis and observe conservation of tracrRNA regulation in many type II-A CRISPR loci. Our work highlights an unexpected function for tracrRNAs and a novel form of intrinsic control within the CRISPR-Cas module, which may facilitate the frequent horizontal transfer of CRISPR systems between hosts in the wild.

\section{RESULTS}

\section{Tn-seq reveals a tracrRNA mutant with enhanced CRISPR immunity}

We initially sought to identify bacterial genes involved in CRISPR-Cas immunity through an unbiased genetic screen. In our model system, Staphylococcus aureus cells that do not encode a native CRISPR-Cas system harbor the $S$. pyogenes type II CRISPR-Cas system on a medium or low-copy plasmid ${ }^{7}$. The $S$. aureus host provides genetic tractability, an assortment of well-characterized phages, and a compatible cellular environment for the $S$. pyogenes CRISPR-Cas system. Furthermore, given that CRISPR-Cas systems are frequently horizontally transferred between bacterial hosts in the wild, our heterologous expression system serves as a model for a recent transfer event.

To create an unbiased library of genomic mutants, we introduced the transposon Tn917 ${ }^{48}$ into $S$. aureus cells carrying the $S$. pyogenes CRISPR-Cas system on a medium-copy plasmid. The CRISPR system in these cells contained a minimal "naïve" CRISPR array harboring a single CRISPR repeat and no spacers. Therefore, protection against phages required all three stages of CRISPR immunity, beginning with the acquisition of a new spacer. We next infected this library with the bacteriophage $\phi N M 4 \gamma 4^{7}$ at a multiplicity of infection (MOI) of 1 for 24 hours and identified mutants that were enriched or depleted relative to an un-infected control by deep sequencing $\mathrm{Tn}$ insertion sites $\left(\mathrm{Tn}-\mathrm{seq}^{49}\right)$. Mutations that are enriched during the infection represent enhancers of CRISPR immunity, while those that are depleted represent CRISPR repressors.

To our surprise, the transposon insertions that most strongly enhanced CRISPR immunity were within the CRISPR locus itself, spanning the $5^{\prime}$ end and promoter of $\operatorname{tr}_{\mathrm{L}}$ (Fig. 1B, S2A, Table S1). To validate the mutant phenotype, we isolated a single phage-resistant colony and confirmed the presence of a transposon insertion within the promoter of $\operatorname{tr}_{L}\left(\operatorname{tr}_{L}:: \operatorname{Tn}\right)$ and a newly acquired spacer matching $\phi N M 4 \gamma 4$. We next replaced the $\phi N M 4 \gamma 4$-targeting CRISPR array with a single repeat in order to test the effects of $\operatorname{tr}_{L}:: T n$ on a 'naïve' CRISPR system with no immunological history. In liquid and semi-solid phage immunity assays, we found that $\operatorname{tr}_{L}:: \operatorname{Tn}$ enhances CRISPR immunity by roughly 100 -fold, similar to a previously identified mutation in 
cas9 $\left(\right.$ hcas9) ${ }^{47}$ that boosts spacer acquisition rates (Fig. 1C-D, S2B). To determine whether $\operatorname{tr}_{L}:: T n$ is a gain or loss-of-function mutation, we deleted a 52 bp region spanning the CRISPR immunity-enhancing transposon insertion sites found by Tn-seq, which includes the promoter and 19 nucleotides at the $5^{\prime}$ end of $\operatorname{tr}_{L}$, generating $\Delta \operatorname{tr}_{L}$ (Fig. 1B). Strikingly, $\Delta \operatorname{tr}_{L}$ significantly enhances immunity to $\phi N M 4 \gamma 4$ by roughly 30 -fold relative to hcas 9 and $\operatorname{tr}_{L}:: T n$ and over 3,000fold relative to the wild-type system (Fig. 1C-D, S2B). To test whether expression of $\operatorname{tr}$ in trans can complement the $\Delta \operatorname{tr}_{L}$ phenotype, we cloned the tracrRNA locus onto a second plasmid and introduced two $\mathrm{T}>\mathrm{C}$ mutations in nucleotides 71 and 76 of $\operatorname{tr}$, which constitute critical residues in the -10 promoter element of $\mathrm{P}_{\mathrm{trs}}$ (Fig. S2D), effectively eliminating $\mathrm{tr}_{\mathrm{s}}$ expression (Fig. S2E). Expression of the double mutant, hereafter referred to as $\operatorname{tr}_{\mathrm{L}}{ }^{*}$, restored low levels of immunity to $\Delta \operatorname{tr}_{\mathrm{L}}$ cells (Fig. $1 \mathrm{E}, \mathrm{S} 2 \mathrm{C}$ ), indicating that (i) $\Delta \operatorname{tr}_{\mathrm{L}}$ is a loss-of-function allele and (ii) tr inhibits CRISPR immunity.

\section{$\operatorname{tr}_{L}$ inhibits multiple stages of CRISPR immunity}

The enhanced immunity of $\Delta \operatorname{tr}_{L}$ could be due to increased rates of CRISPR adaptation, biogenesis and/or interference. We therefore sought to test the $\Delta \operatorname{tr}_{L}$ mutant in assays that measure specific stages of CRISPR immunity. To test adaptation independently of subsequent steps, we electroporated a $60 \mathrm{bp}$ amplicon into cells harboring a naïve wild-type or $\Delta \operatorname{tr}_{L} \mathrm{CRISPR}$ system. The amplicon contained a single candidate protospacer with a 5'-NGG-3' PAM, which Cas9 recognizes during spacer selection ${ }^{7}$. Adaptation events were then measured by semiquantitative PCR with a forward primer within the leader of the CRISPR array and a spacerspecific reverse primer matching the NGG-adjacent electroporated DNA sequence (Fig. 2A). Because this experiment was done in the absence of phage, the proportion of cells harboring the new spacer should solely reflect adaptation rates and not differences in phage defense resulting from biogenesis and interference. Indeed, newly acquired spacers were readily observed in $\Delta \operatorname{tr}_{\mathrm{L}}$ cells but were below the limit of detection in cells with the wild-type CRISPR system (Fig. 2B). In parallel, we performed a second, phage-independent assay in which overexpression of the adaptation genes cas1, cas2 and csn2 enables spacer acquisition from the host genome and resident plasmids (Fig. S3A). Again, $\Delta \operatorname{tr}_{\mathrm{L}}$ cells showed elevated adaptation rates compared to wild-type, together indicating that $\operatorname{tr}_{L}$ impairs spacer acquisition.

To determine whether $\operatorname{tr}_{L}$ inhibits CRISPR biogenesis and/or interference, we programmed wild-type or $\Delta \operatorname{tr}_{L}$ CRISPR loci with a spacer targeting $\phi N M 4 \gamma 4$ and tested phage defense using both a plaque-formation unit (PFU) assay on semi-solid agar and a liquid cell growth assay in a 96-well plate reader. On a medium-copy plasmid, the wild-type CRISPR system defends well against $\phi N M 4 \gamma 4$, providing a 5-log reduction in PFUs and supporting bacterial growth even at an $\mathrm{MOI}$ of 300 (Fig. 2C-D, S3B). In this context, the $\Delta \operatorname{tr}_{\mathrm{L}}$ mutation did not provide an added benefit. On a low-copy plasmid, the wild-type system provides only a 1log decrease in phage PFUs and cannot support bacterial growth even at a modest $\mathrm{MOI}$ of 10 (Fig. 2C, 2E, S3C). Here, the $\Delta \operatorname{tr}_{\mathrm{L}}$ mutation significantly enhances phage defense, providing an additional 2-log decrease in PFUs and supporting robust bacterial growth in liquid culture at an $\mathrm{MOI}$ of 10 . Collectively, these results demonstrate that $\operatorname{tr}_{\mathrm{L}}$ negatively regulates multiple stages of CRISPR immunity. 


\section{trL causes widespread down-regulation of all CRISPR-Cas RNAs}

$\operatorname{tr}_{L}$ could inhibit CRISPR immunity by affecting the levels or activity of one or more CRISPR-Cas components. To measure CRISPR-Cas expression levels, we prepared RNA from cells harboring a wild-type or $\triangle \operatorname{tr}_{L}$ CRISPR system and performed Northern blots for crRNAs and tracrRNAs, qPCR using primer pairs distributed throughout the Cas operon as well as RNAseq. In the absence of $\operatorname{tr}_{L}$, the levels of $\operatorname{tr}_{S}$, $\operatorname{tr}_{\mathrm{P}}$ and processed $c r R N A s$ were all dramatically enhanced (Fig. 3A). Similarly, the protein-coding Cas genes were induced by 30 to 50 -fold (Fig. 3B, S4A-C), and Cas 9 protein levels showed a corresponding increase by Western blot (Fig. 3C). Unprocessed pre-crRNA levels as measured by qPCR increased only modestly in $\Delta \operatorname{tr}_{\text {L }}$ cells, likely due to read-through from the Cas gene operon (Fig. 3B, compare 'csn2_cr' and 'pre-crRNA' primer pairs). The increase in mature crRNA levels observed by Northern blot are therefore likely due to enhanced pre-crRNA processing owing to the increased abundance of Cas9 and/or trs. Collectively, these results demonstrate that $\operatorname{tr}_{L}$ has strong and wide-ranging inhibitory effects on the expression of all CRISPR-Cas RNAs.

\section{$\operatorname{tr}_{L}$ directs Cas9 to transcriptionally repress the Cas gene operon}

The CRISPR-Cas system is transcribed from four promoters: $P_{t r L}$ and $P_{t r s}$ transcribe the tracrRNAs, $P_{\text {cas }}$ transcribes the Cas gene operon, and $P_{c r}$ transcribes the CRISPR array (Fig. $1 A$ ). We next asked whether $\operatorname{tr}_{L}$ downregulates CRISPR-Cas RNA levels by transcriptionally repressing any of these promoters. Cells expressing Cas 9 and wild-type tracrRNA or $\Delta \operatorname{tr}$ but none of the other CRISPR-Cas elements were transformed with a second plasmid harboring a transcriptional GFP fusion to each CRISPR-Cas promoter. Cas9 and tracrRNA inhibited $P_{\text {cas9 }}$ activity by roughly 50-fold but had no effect on the other promoters (Fig. 4A), consistent with the Cas gene mRNA induction observed by qRT-PCR (Fig. 3B) and RNAseq (Fig. S4B). Furthermore, these results indicated that Cas9 and $\operatorname{tr}_{L}$ are sufficient for repression as cas1, cas2, csn2 and crRNAs were not required.

Strikingly, inspection of $\mathrm{P}_{\text {cas9 }}$ revealed an 11-nucleotide match between the $5^{\prime}$ end of $\operatorname{tr}$ and a region beginning 2 bp downstream from the $P_{\text {cas }}$ transcriptional start site (TSS) (Fig. S5A). A 5'-NGG-3' PAM, which licenses Cas9 to bind to DNA targets specified by crRNA guides, lies immediately $3^{\prime}$ of the sequence match within $\mathrm{P}_{\text {casg. }}$. Furthermore, while Cas9 cleaves DNA targets that match the 20 spacer-derived nucleotides with a given processed crRNA, shorter match lengths of up to 16 base pairs allow stable target binding but prevent cleavage ${ }^{50-52}$. Our results therefore suggested that $\operatorname{tr}_{L}$ could direct Cas9 to bind near the $\mathrm{P}_{\text {cas } 9}$ TSS and repress transcription while avoiding an autoimmune cleavage event.

To test this hypothesis, we individually mutated bases within the $P_{\text {cas }} 11$ bp match and downstream PAM on the $\mathrm{P}_{\text {cas9 }}$-GFP reporter plasmid (Fig. 4B, S5B). These constructs were introduced into strains harboring a second plasmid expressing Cas 9 and tracrRNA or an empty vector control. tr $r_{L}-$ mediated repression of $P_{\text {cas }}$ was abolished in both PAM mutants and greatly reduced in mutants of the 9 bases at the $3^{\prime}$ end of the putative $11 \mathrm{bp}$ target site. These data are consistent with previous studies showing that Cas9 binding absolutely requires a PAM and strongly prefers a perfect match within the 8-12 bp "seed" region at the 3'-end of the spacer. We also tested mutations in the bases flanking the target site and PAM, as well as the ' $N$ ' site of the 5'-NGG-3' PAM, which were repressed by $\operatorname{tr}_{L}$ at wild-type levels. To confirm that $\operatorname{tr}_{L}-$ mediated repression of $P_{\text {cas }}$ requires Cas9 protein, we combined a plasmid expressing tracrRNA 
and a cas 9 nonsense mutant with the $\mathrm{P}_{\text {cas }} 9$-GFP reporter. Indeed, $\operatorname{tr}_{\mathrm{L}}$-mediated $\mathrm{P}_{\text {cas }}$ repression required Cas9 (Fig. S5C) although tracrRNAs were undetectable in cas9 null mutants (Fig. S5D), likely because they are protected from degradation by Cas9 protein. Together, our results are consistent with a model in which the $5^{\prime}$ end of $\operatorname{tr}_{L}$ directs Cas 9 to bind its own promoter and repress transcription of the Cas gene operon.

\section{$\operatorname{tr}_{\mathrm{L}}$ is a natural single guide RNA}

To comprehensively probe the determinants of $\operatorname{tr}_{\mathrm{L}}$ repression, we generated $5 \mathrm{nt}$ mutations tiled throughout the tracrRNA region specific to $\operatorname{tr}_{L}$ as well as variably sized mutations in tracrRNA domains within trs with well characterized functions, including the upper stem, bulge, lower stem and nexus (Fig. 4C, S6D). Each tracrRNA mutant was introduced on a plasmid into cells harboring a second plasmid expressing both cas9 from a constitutive

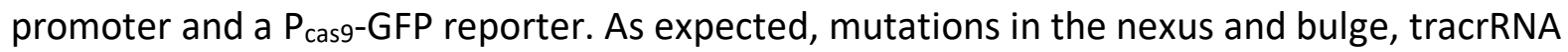
regions critical for Cas 9 binding and targeting, alleviated $t_{L}$ repression of $P_{\text {cas }}$. Mutations in the

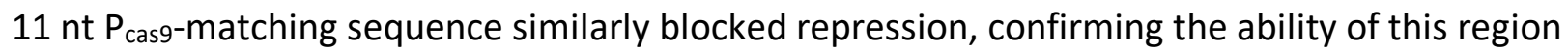
to guide $\operatorname{tr}_{\mathrm{L}}$ to its target. Curiously, mutations in the lower stem also blocked $\operatorname{tr}_{L}$ repression, while mutations in the upper stem had a milder but significant effect. The tracrRNA upper and lower stem regions normally base pair with complementary sites in the pre-crRNA repeats, enabling processing of the RNA duplex (Fig. S6B-C). However, our previous findings demonstrated that $\operatorname{tr}_{\mathrm{L}}$-mediated repression of $\mathrm{P}_{\text {cas } 9}$ occurred in the absence of crRNA (Fig. 4B), indicating that the upper and lower stems of $\operatorname{tr}_{L}$ are not occupied by their cognate crRNA residues in the Cas9:tr targeting complex. Instead, we noticed that the 12 nucleotides just

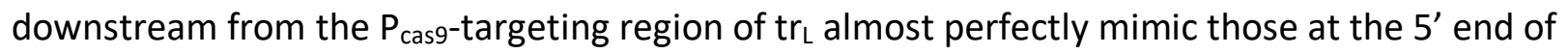
the crRNA repeat, suggesting that $\operatorname{tr}_{L}$ could fold in on itself to reconstitute the upper and lower stems (Fig. 4D). Indeed, the crRNA mimicking residues were also critical for repression (Fig. 4C), suggesting that in the absence of crRNAs, $\operatorname{tr}_{L}$ could form a natural single-guide RNA in which the

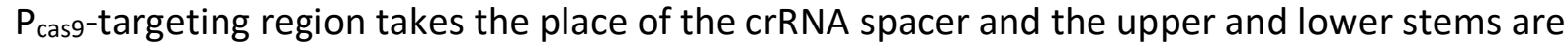
formed intramolecularly (Fig 4D, S6A).

In the putative natural single guide structure, $\operatorname{tr}_{L}$ residues $14-19$ and $113-118$ base pair to form the lower stem, and $5 \mathrm{nt}$ mutations in either strand that would prevent base pairing also disrupt tr $\mathrm{t}_{\mathrm{L}}$ repression (Fig. 4C, green bars, 4D). To interrogate this structure, we generated a construct in which both lower stem strands are simultaneously mutated to their complement, which should restore base pairing potential. Prior studies on sgRNA activity demonstrated that sequence changes within the lower stem are tolerated as long as base-pairing is maintained ${ }^{53}$. Indeed, high levels of repression were observed in the double mutant (Fig. S5E-F), supporting our model that $\operatorname{tr}_{\mathrm{L}}$ residues 14-19 mimic the crRNA repeat and form the lower stem. Another feature of the natural single guide is the large 79 nt "upper stem extension" which is replaced by an 8 nt duplex or 5'-GAAA-3' tetraloop in the crRNA and synthetic sgRNA respectively (Fig. S6A-D). To understand whether this region is dispensable for tr $\mathrm{L}_{\mathrm{L}}$ repression, we replaced the entire 79 nt segment with a 5'-GAAA-3' tetraloop and found that repression of $P_{\text {cas }}$ was maintained (Fig. S5E). Finally, to test whether Cas9 and tr $\mathrm{L}$ are sufficient to form a repressive complex on $\mathrm{P}_{\text {cas9, }}$, we performed an EMSA assay with purified Cas9, in vitro transcribed $\operatorname{tr}_{\mathrm{L}}$ and a 55 bp dsDNA derived from the $\operatorname{tr}_{\mathrm{L}}$-targeted within $\mathrm{P}_{\text {cas }}$. Indeed, Cas9: $\operatorname{tr}_{\mathrm{L}}$ bound $\mathrm{P}_{\text {cas }}$ with an 
affinity of roughly $1 \mathrm{nM}$, which was considerably tighter than the nonspecific affinity of Cas9:trL for a dsDNA derived from a non-targeted control promoter with a single PAM (Fig. 4E).

To confirm that $\operatorname{tr}_{\mathrm{L}}$ targeting is sequence-specific, owing to base-pairing within the $11 \mathrm{nt}$

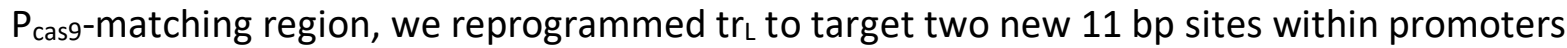
unrelated to $P_{\text {cas } 9}$ on GFP reporter plasmids (Fig. 5A). In each case, promoter activity was significantly reduced in the presence of the reprogrammed constructs but not the native tr. Next, we tested the effects of varying the tru match length and found that increasing complementarity from 11 to $13 \mathrm{bp}$ resulted in an additional 2-fold decrease in promoter activity (Fig. S7A). While a $15 \mathrm{bp}$ match did not further reduce promoter activity, we could not readily transform cells harboring $\operatorname{tr}_{L}$ constructs with GFP reporter plasmids containing a $>16 \mathrm{bp} \operatorname{tr}$ match (Fig. S7B). Previous studies showed that sgRNAs with match lengths greater than $16 \mathrm{bp}$ enable Cas 9 to cleave its target ${ }^{50-52}$, suggesting that the low transformation efficiencies we observed could be due to cleavage mediated by reprogrammed tr. To explore this possibility, we performed an in vitro Cas9 cleavage assay with $\operatorname{tr}_{\mathrm{L}}$ constructs harboring increasing match lengths to a $1 \mathrm{~kb}$ amplicon derived from the GFP reporter plasmid pCN57. Cleavage was first apparent with a $17 \mathrm{nt}$ match, and with a $19 \mathrm{nt}$ match, cleavage activity resembled that of bona fide sgRNAs (Fig. 5B). Collectively, our results suggest that tr ${ }_{\mathrm{L}}$ evolved as a natural single guide to strongly repress, but not cleave, $\mathrm{P}_{\text {cas }}$ and maintain low-levels of Cas operon expression.

\section{$\operatorname{tr}_{\mathrm{L}}$ controls a system-wide switch that is responsive to crRNA expression}

We next investigated whether the immunosuppressive effects of $\operatorname{tr}_{L}$ were solely due to repression of $\mathrm{P}_{\text {cas9, }}$, or whether $\operatorname{rr}_{L}$ could also inhibit other CRISPR functions, for instance by preventing Cas 9 and/or crRNAs from performing their canonical roles in adaptation, biogenesis, or interference. We constructed a plasmid encoding a naïve CRISPR-Cas system with a PAM mutation $\left(P_{\text {cas }}{ }^{N G C}\right.$ ) that renders $P_{\text {cas }}$ insensitive to $\operatorname{tr}_{L}$ repression (Fig. $4 A$ ). We then tested the $P_{\text {cas } 9}{ }^{N G C}$ strain in a top agar immunity assay and found high levels of phage resistance comparable to the $\Delta \operatorname{tr}_{L}$ mutant (Fig $6 \mathrm{~A}$ ), indicating that transcriptional repression is the primary if not sole inhibitory function of $\operatorname{tr}_{L}$. As expected, despite the presence of $\operatorname{tr}_{L}, \mathrm{P}_{\text {cas }}{ }^{N G C}$ cells overexpress Cas9 at $\Delta \operatorname{tr}_{\mathrm{L}}$ levels (Fig. S8A). Interestingly, this construct also phenocopies $\Delta \operatorname{tr}_{\mathrm{L}}$ with high levels of $\operatorname{tr}_{s}$, $\operatorname{tr}_{\mathrm{P}}$, and $\mathrm{crRNA}$, none of which are transcribed from $\mathrm{P}_{\text {cas9 }}$ (Fig. S8A). Given that tracrRNAs and crRNAs were undetectable in the absence of Cas9 (Fig. S5D), we wondered whether their abundance in $\Delta \operatorname{tr}_{L}$ and $\mathrm{P}_{\text {cas }}{ }^{N G C}$ could stem from Cas9 binding and stabilization. To test this hypothesis, we overexpressed Cas9 in cells harboring a wild-type CRISPR system and found that $\operatorname{tr}_{\mathrm{s}}$, $\operatorname{tr}_{\mathrm{p}}$, and crRNAs were all significantly upregulated (Fig. S8B). These results suggest that $\operatorname{tr}_{\mathrm{L}}$ serves as a master regulator for the entire CRISPR-Cas system by (i) directly controlling the protein-coding Cas operon through $\mathrm{P}_{\text {cas }}$ and (ii) indirectly controlling tracrRNA and crRNA levels by regulating Cas 9 abundance.

We next asked whether $\operatorname{tr}_{\mathrm{L}}$ is a specialized transcriptional repressor or whether it can also bind crRNAs like trs. To explore this possibility, we performed interference assays on cells harboring CRISPR systems expressing either $\operatorname{tr}_{\mathrm{L}}\left(\operatorname{tr}_{\mathrm{L}}{ }^{*}\right)$ or $\operatorname{trS}\left(\Delta \operatorname{tr}_{\mathrm{L}}\right)$, each programmed with the $\phi N M 4 \gamma 4$-targeting spacer NM2. Both systems were engineered to include the $\mathrm{P}_{\text {cas }}{ }^{\mathrm{NGC}}$ mutation in order to normalize Cas gene expression levels across experiments. In a top agar interference assay (Fig. S8C), or a liquid interference assay at low MOI (Fig. 6B), $\operatorname{tr}_{\mathrm{L}}$ and $\operatorname{tr}$ s provided similar 
levels of protection against $\phi N M 4 \gamma 4$. In liquid interference assays at high $\mathrm{MOI}$ (Fig. S8D), $\operatorname{tr}$ provided less protection than $\operatorname{tr}_{S}$, possibly because $\operatorname{tr}_{\mathrm{L}}$ is processed poorly (Fig. S8E), forming less of the mature targeting complex Cas9:trp:crRNA. Nonetheless, these results indicate that $\operatorname{tr}_{L}$ can hybridize with crRNAs in order to interfere with viral targets specified by the crRNA spacer.

Once bound, crRNAs occupy the upper and lower stems of $\operatorname{tr}_{L}$ preventing formation of the natural single guide (Fig. 4D, S6E). We therefore wondered whether crRNA expression interferes with $\operatorname{tr}_{L}$-mediated repression of $\mathrm{P}_{\text {cas }}$. To explore this possibility, we measured Cas 9 levels by Western blot in cells harboring a CRISPR-Cas system with no CRISPR array, a single repeat or an individual spacer from the natural S. pyogenes SF370 CRISPR array. We observed low Cas9 levels in cells without a CRISPR array, consistent with unimpeded $\mathrm{P}_{\text {cass }}$ repression by Cas9: $\operatorname{tr}_{\mathrm{L}}$ (Fig. 6C, S8F). The presence of a single repeat did not enhance Cas9 expression, indicating that a naïve pre-crRNA did not appreciably interfere with $\operatorname{tr}_{L}$ repression; however, the presence of any single spacer caused up-regulation of Cas9 (Fig. 6C, S8F). These results suggest the presence of a regulatory circuit in which crRNAs provide feedback through $\operatorname{tr}_{\llcorner}$to affect $P_{\text {cas }}$ expression. Notably, the CRISPR array is transcribed from its own promoter providing another entry point for system-wide regulation.

\section{CRISPR-Cas repression by $\operatorname{tr}_{L}$ inhibits auto-immunity}

Our results indicate that in wild-type cells, tr $t_{L}$ maintains the CRISPR-Cas system in a lowly active state. Given that inactivation of $\operatorname{tr}_{\mathrm{L}}$ leads to enhanced immunity, why does the system encode a $\mathrm{P}_{\text {cas }}$ repressor? One possibility is that constitutive expression of CRISPR-Cas components could cause autoimmunity, stemming from off-target adaptation or interference against the bacterial chromosome or resident plasmids ${ }^{44-47,54,55}$. To gauge whether cells harboring a de-repressed CRISPR system suffer a viability defect, we performed a liquid growth competition experiment. Cells harboring a wild-type or $\Delta \operatorname{tr}_{L}$ CRISPR system were mixed in equal parts and serially passaged twice a day. Each day, aliquots were plated to single colonies and

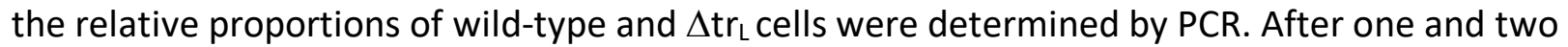
days, the number of $\Delta \operatorname{tr}_{L}$ cells dropped to $33 \%$ and $3 \%$ of their original number respectively (Fig. 6D), indicating that they suffer a growth defect compared to cells with a wild-type CRISPR system. Together, our results suggest that $\operatorname{tr}_{L}$ could repress the CRISPR system to avoid autoimmunity while allowing enough expression for some level of viral surveillance (Fig. 2B-E).

\section{TracrRNA regulation is dynamic on evolutionary timescales}

We next investigated whether $\operatorname{tr}_{L}$-mediated repression of $\mathrm{P}_{\text {cas }}$ is specific to $S$. pyogenes or conserved in other type II-A CRISPR-Cas systems. In a list of previously annotated short-form tracrRNA loci ${ }^{56}$, we examined 'branch 1 ' which contains 80 representatives from Streptococci, Listeria and Lactobacilli. We queried the presence of $\operatorname{tr}_{L}$ regulation by looking for 3 criteria: (i) an $11 \mathrm{bp}$ or greater match between a putative $\operatorname{tr}_{L}$ and a region predicted to contain the Cas 9 promoter, (ii) a 6 bp lower stem sequence ( $5^{\prime}$-GTTTTA-3') just downstream from the tr targeting site and (iii) a PAM sequence ( $5^{\prime}$-NGG-3') just downstream from the $P_{\text {cas }}$ target site. CRISPR systems that fit all three criteria, allowing for a single mismatch, were designated as " $\operatorname{tr}_{\mathrm{L}}{ }^{+\prime}$ and the others as " $\operatorname{tr}_{\mathrm{L}}{ }^{-\prime}$. Of the 80 CRISPR loci surveyed, 34 (43\%) are $\operatorname{tr}_{\mathrm{L}}{ }^{+}$including one strain with a $10 \mathrm{bp}$ match, two with a 5'-GTCTTA-3' lower stem and one with a 5'-NAG-3' PAM (Fig. 7A, Table S2). $\operatorname{tr}^{+}$genomes were not constrained to specific phylogenetic clusters but were 
distributed intermittently throughout the tree, suggesting frequent loss or gain events. Curiously, while the lower stems and PAMs showed near perfect conservation among $\operatorname{tr}_{\mathrm{L}}{ }^{+} \operatorname{loci}$, the identity of the matching sequence varied and its length ranged from 11-15 bp, indicating covariation over evolutionary timescales between the $t_{L}$ and $P_{\text {cas }}$ targeting determinants (Fig. 7A, S9). Variability was also observed in the length and sequence of the $\operatorname{tr}_{\mathrm{L}}$ upper stem extension, the precise location of the targeted sequence relative to cas 9 promoter elements, and the location of $\mathrm{P}_{\text {cas }}$ relative to the tracrRNA or cas9 coding regions (Fig. S10-11). Within $\operatorname{tr}_{L^{-}}$ loci, the intergenic space between trs and cas 9 was on average roughly 200 bp shorter than in $\mathrm{tr}_{\mathrm{L}}{ }^{+}$loci (Fig. 7B) owing to deletions that removed the targeting and/or targeted sites (Fig. S11B). In other cases, we observed $\operatorname{tr}_{L}^{-}$loci with intergenic lengths comparable to tr $_{L}^{+}$that contained one or more mismatches within the seed but retained perfect PAMs and lower stems (Fig. S11C). Our data suggests that similar seed mutants can retain intermediate levels of repression (Fig. 4B), while deletions of targeting determinants likely result in complete derepression (Fig. 4B). Collectively, our results demonstrate that $\operatorname{tr}_{\mathrm{L}}$-mediated regulation of $\mathrm{P}_{\text {casg }}$ is common in type II CRISPR-Cas systems. We speculate that individual strains have utilized, jettisoned or mutated this regulatory module in response to changes in phage predation associated with exploration of new hosts and environments.

\section{DISCUSSION}

How CRISPR-Cas systems are regulated to enhance the targeting of foreign nucleic acids while avoiding autoimmunity is a fundamental yet poorly understood aspect of CRISPR biology. In the absence of dedicated Cas-encoded transcription factors, a mechanistic understanding of whether and how type II CRISPR-Cas9 systems are regulated was unknown. Here, we report that long-form tracrRNAs are capable of forming natural single guides that direct Cas 9 to transcriptionally repress the Cas operon (Fig. 4A, S6, summarized in Fig. 8). As a result, the CRISPR-Cas system is kept in a lowly active state that is sufficient to interfere against remembered threats (Fig. 2C-E) but adapts poorly to unrecognized phages. De-repression of the system, by genetic ablation of $\mathrm{rr}_{L}$, results in a roughly 50 -fold increase in CRISPR-Cas components (Fig. 3, S4) and a 3,000-fold increase in immunization rates against an unrecognized phage (Fig. 1B-E). As with many immune systems, this hyperactivity comes at a price; cells with de-repressed systems are outcompeted by wild-type cells in the absence of a phage threat (Fig. 6D). Below, we propose that bacteria navigate these costs and benefits through (i) mutation of the regulatory controls and (ii) transient de-repression of $P_{\text {cas }}$ :tr tr. $_{\text {. }}$

Using a bioinformatic approach, we identified $\operatorname{tr}_{L}$ transcriptional regulators throughout many branches of the type II-A CRISPR-Cas tree (Fig. 7A). Moreover, we found evidence that the regulatory elements are frequently lost, suggesting that individual strains can use or lose this system according to their unique needs. In cases where the regulator is wholly absent, high basal rates of CRISPR-Cas expression could enable survival in environments with abundant phage. It is unclear whether and how these strains manage the autoimmunity associated with tr L loss. In other cases, mutations in the seed, lower stem or PAM could only partially relieve repression resulting in slightly elevated rates of basal CRISPR-Cas expression (Fig. 4B). These strains may better survive phage-replete environments while mitigating the autoimmunity associated with full tr $r_{L}$ loss. Furthermore, we speculate that seed mutants in particular could represent evolutionary stepping stones, where a second compensatory mutation could restore 
repression, resulting in the covariation we observed between the targeting and targeted sites throughout the type II-A $\operatorname{tr}_{L}$ tree. By introducing a single mutation at either site, individuals within a bacterial community can sample modifications to $P_{\text {cas9, }}$, affecting basal expression levels and/or regulatory strength, to prepare the community for changing environments. Work in our lab seeks to understand whether tr $r_{\text {- }}$-regulation extends to more distant branches of the type II tree, and whether $\operatorname{tr}_{L}$ can target other promoters, either within or beyond the CRISPR locus. In the $F$. novicida type II-B CRISPR system, tracrRNA hybridizes with a non-canonical 'scaRNA' that utilizes an $11 \mathrm{bp}$ targeting sequence to transcriptionally repress an immunostimulatory lipoprotein ${ }^{52}$ in the bacterial genome. Our work shows that scaRNAs are not required for Cas9mediated transcriptional control, greatly expanding the list of CRISPR systems with candidate regulators. Further, we show that transcriptional regulation by Cas 9 is not restricted to $F$. novicida but may be a conserved property across type II CRISPR-Cas systems.

We expect that Cas9:tr repression can also be transiently relieved in response to an unknown stimulus. We note that the configuration of the $S$. pyogenes CRISPR-Cas regulatory circuit offers several entry points for transient induction. Transcriptional changes in the activity of $P_{t r L}, P_{t r S}$ or $P_{c r}$ could each induce $P_{c a s}$ by decreasing the levels or repressive potential of $\operatorname{tr}$. In the simplest case, inhibition of $P_{t r L}$ would alleviate $P_{\text {cas }}$ repression by downregulating $\operatorname{tr} r_{L}$ itself. $P_{\text {trs }}$ activation could destabilize $\operatorname{tr}_{L}$ as it is outcompeted by trs for Cas 9 binding and protection. $P_{c r}$ induction could inactivate $\operatorname{tr}_{L}$ as the upper and lower stems are bound by accumulating precrRNAs, preventing folding of the natural single guide. Processing of $\operatorname{tr}_{L}$ by RNAselll separates the $5^{\prime}$ targeting region from the $3^{\prime}$ nexus and termination hairpins, which are required for Cas 9 binding. Therefore, cellular conditions that upregulate or activate RNAsellI could transiently induce CRISPR-Cas expression. Another intriguing candidate for post-transcriptional regulation is the $79 \mathrm{nt}$ upper stem extension of the natural single guide, which is wholly dispensable for repression (Fig. 4C) and likely to be extruded from the main body of Cas957. Secondary structural predictions of this region include a series of hairpins and loops (Fig. 4D) ${ }^{58}$ which could serve as substrates for cleavage, sequestration or re-folding by host proteins and/or small molecules.

Another candidate mechanism for transient CRISPR-Cas induction is the sequestration of Cas9:tr to a crRNA-targeted phage during an infection. In archaeal type I-A systems, the Casencoded transcription factor $\mathrm{Csa3b}$ associates with the Cascade interference complex, and the introduction of a viral target de-represses CRISPR-Cas expression by sequestering Cascade:Csa3b. It remains unclear whether this induction is necessary for viral defense or if a similar strategy could provide protection in bacteria given the short lifecycle of a bacteriophage compared to an archaeal virus. The signals and mechanisms of transient induction, especially those occurring in the native $S$. pyogenes host, are active areas of exploration in our lab.

Our results provide the first example of an intrinsic CRISPR-Cas regulator within a bacterial host. The emerging literature on CRISPR-Cas regulation in bacteria focuses on transcription factors or chaperones encoded in the genomes of hosts with type I CRISPRsystems ${ }^{20}$. In these cases, CRISPR-Cas promoters likely evolved to join existing regulons, enabling CRISPR activities to be tied to a variety of internal and environmental stimuli. Less clear is how and whether CRISPR-Cas systems are regulated following horizontal transfer into a new host, a major route of CRISPR-Cas evolution, particularly for type II systems which are present in diverse animal-associated microbiomes ${ }^{59}$. We believe that intrinsic regulators like $\operatorname{tr}_{\mathrm{L}}$ 
could facilitate horizontal transfer by dampening autoimmunity upon delivery and allowing the new bacterial host to tune CRISPR-Cas9 expression to meet its needs, through mutation or deletion of the control elements. Whether type II systems join host regulons over time, use only intrinsic control, or evolve overlapping extrinsic and intrinsic pathways are open questions. Archaeal type I-A systems are the lone other example of intrinsic regulation, mediated by a Casencoded transcription factor ${ }^{25}$. Our results show that intrinsic control does not require dedicated transcription factors and may be a more general property of CRISPR-Cas biology. It remains to be seen whether other CRISPR-Cas types have evolved their own RNA-guided intrinsic regulators, for instance using degenerate self-targeting spacers ${ }^{60}$.

Discoveries in basic CRISPR-Cas biology have continually led to new rounds of CRISPR tool development. We believe a better understanding of how $\operatorname{tr}_{L}$ is naturally regulated will facilitate the development of controllable single guides. The recent creation of a theophyllineresponsive Cas9 RNP by the fusion of an aptamer to the sgRNA upper stem ${ }^{61}$ underscores the potential for regulation to occur through the solvent-exposed $79 \mathrm{nt}$ upper stem extension of $\operatorname{tr}_{L}$.

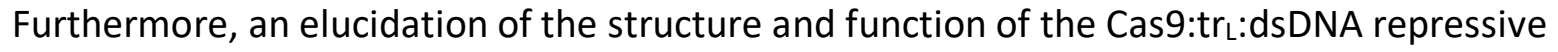
complex could inform a new generation of Cas9-based transcriptional tools. As the list of sequenced microbial genomes grows, so does the catalog of novel Cas9 orthologs, each with the possibility of new PAMs and unique cleavage activities. Our results suggest that many of these genomes might be worth a closer look for the blueprints of natural single guides.

\section{ACKNOWLEDGEMENTS}

We would like to thank Michael Laub, Luciano Marraffini, Geraldine Seydoux and Samuel Sternberg for their comments on the manuscript. We thank Karole D'Orazio and members of Rachel Green's lab for sharing technical assistance and reagents for in vitro experiments. We thank Jeremy Nathans' and Carol Greider's lab for sharing reagents and equipment. We thank David Mohr and the GRCF High Throughput Sequencing Center for assistance with NGS experiments.

\section{AUTHOR CONTRIBUTIONS}

R.E.W., T.P., B.T.K.N., L.W.G. and J.W.M. designed and executed the research studies. E.S., S.M.S., and M.J.S. assisted with plasmid construction. R.E.W. and J.W.M. wrote the manuscript.

\section{DECLARATION OF INTERESTS}

No declaration. 


\section{References}

1 Gasteiger, G. \& Rudensky, A. Y. Interactions between innate and adaptive lymphocytes. Nat Rev Immunol 14, 631-639, doi:10.1038/nri3726 (2014).

2 Barrangou, R. et al. CRISPR provides acquired resistance against viruses in prokaryotes. Science 315, 1709-1712, doi:10.1126/science.1138140 (2007).

3 Marraffini, L. A. \& Sontheimer, E. J. CRISPR interference limits horizontal gene transfer in staphylococci by targeting DNA. Science 322, 1843-1845, doi:10.1126/science.1165771 (2008).

4 Makarova, K. S. et al. Evolutionary classification of CRISPR-Cas systems: a burst of class 2 and derived variants. Nat Rev Microbiol 18, 67-83, doi:10.1038/s41579-019-0299-x (2020).

5 Marraffini, L. A. CRISPR-Cas immunity in prokaryotes. Nature 526, 55-61, doi:10.1038/nature15386 (2015).

6 Mojica, F. J. \& Rodriguez-Valera, F. The discovery of CRISPR in archaea and bacteria. FEBS J 283, 3162-3169, doi:10.1111/febs.13766 (2016).

7 Heler, R. et al. Cas9 specifies functional viral targets during CRISPR-Cas adaptation. Nature 519, 199-202, doi:10.1038/nature14245 (2015).

8 Hynes, A. P., Villion, M. \& Moineau, S. Adaptation in bacterial CRISPR-Cas immunity can be driven by defective phages. Nat Commun 5, 4399, doi:10.1038/ncomms5399 (2014).

9 Brouns, S. J. et al. Small CRISPR RNAs guide antiviral defense in prokaryotes. Science 321, 960-964, doi:10.1126/science.1159689 (2008).

10 Carte, J., Wang, R., Li, H., Terns, R. M. \& Terns, M. P. Cas6 is an endoribonuclease that generates guide RNAs for invader defense in prokaryotes. Genes Dev 22, 3489-3496, doi:10.1101/gad.1742908 (2008).

11 Deltcheva, E. et al. CRISPR RNA maturation by trans-encoded small RNA and host factor RNase III. Nature 471, 602-607, doi:10.1038/nature09886 (2011).

12 Gasiunas, G., Barrangou, R., Horvath, P. \& Siksnys, V. Cas9-crRNA ribonucleoprotein complex mediates specific DNA cleavage for adaptive immunity in bacteria. Proc Natl Acad Sci U S A 109, E2579-2586, doi:10.1073/pnas.1208507109 (2012).

13 Jinek, M. et al. A programmable dual-RNA-guided DNA endonuclease in adaptive bacterial immunity. Science 337, 816-821, doi:10.1126/science.1225829 (2012).

14 Jore, M. M. et al. Structural basis for CRISPR RNA-guided DNA recognition by Cascade. Nat Struct Mol Biol 18, 529-536, doi:10.1038/nsmb.2019 (2011).

15 Plagens, A., Richter, H., Charpentier, E. \& Randau, L. DNA and RNA interference mechanisms by CRISPR-Cas surveillance complexes. FEMS Microbiol Rev 39, 442-463, doi:10.1093/femsre/fuv019 (2015).

16 Garneau, J. E. et al. The CRISPR/Cas bacterial immune system cleaves bacteriophage and plasmid DNA. Nature 468, 67-71, doi:10.1038/nature09523 (2010).

17 Sapranauskas, R. et al. The Streptococcus thermophilus CRISPR/Cas system provides immunity in Escherichia coli. Nucleic Acids Res 39, 9275-9282, doi:10.1093/nar/gkr606 (2011).

18 Deveau, H. et al. Phage response to CRISPR-encoded resistance in Streptococcus thermophilus. J Bacteriol 190, 1390-1400, doi:10.1128/JB.01412-07 (2008). 
19 Horvath, P. et al. Diversity, activity, and evolution of CRISPR loci in Streptococcus thermophilus. J Bacteriol 190, 1401-1412, doi:10.1128/JB.01415-07 (2008).

20 Patterson, A. G., Yevstigneyeva, M. S. \& Fineran, P. C. Regulation of CRISPR-Cas adaptive immune systems. Curr Opin Microbiol 37, 1-7, doi:10.1016/j.mib.2017.02.004 (2017).

21 Agari, Y. et al. Transcription profile of Thermus thermophilus CRISPR systems after phage infection. J Mol Biol 395, 270-281, doi:10.1016/j.jmb.2009.10.057 (2010).

22 Young, J. C. et al. Phage-induced expression of CRISPR-associated proteins is revealed by shotgun proteomics in Streptococcus thermophilus. PLoS One 7, e38077, doi:10.1371/journal.pone.0038077 (2012).

23 Quax, T. E. et al. Massive activation of archaeal defense genes during viral infection. J Virol 87, 8419-8428, doi:10.1128/JVI.01020-13 (2013).

24 Fusco, S. et al. Transcriptome analysis of Sulfolobus solfataricus infected with two related fuselloviruses reveals novel insights into the regulation of CRISPR-Cas system. Biochimie 118, 322-332, doi:10.1016/j.biochi.2015.04.006 (2015).

25 He, F., Vestergaard, G., Peng, W., She, Q. \& Peng, X. CRISPR-Cas type I-A Cascade complex couples viral infection surveillance to host transcriptional regulation in the dependence of Csa3b. Nucleic Acids Res 45, 1902-1913, doi:10.1093/nar/gkw1265 (2017).

26 Patterson, A. G. et al. Quorum Sensing Controls Adaptive Immunity through the Regulation of Multiple CRISPR-Cas Systems. Mol Cell 64, 1102-1108, doi:10.1016/j.molcel.2016.11.012 (2016).

27 Hoyland-Kroghsbo, N. M. et al. Quorum sensing controls the Pseudomonas aeruginosa CRISPR-Cas adaptive immune system. Proc Natl Acad Sci U S A 114, 131-135, doi:10.1073/pnas.1617415113 (2017).

28 Ratner, H. K., Sampson, T. R. \& Weiss, D. S. I can see CRISPR now, even when phage are gone: a view on alternative CRISPR-Cas functions from the prokaryotic envelope. Curr Opin Infect Dis 28, 267-274, doi:10.1097/QCO.0000000000000154 (2015).

29 Perez-Rodriguez, R. et al. Envelope stress is a trigger of CRISPR RNA-mediated DNA silencing in Escherichia coli. Mol Microbiol 79, 584-599, doi:10.1111/j.13652958.2010.07482.x (2011).

30 Yosef, I., Goren, M. G., Kiro, R., Edgar, R. \& Qimron, U. High-temperature protein G is essential for activity of the Escherichia coli clustered regularly interspaced short palindromic repeats (CRISPR)/Cas system. Proc Natl Acad Sci U S A 108, 20136-20141, doi:10.1073/pnas.1113519108 (2011).

31 Majsec, K., Bolt, E. L. \& Ivancic-Bace, I. Cas3 is a limiting factor for CRISPR-Cas immunity in Escherichia coli cells lacking H-NS. BMC Microbiol 16, 28, doi:10.1186/s12866-0160643-5 (2016).

32 Shinkai, A. et al. Transcription activation mediated by a cyclic AMP receptor protein from Thermus thermophilus HB8. J Bacteriol 189, 3891-3901, doi:10.1128/JB.01739-06 (2007).

33 Patterson, A. G., Chang, J. T., Taylor, C. \& Fineran, P. C. Regulation of the Type I-F CRISPR-Cas system by CRP-CAMP and GalM controls spacer acquisition and interference. Nucleic Acids Res 43, 6038-6048, doi:10.1093/nar/gkv517 (2015). 
34 Borges, A. L. et al. Bacterial alginate regulators and phage homologs repress CRISPR-Cas immunity. Nat Microbiol 5, 679-687, doi:10.1038/s41564-020-0691-3 (2020).

35 Medina-Aparicio, L. et al. The CRISPR/Cas immune system is an operon regulated by LeuO, H-NS, and leucine-responsive regulatory protein in Salmonella enterica serovar Typhi. J Bacteriol 193, 2396-2407, doi:10.1128/JB.01480-10 (2011).

36 Westra, E. R. et al. H-NS-mediated repression of CRISPR-based immunity in Escherichia coli K12 can be relieved by the transcription activator LeuO. Mol Microbiol 77, 13801393, doi:10.1111/j.1365-2958.2010.07315.x (2010).

37 Liu, T. et al. Transcriptional regulator-mediated activation of adaptation genes triggers CRISPR de novo spacer acquisition. Nucleic Acids Res 43, 1044-1055, doi:10.1093/nar/gku1383 (2015).

38 Lintner, N. G. et al. The structure of the CRISPR-associated protein Csa3 provides insight into the regulation of the CRISPR/Cas system. J Mol Biol 405, 939-955, doi:10.1016/j.jmb.2010.11.019 (2011).

39 Haft, D. H., Selengut, J., Mongodin, E. F. \& Nelson, K. E. A guild of 45 CRISPR-associated (Cas) protein families and multiple CRISPR/Cas subtypes exist in prokaryotic genomes. PLoS Comput Biol 1, e60, doi:10.1371/journal.pcbi.0010060 (2005).

40 Viswanathan, P., Murphy, K., Julien, B., Garza, A. G. \& Kroos, L. Regulation of dev, an operon that includes genes essential for Myxococcus xanthus development and CRISPRassociated genes and repeats. J Bacteriol 189, 3738-3750, doi:10.1128/JB.00187-07 (2007).

41 Takeuchi, N., Wolf, Y. I., Makarova, K. S. \& Koonin, E. V. Nature and intensity of selection pressure on CRISPR-associated genes. J Bacteriol 194, 1216-1225, doi:10.1128/JB.0652111 (2012).

42 Chakraborty, S. et al. Comparative network clustering of direct repeats (DRs) and cas genes confirms the possibility of the horizontal transfer of CRISPR locus among bacteria. Mol Phylogenet Evol 56, 878-887, doi:10.1016/j.ympev.2010.05.020 (2010).

43 Godde, J. S. \& Bickerton, A. The repetitive DNA elements called CRISPRs and their associated genes: evidence of horizontal transfer among prokaryotes. J Mol Evol 62, 718-729, doi:10.1007/s00239-005-0223-z (2006).

44 Vale, P. F. et al. Costs of CRISPR-Cas-mediated resistance in Streptococcus thermophilus. Proc Biol Sci 282, 20151270, doi:10.1098/rspb.2015.1270 (2015).

45 Vercoe, R. B. et al. Cytotoxic chromosomal targeting by CRISPR/Cas systems can reshape bacterial genomes and expel or remodel pathogenicity islands. PLoS Genet 9, e1003454, doi:10.1371/journal.pgen.1003454 (2013).

46 Jiang, W. et al. Dealing with the evolutionary downside of CRISPR immunity: bacteria and beneficial plasmids. PLoS Genet 9, e1003844, doi:10.1371/journal.pgen.1003844 (2013).

47 Heler, R. et al. Mutations in Cas9 Enhance the Rate of Acquisition of Viral Spacer Sequences during the CRISPR-Cas Immune Response. Mol Cell 65, 168-175, doi:10.1016/j.molcel.2016.11.031 (2017).

48 Tomich, P. K., An, F. Y. \& Clewell, D. B. A transposon (Tn917) in Streptococcus faecalis that exhibits enhanced transposition during induction of drug resistance. Cold Spring Harb Symp Quant Biol 43 Pt 2, 1217-1221, doi:10.1101/sqb.1979.043.01.138 (1979). 
49 van Opijnen, T., Bodi, K. L. \& Camilli, A. Tn-seq: high-throughput parallel sequencing for fitness and genetic interaction studies in microorganisms. Nat Methods 6, 767-772, doi:10.1038/nmeth.1377 (2009).

50 Bikard, D. et al. Programmable repression and activation of bacterial gene expression using an engineered CRISPR-Cas system. Nucleic Acids Res 41, 7429-7437, doi:10.1093/nar/gkt520 (2013).

51 Sternberg, S. H., LaFrance, B., Kaplan, M. \& Doudna, J. A. Conformational control of DNA target cleavage by CRISPR-Cas9. Nature 527, 110-113, doi:10.1038/nature15544 (2015).

52 Ratner, H. K. et al. Catalytically Active Cas9 Mediates Transcriptional Interference to Facilitate Bacterial Virulence. Mol Cell 75, 498-510 e495, doi:10.1016/j.molcel.2019.05.029 (2019).

53 Briner, A. E. et al. Guide RNA functional modules direct Cas9 activity and orthogonality. Mol Cell 56, 333-339, doi:10.1016/j.molcel.2014.09.019 (2014).

54 Modell, J. W., Jiang, W. \& Marraffini, L. A. CRISPR-Cas systems exploit viral DNA injection to establish and maintain adaptive immunity. Nature 544, 101-104, doi:10.1038/nature21719 (2017).

55 Levy, A. et al. CRISPR adaptation biases explain preference for acquisition of foreign DNA. Nature 520, 505-510, doi:10.1038/nature14302 (2015).

56 Faure, G. et al. Comparative genomics and evolution of trans-activating RNAs in Class 2 CRISPR-Cas systems. RNA Biol 16, 435-448, doi:10.1080/15476286.2018.1493331 (2019).

57 Nishimasu, H. et al. Crystal structure of Cas9 in complex with guide RNA and target DNA. Cell 156, 935-949, doi:10.1016/j.cell.2014.02.001 (2014).

58 Mathews, D. H. et al. Incorporating chemical modification constraints into a dynamic programming algorithm for prediction of RNA secondary structure. Proc Natl Acad Sci U S A 101, 7287-7292, doi:10.1073/pnas.0401799101 (2004).

59 Chylinski, K., Makarova, K. S., Charpentier, E. \& Koonin, E. V. Classification and evolution of type II CRISPR-Cas systems. Nucleic Acids Res 42, 6091-6105, doi:10.1093/nar/gku241 (2014).

60 Ma, K. et al. cas9 Enhances Bacterial Virulence by Repressing the regR Transcriptional Regulator in Streptococcus agalactiae. Infect Immun 86, doi:ARTN e00552-17 10.1128/IAI.00552-17 (2018).

61 Kundert, K. et al. Controlling CRISPR-Cas9 with ligand-activated and ligand-deactivated sgRNAs. Nat Commun 10, 2127, doi:10.1038/s41467-019-09985-2 (2019). 


\section{FIGURE LEGENDS}

Figure 1. Tn-seq reveals a tracrRNA mutant with enhanced CRISPR immunity. A) Schematic of the $S$. pyogenes type II CRISPR-Cas system. Arrows indicate promoters. CRISPR array repeats (black boxes) and spacers (colored boxes) are shown. B) Tn-seq reads are overlaid on the corresponding tracrRNA and $\mathrm{P}_{\text {cas }}$ DNA regions. NGS reads at each site are shown as the fraction of total reads. Data is representative of biological replicates. Top panel, - phage; bottom panel, + phage. Horizontal bar indicates the region deleted in $\Delta \operatorname{tr}_{L}$. C) Cells harboring naïve CRISPR-Cas systems with the indicated mutations were infected with $\phi N M 4 \gamma 4$ at $\mathrm{MOI}=10$ and cell densities $\left(\mathrm{OD}_{600}\right)$ were measured every 10 minutes in a 96-well plate reader. hcas9, hyper-Cas9; $\operatorname{tr}_{L}:: T n$, transposon insertion in $\mathrm{P}_{\text {tracr; }}$ CRISPR', no CRISPR system. Error bars represent SEM. D-E) Naïve CRISPR-Cas mutants were infected with $\phi N M 4 \gamma 4$ at $\mathrm{MOI}=25$ in top agar, and surviving colonies with expanded CRISPR arrays were quantified. Bar graphs here and throughout show mean +/- SEM. E) An empty vector (EV) or a plasmid expressing long-form tracrRNA (tr ${ }^{*}$ ) was introduced to the indicated strains from (D).

Figure 2. Long-form tracrRNA ( $\operatorname{tr}_{L}$ ) inhibits both spacer acquisition and interference. A) Schematic of spacer acquisition assay. B) Water or a 60bp dsDNA amplicon with a single PAM was electroporated into cells with a naïve wt or $\triangle$ trL CRISPR system, and adaptation was monitored with a spacer-specific PCR. C) 10-fold dilutions of phage $\phi N M 4 \gamma 4$ were plaqued on top agar lawns containing cells with medium or low-copy CRISPR plasmids harboring the indicated spacers. NT, non-targeting spacer; $\alpha-\phi N M 4$, spacer targeting $\phi N M 4 \gamma 4$. D-E) The medium (D) and low-copy (E) strains from (C) were infected with $\phi N M 4 \gamma 4$ in liquid culture at $\mathrm{MOI}=300(\mathrm{D})$ or $\mathrm{MOI}=10(\mathrm{E})$. Cell densities $\left(\mathrm{OD}_{600}\right)$ were measured every 10 minutes in a 96-well plate reader.

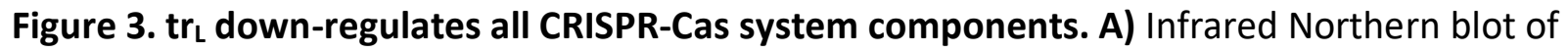
cells harboring the indicated plasmids grown to stationary phase. EV, empty vector; wt, naïve wild-type CRISPR system; $\Delta \operatorname{tr}_{L}$, naïve $\Delta \operatorname{tr}_{L}$ CRISPR system; $\operatorname{tr}_{L}{ }^{*}$, a plasmid expressing long-form tracrRNA. Membranes were probed with oligos matching the $3^{\prime}$ end of tracrRNA (top), the crRNA repeat (middle) or the 4.5S RNA (bottom, loading control). B) RT-qPCR of the indicated strains using primers within the coding region of cas9, cas1 or csn2, on either side of the crRNA promoter (csn2_cr), within the pre-crRNA leader sequence or within the processed tracrRNA $(\operatorname{tr} p)$. Fold-change was expressed as $2^{-\Delta C t}$ for each indicated primer pair relative to a rho control primer pair. Bottom panel shows the locations of the amplicons. C) Infrared Western blot of the strains in (A) using an $\alpha$-Cas9 antibody. Membranes were stained with Ponceau $S$, and the prominent band is shown as a loading control (total protein, TPN).

Figure 4. $\operatorname{tr}_{\mathrm{L}}$ is a natural sgRNA (nt-sgRNA) that directs Cas9 to repress its own promoter. A) Promoter activity was measured (fluorescence $/ O D_{600}$ ) in cells harboring a plasmid expressing GFP from the indicated promoters, and a second plasmid encoding Cas9 and the full tracrRNA locus or an empty vector. B) Promoter activity was measured as in (A) with $\mathrm{P}_{\text {cass-GFP reporter }}$ constructs harboring single mutations to the complementary base at the indicated positions. 
The asterisk indicates that the mutation at the -10 site significantly reduced basal $P_{\text {cas }}$ expression (Fig. S5B), rendering the +/- Cas9-tracr comparison inconclusive. C) Promoter activity was measured in cells harboring a plasmid expressing Cas 9 from a constitutive promoter and

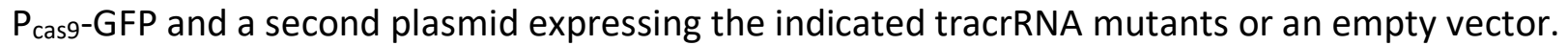
Sets of mutations were introduced in the tracrRNA plasmid at the indicated positions (relative to the TSS of $\mathrm{tr}_{\mathrm{L}}$ ) to their complementary bases. D) Putative structure of the nt-sgRNA. Orange,

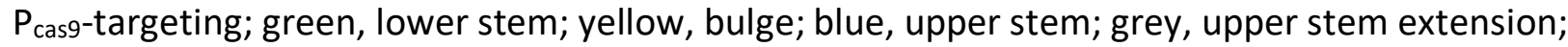
pink, nexus; purple, termination hairpins; magenta circle $\left(\operatorname{tr}_{s}\right)$, short-form tracrRNA start site. E) Electromobility shift assay (EMSA). A radiolabeled dsDNA derived from $\mathrm{P}_{\text {GFP }}$ or $\mathrm{P}_{\text {cas9 }}$ at $50 \mathrm{pM}$ was mixed with Cas9:tr RNPs at the indicated concentrations at $37^{\circ} \mathrm{C}$ for 1 hour. Reactions were run on an $8 \%$ TBE gel.

Figure 5. $\operatorname{tr}_{\mathrm{L}}$ can be reprogrammed to direct Cas9 repression or cleavage of novel targets. A) Promoter activity was measured in cells harboring the GFP reporter plasmid pJW711 or pCN57 and a second plasmid expressing Cas9 and the indicated $\operatorname{tr}_{L}$ variants. EV, empty vector; WT, the wild-type $\mathrm{P}_{\text {casg-targeting }} \mathrm{r}_{\mathrm{L}}$; $\mathrm{pRW} 20$ and $\mathrm{pRW} 22$, $\operatorname{tr}_{\mathrm{L}}$ reprogrammed to target the $11 \mathrm{bp}$ regions indicated in brown or gray bars respectively. The asterisk indicates a second pRW20 binding site within pCN57 containing a single mismatch at the -9 seed position relative to the PAM. B) In vitro cleavage assay. $30 \mathrm{nM}$ sgRNA or tr, $33.3 \mathrm{nM}$ Cas9 and a $3 \mathrm{nM} 1 \mathrm{~kb}$ amplicon from pCN57 were incubated at RT for 10 min and cleavage products were run on a 1.5\% agarose gel. WT, $\operatorname{tr}_{\mathrm{L}}$; 11-19, $\operatorname{tr}_{L}$ variants transcribed from pRW22 with an expanded pCN57 targeting sequence of the indicated length; SG1-2, replicates of an sgRNA with a 20nt pCN57 targeting sequence.

Figure 6. $\operatorname{tr}_{L}$ is master switch that controls auto-immunity and responds to crRNA spacer identity. A) Naïve CRISPR-Cas mutants were infected with $\phi N M 4 \gamma 4$ at $\mathrm{MOI}=25$ in top agar, and surviving colonies were quantified. $\Delta \operatorname{tr}_{L}$, deletion of the long-form tracrRNA; $P_{\text {cas }}{ }^{N G C}, P A M$ mutation in the $\operatorname{tr}_{\mathrm{L}}$ target site within $\mathrm{P}_{\text {cas }}$. B) Interference assay with $\phi N M 4 \gamma 4$ at $\mathrm{MOI}=1$ with cells harboring a plasmid expressing Cas9, the $\phi N M 4 \gamma 4$-targeting spacer NM2, and the indicated tracrRNA mutants. tr ${ }^{*}{ }^{*}$, long-form tracrRNA only; $\Delta$ tr, full deletion of the tracrRNA locus. The $\operatorname{tr}_{\mathrm{L}}{ }^{*}$ strain harbored a second plasmid expressing additional $\mathrm{tr}_{\mathrm{L}}{ }^{*}$ from $\mathrm{P}_{\text {sparc2 }}$ in order to ensure levels of $\operatorname{tr}_{L}$ expression comparable to $\operatorname{tr}_{S}$ in the $\Delta \operatorname{tr}_{\mathrm{L}}$ strain. All other strains harbored a second empty vector. IPTG was added at $1 \mathrm{mM}$ to induce expression from $\mathrm{P}_{\text {sparc2 }}$. C) Chemiluminescent Western blot using an $\alpha$-Cas9 antibody on logarithmic phase cells harboring a plasmid expressing the wild-type CRISPR system with the CRISPR array deleted and a second plasmid expressing no CRISPR array (EV) a single repeat (SR) or a single spacer from the endogenous $S$. pyogenes CRISPR array (Sp1-6). Quantification of biological triplicates shown in Fig. S8F. D) Cells harboring a plasmid expressing a naïve wild-type or $\Delta \operatorname{tr}_{L}$ CRISPR system were co-cultured in a competition assay. Cells were grown to stationary phase and diluted into logarithmic phase twice per day, and each morning, cultures were plated and wild-type and $\Delta \operatorname{tr}_{L}$ cells were counted by PCR of the tracrRNA locus.

Figure 7. $\operatorname{tr}_{\mathrm{L}}$ is a conserved and dynamic feature of type II-A CRISPR-Cas systems. A) A phylogenetic tree was constructed using Cas9 protein sequences from representative type II-A 
'branch-1' genomes. Each CRISPR-Cas locus was queried for the presence of $\operatorname{tr}_{L}$-mediated regulation of $\mathrm{P}_{\text {cas }}$ (black boxes, $\operatorname{tr}_{L^{+}}$; white boxes, $\operatorname{tr}_{L^{-}}$) by looking for an 11 bp candidate targeting sequence (CTS), a 5'-GTTTTA-3' lower stem (LS) and target site 5'-NGG-3' PAM. The intergenic distance between the translation start site of Cas9 and the transcriptional start site of the short-form tracrRNA (trs-cas9) was measured and plotted as a heatmap (red boxes = longer distances, blue boxes = shorter distances). The CTS and lower stem LS are listed for each $\operatorname{tr}_{\mathrm{L}}{ }^{+}$locus, with nucleotides colored by identity. * 10 bp CTS; \#, 5'-NAG-3' PAM; \&, 5'-GTCTTA-3' LS; scale bar, average amino acid substitutions per site. Full metadata available in Table S2. B) Schematic showing the intergenic distance between $\operatorname{tr}_{\mathrm{s}}$ and Cas9 (trs-cas9, black bar) with putative targeting determinants (green bars). Promoters are shown with arrows indicating the transcriptional start sites. Below, intergenic distances were plotted for $\operatorname{tr}_{\mathrm{L}}^{+}$and $\operatorname{tr}_{\mathrm{L}}^{-}$loci.

Figure 8. Model of $\operatorname{tr}_{L}$ regulation. A) $\operatorname{tr}_{L}$ forms a natural single guide RNA that preserves the structural elements of the tracr:crRNA duplex and artificial sgRNAs. B) In wild-type cells, trL represses $P_{\text {cas }}$ resulting in low levels of Cas gene expression. Low Cas9 levels destabilize tracrRNAs and lead to low levels of tracrRNA:crRNA processing. In this state, the CRISPR-Cas system can interfere against viruses at low MOls but cannot effectively acquire new spacers. crRNAs partially relieve repression by binding to $\operatorname{tr}_{L}$ and preventing formation of the natural single guide. $C$ ) In the absence of $\operatorname{tr}_{L}$, Cas gene expression is induced, $\operatorname{tr}_{S}$ is stabilized by Cas 9 and tracrRNA:crRNA processing is enhanced. As a result, interference can occur at higher MOIs and spacer acquisition is stimulated.

Figure S1. Overview of CRISPR biogenesis. The CRISPR array is transcribed as one long precursor CRISPR RNA (pre-crRNA). tracrRNAs are transcribed from one of two promoters, producing a long-form ( $\left.t r_{L}\right)$ and short-form ( $\left.\operatorname{tr}_{S}\right)$. Once bound to Cas9, $\operatorname{tr}_{S}$ or $\operatorname{tr}_{L}$ can recruit the pre-crRNA through base-pairing between the pre-crRNA repeats (black lines) and a complementary region within $\operatorname{tr}_{S} / \operatorname{tr}_{L}$ (black line). RNAselll, a bacterial enzyme that cleaves dsRNAs, cuts the tracrRNA:pre-crRNA duplex in the middle of the repeat (1 $1^{\circ}$ processing, Fig. S6), releasing RNAselll and the cleaved $5^{\prime}$ end of $\operatorname{tr}_{S} / \operatorname{tr}_{L}$. Following cleavage between all crRNA repeats, Cas9 and the processed trRNA (trp) remain bound to a pre-crRNA fragment containing a single 30nt spacer with half repeats on either side. Next, a second processing event digests the $5^{\prime}$ end of the pre-crRNA fragment leaving a 20 nt spacer $\left(2^{\circ}\right.$ processing) and producing the mature targeting complex (Cas9:trp:crRNA).

Figure S2. Characterization of tracrRNA mutants. A) An extended view of Tn-seq reads in the CRISPR-Cas region for representative - phage (top panel) and + phage (bottom panel) experiments. The location of each hit corresponds to the gene annotations shown in cartoon format below the Tn-seq reads. B-C) Representative plates are shown from the top agar immunity assays performed in Fig. 1D-E. D) Schematic of the tracrRNA promoter region. The wild-type sequence is shown in the top row with transcribed sequences shown in orange. $P_{\operatorname{trL}}$ and $P_{t r s}$ are each indicated with a transcriptional start site $(+1)$ and the -10 and -35 promoter elements that contact RNA polymerase. $\operatorname{In} \operatorname{tr}_{\mathrm{L}}{ }^{*}$ (middle row), two $\mathrm{T}>\mathrm{C}$ mutations in the $\operatorname{tr}_{\mathrm{S}}-10$ element were introduced to prevent trs transcription. In $\Delta \operatorname{tr}_{\mathrm{L}}$ (bottom row), the $\operatorname{tr}$ ( promoter and 19 5' nucleotides have been deleted. E) Infrared northern blot on cells harboring a plasmid 
expressing a naïve wild-type or $\operatorname{tr}_{\mathrm{L}}{ }^{*}$ CRISPR-Cas system probed with oligos matching the $3^{\prime}$ end of tracrRNA. In $\operatorname{tr}_{\mathrm{L}}{ }^{*}, \operatorname{tr}_{\mathrm{S}}$ transcription is below the limit of detection and $\operatorname{tr}_{\mathrm{P}}$ levels are greatly reduced, likely owing to poor processing of $\operatorname{tr}_{L}$ compared to $\operatorname{tr}_{S}$.

Figure S3. $\Delta \operatorname{tr}_{L}$ cells exhibit enhanced CRISPR adaptation and interference. A) Schematic of spacer acquisition assay on cells harboring a plasmid expressing tracrRNA and cas9 and a second plasmid expressing the adaptation-specific Cas genes and CRISPR repeat from the anhydrotetracycline(ATc)-inducible promoter $P_{x y l} /$ tet. ATc was added at $0.5 \mathrm{ug} / \mathrm{mL}$ for 2 hours to induce expression of the adaptation cassette, and spacer acquisition was monitored by an "enrichment PCR" assay. PCR products were run on a $2 \%$ agarose gel, and the presence of a larger band represents a single newly acquired spacer. B-C) Interference assays on cells harboring CRISPR systems with the $\phi N M 4 \gamma 4$-targeting spacer NM2 on medium (B) and low-copy (C) plasmids. Cells were treated with $\phi N M 4 \gamma 4$ at the indicated MOls and cell densities $\left(\mathrm{OD}_{600}\right)$ were measured every 10 minutes in a 96-well plate reader.

Figure S4. Transcriptomic changes in $\Delta \operatorname{tr}_{\mathrm{L}}$. A) RNAseq data from cells harboring a $\Delta \operatorname{tr}_{\mathrm{L}} \mathrm{CRISPR}$ system outgrown from stationary to mid-logarithmic phase for 4 hours. A window is shown focused on the $\mathrm{P}_{\text {cas }}$ region. $\left.B\right)$ RNAseq from cells with a wild-type or $\Delta \operatorname{tr}_{L}$ CRISPR system grown to mid-log phase as in (A) showing sequencing reads across the broader CRISPR-Cas region. Note the $y$-axis is scaled differently in the wild-type and $\Delta \operatorname{tr}_{L}$ experiments. C) Infrared Northern blot on strains from (B) grown overnight to late stationary phase ("stat") or diluted back from stationary into logarithmic phase for 4 hours ("log"). Membranes were probed with oligos matching the 3' end of tracrRNA (top panel) or the 4.5S RNA (middle panel). The bottom panel shows a Western blot (WB) for Cas9.

Figure S5. Extended characterization of the determinants of $\operatorname{tr}_{L}: P_{\text {cas }}$ repression. A) Basepairing between the $5^{\prime}$ end of $\operatorname{tr}_{L}$ and the $P_{\text {cas }}$ region just downstream from the transcriptional start site (+1). -10 and +1 promoter elements are shown in green, and the PAM downstream

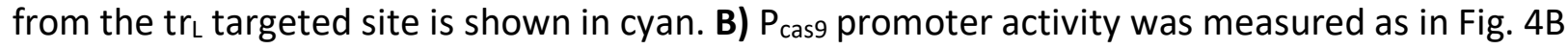
with the absolute values for both +/- Cas9+tracrRNA(tr) conditions shown. C) Promoter activity

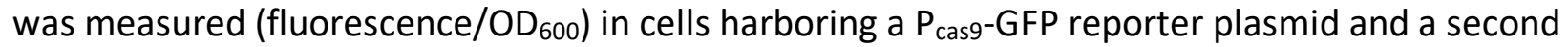
plasmid expressing wild-type tracrRNA or $\Delta \operatorname{tr}_{L}$ and wild-type cas9 or a cas 9 null mutant in which two stop codons were inserted after the $15^{\text {th }}$ codon. D) Infrared Northern blot on the strains in (C) grown to stationary phase. Membranes were probed with oligos matching the $3^{\prime}$ end of tracrRNA. E) Promoter activity was measured (fluorescence/OD 600 ) in cells harboring a plasmid expressing cas9 from a constitutive promoter and $\mathrm{P}_{\text {cas9-GFP }}$ and a second plasmid expressing the indicated tracrRNA mutants. The ratio of activities for the indicated tracrRNA mutants relative to $\Delta \operatorname{tr}_{\mathrm{L}}$ are shown. Numbers indicate the positions of nucleotides mutated to their complementary base relative to the $5^{\prime}$ end of $\operatorname{tr}_{L}$; $\Delta$ upper stem extension; nucleotides $26-104$ of $\operatorname{tr}_{L}$ were replaced by a 5'-GAAA-3' tetraloop. F) Schematic showing the base-pairing potential of the $\operatorname{tr}_{\mathrm{L}}$ mutants from (E). 
Figure S6. Structures of CRISPR-Cas RNAs. Schematics are shown for the putative tr $\mathrm{L}$ natural sgRNA (A), the trs:pre-crRNA duplex (B), the processed trp:crRNA duplex (C), the artificial sgRNA commonly used in CRISPR editing technologies (D), and the $\operatorname{tr}_{L}$ :pre-crRNA duplex (E). Green, lower stem; dark yellow, bulge; cyan, upper stem; pink, nexus; purple, termination hairpins; brown, region of crRNA:tracrRNA complementarity outside the stems; gray, putative structures of $\operatorname{tr}$ regions of unknown function.

Figure S7. Extended $\operatorname{tr}_{\mathrm{L}}$ match lengths lead to tighter repression and cleavage. A) Promoter activity was measured (fluorescence/OD 600 ) in cells harboring variants of pRW22 (Fig. 5A) with target site matches of the indicated lengths and a second GFP reporter plasmid, pJW711 or pCN57. Grey bars in the schematic, targeting site; EV, empty vector; WT, wild-type tr targeting $P_{\text {cas9. }}$ B) Cells harboring pRW22 variants with the indicated target site match lengths were transformed with a second plasmid, EV, pJW711 or pCN57. Transformants were plated on selective media with antibiotics to maintain both plasmids, and surviving colonies were counted. Data is presented as a ratio of pJW711 or pCN57 colonies relative to the EV control.

Figure S8. crRNAs interact with tr $r_{L}$ to regulate CRISPR-Cas levels and activity. A-B) Infrared Northern blot of $(A)$ strains from Fig. 6A or (B) cells harboring a plasmid with a naïve wild-type or $\Delta \operatorname{tr}_{L}$ CRISPR system and a second empty vector or a plasmid constitutively expressing cas 9 from $P_{\text {spac }}$ (+cas9). Membranes were probed with oligos matching the 3 ' end of tracrRNA (top), the crRNA repeat (middle) or the 4.5S RNA (bottom, loading control). Bottom panels, Western blot (WB) using an $\alpha$-Cas9 antibody. Membranes were stained with Ponceau $S$, and the prominent band is shown as a loading control (total protein, TPN). C) Top agar interference assay with cells harboring a plasmid expressing Cas9, the indicated tracrRNA mutants and the $\phi N M 4 \gamma 4$-targeting spacer NM2. 10-fold dilutions of $\phi N M 4 \gamma 4$ were plated on the indicated bacterial lawns. $\Delta$ tr, deletion of the tracrRNA locus; wt, wild-type tracrRNA; $\Delta \operatorname{tr}_{L}$, deletion of the long-form tracrRNA; $\operatorname{tr}_{\mathrm{L}}{ }^{*}, \mathrm{P}_{\text {trs }}$ mutant expressing long-form tracrRNA only; $\mathrm{P}_{\text {cas }}{ }^{\mathrm{NGC}}, \mathrm{P}_{\text {cas }} \mathrm{PAM}$ mutant that is insensitive to $\operatorname{tr}_{\mathrm{L}}$ repression. D) Interference assay with $\phi N M 4 \gamma 4$ at $\mathrm{MOI}=10$ with the indicated strains from Figure 6B. IPTG was added at $1 \mathrm{mM}$ to induce expression from $\mathrm{P}_{\text {sparc2 }}$. E) Infrared Northern blot on cells harboring a plasmid expressing cas 9 and the indicated tracrRNA mutants from the $\mathrm{P}_{\text {cas }}{ }^{\mathrm{NGC}}$ promoter and a second plasmid expressing additional $\operatorname{tr}_{L}$ or an empty vector. Membranes were probed with oligos matching the $3^{\prime}$ end of $\operatorname{tracrRNA.~} \operatorname{tr}_{L}{ }^{*}(-$ )/ $/ r_{L}{ }^{*}(+)$, cells harboring plasmids expressing $\operatorname{tr}_{\mathrm{L}}{ }^{*}$ from the $\mathrm{P}_{\text {sparc2 }}$ IPTG-inducible promoter were treated without or with IPTG for 1:15 hours respectively. F) Quantification of Western blot experiments described in Figure 6C, performed in biological triplicate.

Figure S9. Sequence alignment of tr targeting determinants. A MAFFT multiple sequence alignment was constructed of the candidate targeting sequences (CTS) and lower stems (LS) from all $\operatorname{tr}_{\mathrm{L}}{ }^{+}$genomes. The phylogenetic tree was constructed as for Figure 7 using Cas 9 protein sequences.

Figure S10. Sequence alignment of full-length $\operatorname{tr}_{L}$. A MAFFT multiple sequence alignment was constructed of $\operatorname{tr}_{\mathrm{L}}$ from all $\mathrm{tr}_{\mathrm{L}}^{+}$genomes. Orange, candidate targeting sequence (CTS); green, lower stem (LS); blue, upper stem (US); black line, upper stem extension (USE); purple, nexus. 
Figure S11. Evidence of variation and degeneration within Pcas9 and its targeting determinants. A) A MAFFT multiple sequence alignment was constructed for the $P_{\text {cas }}$ regions of $\mathrm{tr}_{\mathrm{L}}{ }^{+}$genomes. Yellow boxes, predicted -35 and $-10 \mathrm{P}_{\text {cas } 9}$ promoter elements; orange underline, $\mathrm{P}_{\text {cas }}$ target site; light blue boxes, PAM. B-C) A multiple sequence alignment spanning the $\operatorname{tr}_{L}$ candidate targeting sequence (CTS, orange underline) and lower stem (LS, grey underline) through the $\mathrm{P}_{\text {cas }}$ target site in several S. pseudopneumoniae strains (B) and a $S$. pyogenes local branch (C). Yellow and black boxes, putative -10 and +1 promoter elements respectively. In S. equi zooepidemicus, the targeting determinants contain seed mismatches (blue underline) but retain an LS and PAM.

Supplemental Table 1. An Excel file containing NGS data from the experiments detailed in Figure 1. Separate worksheets are provided showing NGS reads that mapped to the genome and CRISPR plasmid separately. The -CRISPR library was treated without phage in single and with phage in duplicate. The +CRISPR library was treated without phage in single and with phage in triplicate.

Supplemental Table 2. An Excel file containing metadata for the bioinformatic analysis described in Figure 7.

Supplemental Table 3. An Excel file containing RNA-seq data for the experiments described in Fig. S4.

Supplemental Materials. An Excel file with three worksheets detailing the strains, oligos and plasmids used in this paper. Plasmid construction details are also provided. 


\section{METHODS}

\section{Growth conditions}

Staphylococcus aureus strains were grown at $37^{\circ} \mathrm{C}$, unless otherwise indicated, in Bacto Brain-Heart infusion (BHI) broth with shaking at 220 RPM. During outgrowths from stationary phase preceding phage treatments, $\mathrm{BHI}$ was supplemented with calcium chloride at $5 \mathrm{mM}$ to allow phage adsorption and with 1 mM IPTG to allow expression from $\mathrm{P}_{\text {sparc2 }}$ when necessary. Antibiotics were used at the following concentrations for strain construction and plasmid maintenance in S. aureus: tetracycline, $5 \mu \mathrm{g} / \mathrm{mL}$; chloramphenicol, $10 \mu \mathrm{g} / \mathrm{mL}$; erythromycin, $10 \mu \mathrm{g} / \mathrm{mL}$; spectinomycin, $250 \mu \mathrm{g} / \mathrm{mL}$.

\section{Strain and plasmid construction}

See Supplemental Materials for strains, plasmids, cloning notes and oligos used in this study.

\section{Gibson assembly}

Gibson assemblies were performed as described ${ }^{1}$. Briefly, $100 \mathrm{ng}$ of the largest dsDNA fragment to be assembled was combined with equimolar volumes of the smaller fragment(s) and brought to $5 \mu \mathrm{L}$ total in $\mathrm{dH}_{2} \mathrm{O}$ on ice. Samples were added to $15 \mu \mathrm{L}$ of Gibson Assembly master mix, mixed by pipetting and incubated at $50^{\circ} \mathrm{C}$ for 1 hour. Samples were drop dialyzed in $\mathrm{dH}_{2} \mathrm{O}$ for 30 minutes -1 hour, and $5 \mu \mathrm{L}$ were electroporated into $50 \mu \mathrm{L}$ electrocompetent RN4220 S. aureus cells.

\section{Oligo cloning}

To create a repeat-spacer-repeat CRISPR array with a defined spacer, we used a restriction digest-based cloning approach. Parent plasmids contain two CRISPR repeats flanking a 30bp sequence housing two Bsal restriction sites. 400-800 ng of the plasmid was mixed with the Bsal-HFv2 restriction enzyme (NEB, R3733S) in a $10 \mu \mathrm{L}$ reaction volume ( $1 \mu \mathrm{L}$ Bsal-HFv2 enzyme, $1 \mu \mathrm{L}$ CutSmart buffer, plasmid + nucleasefree water to $10 \mu \mathrm{L}$ ) and incubated at $37^{\circ} \mathrm{C}$ for 8 hours. Two IDT oligos, a "top" strand with sequence $5^{\prime}$ AAAC-(30bp spacer)-G-3', and a "bottom" strand with sequence 5'-AAAAC-(30bp spacer reverse complement)-3' were phosphorylated with PNK (NEB, M0201S) in a $50 \mu \mathrm{L}$ reaction volume $(1.5 \mu \mathrm{L} 100$ uM top oligo, $1.5 \mu \mathrm{L} 100 \mathrm{uM}$ bottom oligo, $41 \mu \mathrm{L}$ nuclease free water, $5 \mu \mathrm{L}$ T4 PNK $5 x$ reaction buffer, 1 $\mu \mathrm{LT} 4 \mathrm{PNK}$ ) at $37^{\circ} \mathrm{C}$ for 30 minutes $-1 \mathrm{hr}$. After phosphorylation, oligos were annealed by adding $2.5 \mu \mathrm{L}$ of $1 \mathrm{M} \mathrm{NaCl}$ to the $50 \mu \mathrm{L}$ reaction and incubating for 5 minutes at $98^{\circ} \mathrm{C}$, then allowing the reaction to cool to room temperature (1-2 hours). The phosphorylated, annealed oligos were diluted 1:10 in nuclease-free water and ligated to the digested plasmid in a $20 \mu \mathrm{L}$ reaction (10 $\mu \mathrm{L}$ digested plasmid, $6 \mu \mathrm{L}$ water, $1 \mu \mathrm{L}$ 1:10 diluted oligos, $2 \mu \mathrm{L} \mathrm{T4}$ ligase buffer, $1 \mu \mathrm{L}$ T4 DNA ligase enzyme (NEB, M0202S)) at room temperature overnight. Reactions were drop dialyzed for 1 hour in $\mathrm{dH}_{2} \mathrm{O}$ and $5 \mu \mathrm{L}$ were transformed into electrocompetent RN4220 S. aureus cells.

\section{Tn-seq screen}

To construct a transposon library in cells lacking a CRISPR system, Staphylococcus aureus RN4220 cells harboring pTV1, a temperature-sensitive plasmid containing transposon Tn917 from Streptococcus faecalis ${ }^{2}$, were grown overnight at the permissive temperature $\left(30^{\circ} \mathrm{C}\right)$ in $\mathrm{BHI}$ supplemented with chloramphenicol. The next morning, $500 \mu \mathrm{L}$ cells were washed $1 \mathrm{x}$ in plain $\mathrm{BHI}$ and then diluted into 500 
$\mathrm{mL} \mathrm{BHI} \mathrm{prewarmed} \mathrm{to} \mathrm{the} \mathrm{restrictive} \mathrm{temperature}\left(42^{\circ} \mathrm{C}\right)$ and supplemented with erythromycin, without chloramphenicol. Cells were grown at $42^{\circ} \mathrm{C}$ with shaking for $\sim 8$ hours and diluted again $500 \mu \mathrm{L}$ into 500 $\mathrm{mL} \mathrm{BHI} /$ erythromycin. After an overnight growth at $42^{\circ} \mathrm{C}$, cells were plated onto $\mathrm{BHI}$ agar plates supplemented with erythromycin and incubated overnight at $42^{\circ} \mathrm{C}$. The next day, 10,000 colonies were scraped with $3 \mathrm{~mL} \mathrm{BHI}$ into a Falcon tube and resuspended by pipetting and vortexing. DMSO was added to $10 \%$ and library aliquots were stored at $-80^{\circ} \mathrm{C}$. A second transposon library was constructed in cells harboring the CRISPR-Cas system on plasmid pJW92 as above, with tetracycline added during each step for plasmid maintenance.

Transposon library aliquots were thawed and diluted to $\mathrm{OD}=0.05$ in $20 \mathrm{~mL}$ (- phage experiments) or 500 $\mathrm{mL}$ (+ phage experiments) BHI supplemented with calcium chloride. After $\sim 2$ hours of growth at $37^{\circ} \mathrm{C}$, culture ODs were roughly 0.4-0.5, and $\phi N M 4 \gamma 4$ was added to the + phage experiments in duplicate (CRISPR- library) or triplicate (CRISPR+ library) at $\mathrm{MOI}=1$. After 24 hours of growth, genomic DNA was prepared using the Wizard Genomic DNA purification kit (Promega) and culture aliquots were frozen at $80^{\circ} \mathrm{C}$ for storage in $10 \%$ DMSO. As above, tetracycline was added in all steps for cells harboring pJW92.

To prepare NGS libraries, genomic DNA was digested with NEBNext dsDNA Fragmentase (M0348) for 9 minutes, and the reaction was stopped by addition of $0.1 \mathrm{M}$ EDTA (final concentration). The fragmented DNA, centered at 700bp, was then purified using the QIAquick PCR Purification kit and end repaired using the NEBNext DNA Library Reagent Set for Illumina (E6000S). After another QIAquick PCR purification, NEBNext adapters were ligated onto the fragments and DNA in the $0.2-1 \mathrm{~kb}$ range was purified from an agarose gel with the QIAquick Gel Extraction kit. Next, for each sample, a PCR was performed with a unique oligo from the NEBNext Multiplex Oligos for Illumina kit (E7335S, Index Primers Set 1) and a universal, transposon-specific primer (oJW451). Amplicons in the $0.2-0.5 \mathrm{~kb}$ range were gel purified as before and sequenced on an Illumina HiSeq using the sequencing primer oJW436. Sequencing reads were processed with custom Python scripts and aligned to the NCTC8325 genome using the Burrows-Wheeler Aligner. Tn-seq output was visualized with the Integrative Genomics Viewer ${ }^{3}$.

\section{S. aureus miniprep protocol}

1-1.5 $\mathrm{mL}$ of an overnight culture, unless otherwise indicated, was pelleted and resuspended in $250 \mu \mathrm{L}$ Buffer P1. 10-20 $\mu$ L lysostaphin (Ambi Products LLC, LSPN-50, $100 \mu \mathrm{g} / \mathrm{mL}$ final) was added and the cells were incubated without shaking at $37^{\circ} \mathrm{C}$ for $\sim 20$ minutes. Following lysis, plasmids were isolated using the QIAGEN Spin Miniprep kit according to the manufacturer's protocol, beginning with addition of P2. DNA was eluted from each column in $30 \mu \mathrm{L}$ RNAse-free water.

\section{Electroporation adaptation assay}

Electrocompetent $S$. aureus cells were made by washing overnight cultures twice in full-volumes of $\mathrm{dH}_{2} \mathrm{O}$ and once in a half-volume of $\mathrm{dH}_{2} \mathrm{O}$ before resuspending in 1/100 the original volume in $10 \%$ glycerol. 50 ng of dialyzed DNA, amplified from phage $\phi N M 4 \gamma 4$ with primers JW1744 and JW1745, were added to 50 $\mu \mathrm{L}$ of electrocompetent cells in an Eppendorf tube, mixed by pipetting and left at RT for 10 minutes. Cells were transferred to a $0.2 \mathrm{~cm}$ electroporation cuvette (Bio-Rad, 1652086) and electroporated at $1.8 \mathrm{kV}$ using an Eporator (Eppendorf). Following electroporation, $1 \mathrm{~mL} \mathrm{BHI}$ broth was added and the contents of the cuvette were mixed by pipetting and then transferred to an Eppendorf tube and grown for 1 hour with shaking at $220 \mathrm{RPM}$ at $37^{\circ} \mathrm{C}$. Plasmids were miniprepped, and spacer acquisition was monitored by a 
spacer-specific PCR reaction with primers oJW1833 and oJW1834. PCR products were visualized on a $2 \%$ agarose gel run for 25 minutes at $130 \mathrm{~V}$.

\section{Overexpression adaptation assay}

Overnight cultures of $\mathrm{S}$. aureus were diluted to $\mathrm{OD}=0.04$ in $\mathrm{BHI}$ broth supplemented with antibiotics and grown for 1 hour with shaking at $37^{\circ} \mathrm{C}$. Cultures were then supplemented with anhydrotetracycline (ATc) at $0 \mu \mathrm{g} / \mathrm{mL}$ or $0.5 \mu \mathrm{g} / \mathrm{mL}$ (final concentrations). Cultures were incubated with shaking at $37^{\circ} \mathrm{C}$ for 2 hours and then miniprepped with a lysis step including $15 \mu \mathrm{L}$ of lysostaphin incubated at $37^{\circ} \mathrm{C}$ for 5 minutes. An enrichment PCR was performed as described ${ }^{4,5}$ and PCR products were visualized on a $2 \%$ agarose gel run for 27 minutes at $130 \mathrm{~V}$.

\section{Liquid growth interference assay}

Overnight cultures of $S$. aureus were diluted to $\mathrm{OD}=0.025$ in $\mathrm{BHI}$ broth supplemented with calcium chloride and antibiotics and grown for 1 hour and 15 minutes shaking at $37^{\circ} \mathrm{C}$. Cultures were normalized to the OD of the lowest culture and phage $\phi N M 4 \gamma 4$ was added to the appropriate $\mathrm{MOI}$. After inverting to mix, $150 \mu \mathrm{L}$ of each culture were added to a flat-bottom 96-well plate (Grenier 655180), and the plate was incubated at $37^{\circ} \mathrm{C}$ with shaking in a TECAN Infinite $\mathrm{F}$ Nano+ with $\mathrm{OD}_{600}$ measurements recorded every 10 minutes for 24 hours.

\section{Top agar interference assay}

$100 \mu \mathrm{L}$ of $\mathrm{S}$. aureus overnight cultures were added to a $50 \mathrm{~mL}$ Falcon tube. $6 \mathrm{~mL}$ of BHI top agar $(0.75 \%$ agar) supplemented with calcium chloride $(5 \mathrm{mM}$ final concentration) were added to each tube. After swirling to mix, the cells and top agar were poured onto a BHI 1.5\% agar plate and rocked gently to create a bacterial lawn. The plate was incubated for 15 - 30 minutes at RT. $3.5 \mu \mathrm{L}$ of 810 -fold serial dilutions of phage $\phi N M 4 \gamma 4$ in $\mathrm{BHI}$ broth were spotted on top of the bacterial lawn using a multichannel pipette. After a 30 minute incubation at $\mathrm{RT}$, the plates were moved to a $37^{\circ} \mathrm{C}$ incubator overnight.

\section{Promoter activity fluorescence assay}

$200 \mu \mathrm{L}$ of each overnight culture was spun down in a $1.5 \mathrm{~mL}$ Eppendorf tube at 6,000 RPM for one minute. Cell pellets were resuspended in $1 \mathrm{~mL}$ of $1 \mathrm{X}$ PBS and $150 \mu \mathrm{L}$ were transferred into a clear, flatbottomed 96-well plate (Grenier 655180). Measurements for absorbance (at $600 \mathrm{~nm}$ ) and fluorescence (excitation wavelength $=485 \mathrm{~nm}$; emission wavelength $=535 \mathrm{~nm}$ ) were taken using a TECAN Infinite $F$ Nano+. For each experimental strain, promoter activity was measured as $(\mathrm{Fe}) /(\mathrm{Ae})-(\mathrm{Fc}) /(\mathrm{Ac})$ where $\mathrm{F}=$ fluorescence, $\mathrm{A}=$ absorbance, $\mathrm{e}=$ experimental strain and $\mathrm{c}=$ non-fluorescent control strain.

\section{Liquid growth immunity assay}

Overnight cultures of Staphylococcus aureus harboring naïve CRISPR systems with a single repeat were diluted to $\mathrm{OD}=0.1$ in $\mathrm{BHI}$ supplemented with calcium chloride and relevant antibiotics and grown for 1.5 hours. Cultures were normalized to the OD of the lowest culture, and phage $\phi N M 4 \gamma 4$ was added at the specified MOIs. $150 \mu \mathrm{L}$ of each culture was added to a clear, flat-bottom 96-well plate (Grenier 655180), and the plate was incubated at $37^{\circ} \mathrm{C}$ with shaking in a TECAN Infinite $\mathrm{F} \mathrm{Nano+} . \mathrm{OD}_{600}$ measurements were recorded every 10 minutes for 24 hours. 


\section{Top agar immunity assay}

$100 \mu \mathrm{L}$ of overnight cultures of Staphylococcus aureus harboring naïve CRISPR systems were mixed with $\phi N M 4 \gamma 4$ at $\mathrm{MOI}=25$ and $6 \mathrm{~mL}$ of $\mathrm{BHI}$ top agar (0.75\% agar) supplemented with calcium chloride and poured onto $\mathrm{BHI}$ agar (1.5\%) plates. After 15 minutes at RT, plates were moved to a $37^{\circ} \mathrm{C}$ incubator overnight, and surviving colonies were counted. When indicated, colony PCR was performed with primers oJW154 and oJW355 to verify expansion of the CRISPR array due to spacer integration.

\section{PCR conditions}

PCR was performed with Phusion HF DNA polymerase using 5X Phusion Green Reaction Buffer (Thermo). Each reaction contained $10 \mu \mathrm{L}$ buffer, $4 \mu \mathrm{L}$ dNTPs, $0.5 \mu \mathrm{L}$ each of $100 \mu \mathrm{M}$ forward and reverse primers, 10-50 ng template, $0.5 \mu \mathrm{L}$ polymerase and nuclease-free water to $50 \mu \mathrm{L}$. Three-step cycling was performed under the following conditions: $98^{\circ} \mathrm{C}$ for 30 seconds, 34 cycles of $\left[98^{\circ} \mathrm{C} 5\right.$ seconds, $45-72^{\circ} \mathrm{C} 15$ seconds, $72^{\circ} \mathrm{C}$ for $\left.30 \mathrm{~s} / \mathrm{kb}\right], 72^{\circ} \mathrm{C} 10$ minutes, hold at $10^{\circ} \mathrm{C}$.

\section{RNA extraction}

To extract RNA for Northern blot, RT-qPCR, and RNA-seq analysis, 7.5E8 cells from an overnight culture were spun down, resuspended in $150 \mu \mathrm{L}$ 1X PBS (10X stock, Corning, 46-013-CM) and lysostaphin (60 $\mu \mathrm{g} / \mathrm{ml}$ final concentration), and incubated at $37^{\circ} \mathrm{C}$ for 5 minutes. To the whole cell lysate, $450 \mu \mathrm{L}$ Trizol (Zymo, R2071) and $600 \mu \mathrm{L} 200$ proof ethanol were added, samples were vortexed and RNA was extracted using the Direct-Zol Miniprep Plus spin column according to the manufacturer's protocol (Zymo, R2071). Samples were eluted in $50 \mu \mathrm{L}$ Ambion RNAse-free water (ThermoFisher, AM9937).

\section{Infrared Northern (irNorthern)}

Total RNA (3-10 $\mu$ g) was mixed 1:1 with 2X Novex sample buffer (Thermo, LC6876), boiled at $94^{\circ} \mathrm{C}$ for 3 minutes and placed on ice for 3-5 minutes. Samples were loaded onto a 15\% TBE-Urea gel (MINI-Protean, Bio-rad, 4566053) and run at $150 \mathrm{~V}$ for 2-2.5 hours. A Hybond N+ membrane (GE lifesciences, 45000854) and 6 sheets of $3 \mathrm{~mm}$ Whatman cellulose paper (Sigma Aldrich, WHA3030861) were pre-soaked for 5 minutes in room temperature 0.5X TBE, then assembled into a sandwich: 3 layers Whatman paper, Hybond membrane, TBE-Urea gel, and 3 more layers of Whatman paper. Blotting was performed using a Trans-blot Turbo (Bio-Rad) at $200 \mathrm{~mA}$ for 30 minutes. The membrane was then pre-hybridized in $10 \mathrm{~mL}$ ExpressHyb (Clontech, NC9747391) at $44^{\circ} \mathrm{C}$ in a rotating oven for 1 hour, and probed overnight at $44^{\circ} \mathrm{C}$, with rotation, using probes conjugated to irDyes (LICOR; 680CW 929-50010, 800CW 929-50000), made following the protocol detailed at (https://bio-protocol.org/e3219) ${ }^{6}$. The membrane was washed once with 2 XSC/0.1\% SDS, and once with 1 XSC/0.1\% SDS each for 10 minutes at RT, then visualized on the Odyssey Fc (LICOR). 4.5S RNA was used as a loading control (stability of $4.5 \mathrm{~S}$ across genetic backgrounds was verified by qPCR). Sequences for oligos used to probe crRNA, tracrRNA and 4.5S RNA (oJW2313, oJW1991, oJW2172) are listed in Supplementary Materials.

\section{Western blot}

$1.8 \mathrm{E} 8$ cells were resuspended in $1 \mathrm{X}$ PBS supplemented with lysostaphin ( $60 \mu \mathrm{g} / \mathrm{ml}$ final concentration) and incubated at $37^{\circ} \mathrm{C}$ for 20 minutes. Whole cell lysate was mixed 1:1 with $2 x$ Laemmli solution (Bio-rad, 
1610737) supplemented with B-mercaptoethanol (55 mM stock, ThermoFisher, 21985023) at a final concentration of $1.3 \mathrm{mM}$, and boiled at $98^{\circ} \mathrm{C}$ for 10 minutes. Samples were loaded onto a 4-20\% Trisglycine gel (MINI-Protean TGX Pre-cast, Bio-rad, 4561095) and run at $200 \mathrm{~V}$ for 15 min-1 hour. A PVDF membrane (Bio-rad) was hydrated with methanol for 15-30 seconds and pre-wet alongside a stack of blotting paper for 3-5 minutes in 1X Transfer buffer. A blotting sandwich was assembled consisting of six layers (one stack) of filter paper, the PVDF membrane, Tris-glycine gel, and another stack of filter paper. Samples were transferred onto a nitrocellulose membrane with the Trans-blot turbo (Bio-Rad, 1704150), with high molecular weight transfer settings (1.6 A for 10 minutes), and the membrane was stained with Ponceau for 5 minutes to perform total protein normalization. The membrane was blocked with $5 \%$ nonfat dry milk for 1 hour, then probed with a 1:1000 dilution of Cas9 monoclonal antibody (Cell Signaling, 7A9-3A3) for 2 hours at RT, or at $4^{\circ} \mathrm{C}$ overnight. The membrane was washed with 1 X TBST buffer $3 x$ for 10 minutes at RT, then probed with 1:15,000 dilution of infrared (LICOR, 925-32210) or 1:10,000 dilution of HRP (Pierce, PA174421) secondary antibody for 1 hour at room temperature. The membrane was washed as before, then visualized on the Odyssey Fc (LICOR).

\section{RNA-seq library preparation and sequencing}

To prepare libraries for next-generation sequencing, we first depleted ribosomal RNA from the total purified RNA using reverse capture of biotinylated probes annealing to rRNA with streptavidin beads, following the protocol detailed at (https://mbio.asm.org/content/11/2/e00010-20) ${ }^{7}$. rRNA depleted samples were prepared for sequencing using the Cleantag Small RNA library prep kit (Trilink, L-3206-24) according to the manufacturer's instructions, with 10 ng RNA input and 18 PCR cycles. Sequencing was performed on an Illumina MiSeq with a v3 2x75 cycle kit at the Johns Hopkins Genome Resources Core Facility (GRCF). Full data is provided in Supplemental Table 3.

\section{qPCR}

Purified total RNA (1-4 $\mu \mathrm{g}$ ) was treated with TURBO DNA-free (ThermoFisher, AM1907) and reverse transcribed with Superscript IV (ThermoFisher, 18090050) according to the manufacturer's instructions. cDNA was diluted to 0.5-1 ng/ $\mu \mathrm{L}$ and 4-8 $\mathrm{ng}$ was used as input in an $8 \mu \mathrm{L}$ volume. $1 \mu \mathrm{L}$ of $10 \mu \mathrm{M}$ forward/reverse primers were used, and $10 \mu \mathrm{L}$ of $2 X$ PowerUp SYBR mastermix (ThermoFisher, A25742). qPCR was performed with cycling conditions: $50^{\circ} \mathrm{C}$ for 2 minutes, $95^{\circ} \mathrm{C}$ for 2 minutes, 39 cycles of $\left[60^{\circ} \mathrm{C} 1\right.$ minute], followed by a melt curve: $65^{\circ} \mathrm{C}$ to $95^{\circ} \mathrm{C}$, incrementing $0.5^{\circ} \mathrm{C}$ every 5 seconds. rho was used as a loading control and was amplified using primers oJW2003/2004. cas9 was amplified with primers oW262/oJW1986. cas1 was amplified using primers oJW150/oL432. csn2 was amplified using primers oJW2139/2140. The region spanning csn2 and crRNA (csn2_cr) was amplified using oJW2141/2142. The pre-crRNA was amplified using oJW2142/2143. All forms of tracrRNA were amplified using oJW2012/2013. qPCR primer sequences are provided in Supplementary Materials.

\section{Transformation assay}

$10 \mathrm{~mL}$ overnight cultures of cells harboring derivatives of pTP16, which encodes cas 9 and tracrRNA variants with increasing match lengths to the GFP reporter plasmids pJW711 and pCN57 were diluted to $\mathrm{OD}=0.1$ and outgrown in $\mathrm{BHI}$ supplemented with chloramphenicol for 1-2 hours until the OD was between 0.8-1. The cultures were centrifuged at 4200 RPM for 10 minutes and washed twice with $1 \mathrm{~mL}$ of ice-cold deionized water in a $1.5 \mathrm{~mL}$ Eppendorf tube with 6000 RPM 1 minute spins between washes. Cells were resuspended in $150 \mu \mathrm{L} 10 \%$ glycerol and stored at $-80^{\circ} \mathrm{C}$ if not used immediately. $50 \mathrm{ng}$ of 
pJW711, pCN57, or an empty vector pE194 was electroporated into $50 \mu \mathrm{L}$ of competent cells, and outgrown in $300 \mu \mathrm{L} \mathrm{BHI}$ at $37^{\circ} \mathrm{C}$ with shaking for 3 hours. $100 \mu \mathrm{L}$ of each outgrowth was plated onto $\mathrm{BHI}$ agar plates supplemented with chloramphenicol and erythromycin and total transformants were counted the following day.

\section{In vitro transcription (IVT)}

IVT templates were generated using the templates pTP16 and pRW22-26 with forward primers oJW22682274 , each of which begin with the T7 promoter sequence (5'- GAAATTAATACGACTCACTATAGG-3'), and the reverse primer oJW2267. To generate the WT long-form tracr, pTP16 was amplified with oJW2267 and oJW2268. To generate the GFP-targeting tracr with an 11-bp match, pRW22 was amplified with oJW2267 and oJW2269. To generate the GFP-targeting tracr with a 13-bp match, pRW23 was amplified with oJW2267 and oJW2270. To generate the GFP-targeting tracr with a 15-bp match, pRW24 was amplified with oJW2267 and oJW2271. To generate the GFP-targeting tracr with a 17-bp match, pRW25 was amplified with oJW2267 and oJW2272. To generate the GFP-targeting tracr with a 19-bp match, pRW26 was amplified with oJW2267 and oJW2273. To generate the sgRNA version of the GFP targeting tracr with a 20-bp match, pRW27 was amplified with oJW2267 and oJW2274. tracrRNA (wild-type and variants) was in-vitro transcribed from $1 \mu \mathrm{g}$ of these templates using the HiScribe T7 High Yield RNA synthesis kit (NEB, E2040S). Reactions were incubated at $37^{\circ} \mathrm{C}$ for 4 hours and run on a $6 \%$ TBE-Urea gel (Invitrogen, EC6265BOX). Bands of the appropriate size were excised, added to a sterile Eppendorf tube in $450 \mu \mathrm{L}$ gel extraction buffer (1X: 0.3 M NaOAc, $10 \mathrm{mM}$ Tris-Cl pH 7.5, $1 \mathrm{mM}$ EDTA), and incubated overnight at $4^{\circ} \mathrm{C}$ in an end-over-end rotator. The buffer with dissolved gel slice was added to $1 \mathrm{~mL}$ icecold $100 \%$ ethanol and $2 \mu \mathrm{L}$ of glycogen $\left(20 \mathrm{mg} / \mathrm{ml}\right.$, ThermoFisher, R0561) and incubated at $-20^{\circ} \mathrm{C}$ for at least one hour. Samples were centrifuged at max speed at $4^{\circ} \mathrm{C}$ for 30 minutes, the supernatant was decanted, and the pellet was washed with $1 \mathrm{~mL}$ cold $70 \%$ ethanol. After another centrifugation step at max speed for 10 minutes at $4^{\circ} \mathrm{C}$, the pellets were either dried in a vacuum centrifuge or let air dry for 10 minutes and eluted in Ambion RNAse-free water.

\section{Electrophoretic mobility shift assay (EMSA)}

DsDNA targets were prepared by mixing $20 \mu \mathrm{L}$ of $20 \mu \mathrm{M}$ top and bottom strand oligos (Pcas: oJW2507/2508, Pgfp:oJW2509/2510) in annealing buffer (10 mM Tris pH 7.5, 50 mM NaCl, 1 mM EDTA), heating to $95^{\circ} \mathrm{C}$ for 5 minutes, then slowly cooling the mixture to room temperature for $1-2$ hours. Annealed oligos were mixed with $4 \mu \mathrm{L} 6 \mathrm{X}$ loading dye (NEB, B7024S), loaded onto a lab-made $8 \%$ TBE gel (Acrylamide/Bis solution 37.5:1, Bio-rad, 1610148), run at 200V for 1 hour, then stained with SYBR Gold (ThermoFisher, S11494) for 10 minutes still, and 10 minutes shaking. Bands of the appropriate size were excised and added to a sterile Eppendorf tube in $450 \mu \mathrm{L}$ gel extraction buffer (1X: $0.3 \mathrm{M} \mathrm{NaOAc}, 10 \mathrm{mM}$ Tris-Cl pH 7.5, 1 mM EDTA), and incubated overnight at room temperature in a shaker. Gel extraction buffer was added to $1 \mathrm{~mL}$ ice-cold $100 \%$ ethanol and $2 \mu \mathrm{L}$ of glycogen $(20 \mathrm{mg} / \mathrm{mL}$, ThermoFisher) and incubated at $-20^{\circ} \mathrm{C}$ for at least one hour. Samples were centrifuged at max speed at $4^{\circ} \mathrm{C}$ for 30 minutes, and the pellet was washed with $1 \mathrm{~mL}$ cold $70 \%$ ethanol. Following another centrifugation step at max speed for 10 minutes at $4^{\circ} \mathrm{C}$, pellets were either dried in a vacuum centrifuge or let air dry for 10 minutes, then eluted in RNAse-free water (Ambion). Purified dsDNAs were diluted to $200 \mathrm{nM}$ with binding buffer (20 mM HEPES pH 7.5, 250 mM KCl, 2 mM MgCl2, 0.01\% Triton X-100, 0.1 mg/mL BSA, 10\% glycerol), then diluted again 1:10 in binding buffer to make a $20 \mathrm{nM}$ stock.

After dsDNA purification, the substrate was radiolabeled with P32 (1 $\mu \mathrm{L} 20 \mathrm{nM}$ oligo duplex, $5 \mu \mathrm{L}$ T4 PNK buffer, $10 \mathrm{mM}$ radioactive ATP, $2 \mu \mathrm{L}$ T4 PNK in a $50 \mu \mathrm{L}$ reaction). This reaction was incubated at $37^{\circ} \mathrm{C}$ for 1 
hour and cleaned up with ProbeQuant G50 spin columns (GE Healthcare, GE28-9034-08) as per manufacturer's instructions.

Wild-type $\operatorname{tr}_{L}$ was prepared by performing in vitro transcription as described in in vitro transcription methods above. $\operatorname{Tr}_{L}$ was diluted to $8 \mu \mathrm{M}$ in RNA hybridization buffer $(20 \mathrm{mM}$ Tris- $\mathrm{HCl}, \mathrm{pH} 7.5,100 \mathrm{mM}$ $\mathrm{KCl}, 5 \mathrm{mM} \mathrm{MgCl} 2$ ), then heated at $95^{\circ} \mathrm{C}$ for 30 seconds and slow cooled to room temperature (1-2 hours). Cas $9(10 \mathrm{mg} / \mathrm{mL}$, generously gifted by the Seydoux lab) was diluted to $8 \mu \mathrm{M}$ in storage buffer $(20 \mathrm{mM}$ HEPES pH 7.5, $500 \mathrm{mM} \mathrm{KCl)}$. Cas9:tr LNPs were pre-formed by mixing $10 \mu \mathrm{L} 8 \mathrm{uM}$ Cas9 with $10 \mu \mathrm{L} 8 \mu \mathrm{M}$ $\operatorname{tr}_{L}$ and incubating at RT for 10 minutes. RNPs were diluted to $500 \mathrm{pM}-1 \mu \mathrm{M}$ in a 1:1 mix of RNA hybridization buffer and Cas9 storage buffer, then brought to volume with binding buffer and $50 \mathrm{pM}$ substrate in a $20 \mu \mathrm{L}$ reaction. The binding reaction was incubated at $37^{\circ} \mathrm{C}$ for 1 hour, then quenched with $2 \mu \mathrm{L} 6 \mathrm{X}$ purple loading dye (NEB) at RT. $15 \mu \mathrm{L}$ of each reaction was immediately loaded onto an $8 \% \mathrm{TBE}$ gel (Acrylamide/Bis solution 37.5:1, Bio-rad) supplemented with $2 \mathrm{mM} \mathrm{MgCl}_{2}$, and run at $4^{\circ} \mathrm{C}$ at $15 \mathrm{~W}$ in $1 \mathrm{X}$ TBE buffer + $5 \mathrm{mM} \mathrm{MgCl} 2$ for 75-90 minutes. The gel was then removed from its casing, wrapped in plastic wrap and exposed to a phosphor screen overnight. The phosphor screen was scanned and imaged using the Typhoon FLA9500 (GE Healthcare).

\section{In vitro cleavage assay}

$20 \mu \mathrm{L}$ nuclease-free water, $3 \mu \mathrm{L}$ NEBuffer 3.1, $3 \mu \mathrm{L}$ of in vitro transcribed $300 \mathrm{nM}$ sgRNA or tr (produced $^{2}$ as described in in vitro transcription protocol above) and $1 \mu \mathrm{L}$ of $1 \mu \mathrm{M}$ Cas9 nuclease (NEB, M0386S) were mixed and incubated for 10 minutes at $25^{\circ} \mathrm{C}$ to allow for RNP formation. Next, $3 \mu \mathrm{L}$ of a $30 \mathrm{nM}$ dsDNA substrate (a 1kb pCN57 amplicon, amplified from pCN57 with oJW134 and oJW1869) was added, mixed thoroughly, pulsed in a microcentrifuge, and incubated at $37^{\circ} \mathrm{C}$ for 15 minutes. $1 \mu \mathrm{L}$ of proteinase $\mathrm{K}$ (20 $\mathrm{mg} / \mathrm{mL}$, Qiagen) was added and samples were incubated at RT for 10 minutes. 6X loading dye was added, and the reactions were run on a $1.5 \%$ agarose gel.

\section{Competition assay}

To investigate the fitness costs of $\Delta \operatorname{tr}_{L}$, cells harboring a naïve wild-type or $\Delta \operatorname{tr}_{L}$ CRISPR system were cocultured in a long-term competition assay. Wild-type and $\Delta \operatorname{tr}_{L}$ cells were inoculated in $\mathrm{BHI}$ in triplicate and grown overnight at $37^{\circ} \mathrm{C}$, diluted to log phase $\mathrm{OD}=0.1$, and replicate pairs were mixed $1: 1.50 \mu \mathrm{L}$ of a 1:100 dilution of this mixture was immediately plated, and $900 \mu \mathrm{L}$ was frozen at $-80^{\circ} \mathrm{C}$ in $10 \% \mathrm{DMSO}$. The remainder of the mixture was grown to stationary phase in the evening, then diluted back 1:1000 and grown overnight. This continued for a total of 5 days, with frozen stocks taken of the cultures each morning before dilution. To determine the ratio of wild-type: $\Delta \operatorname{tr}_{L}$ cells over time, $5 \mu \mathrm{L}$ of frozen cultures were diluted 1:5000 and plated, and colonies were picked for colony lysis and PCR of the tracrRNA locus (oJW2012/oJW1447). Amplicon length (314 bp for wild-type and $262 \mathrm{bp}$ for $\Delta \operatorname{tr}_{\mathrm{L}}$ ) allowed us to estimate the relative abundance of each strain in the mixed culture over time.

\section{Evolutionary analysis}

Bacterial strains harboring type II-A CRISPR-Cas systems with previously annotated short-form tracrRNAs ${ }^{8}$ were investigated for evidence of $\operatorname{rr}_{L}$ repression. Accession numbers for species investigated are listed in Table S2 along with all metadata compiled in our analysis. The genomic locus between the annotated 3' end of tracrRNA and the translational start site of Cas9 was interrogated using the DSK k-mer counting software ${ }^{9}$ with $\mathrm{k}$-mer length set to $11 \mathrm{bp}$ and a minimal match frequency of 2 . We classified strains as 
" $\mathrm{tr}_{\mathrm{L}}{ }^{+\prime}$ if one $\mathrm{k}$-mer instance was on the same strand as $\operatorname{tr}_{\mathrm{S}}$ followed immediately by a 5'-GTTTTA-3' sequence allowing for 1 mismatch (representing the targeting site within $\operatorname{tr}_{\mathrm{L}}$ ) and the other instance was upstream of site 1, immediately followed by a 5'-NGG-3' sequence allowing for 1 mismatch (representing the targeted site within $\left.\mathrm{P}_{\text {cas }}\right)$. We manually inspected the $16 \operatorname{tr}_{\mathrm{L}}{ }^{-}$loci with intergenic lengths above $167 \mathrm{bp}$ for candidate $\operatorname{tr}_{\mathrm{L}}$ targeting sites with a single seed mismatch but perfect lower stems and PAMs, and found one additional trL sequence with a $10 \mathrm{bp}$ match.

We aligned tracrRNA sequences using $\mathrm{MAFFT}^{10}$ (default parameters). Cas 9 amino acid sequences for these strains were aligned using MUSCLE ${ }^{11}$, and a phylogenetic tree was constructed using FastTree, with the WAG evolutionary model ${ }^{12}$. All multiple sequence alignments and tree generation were performed in Geneious Prime 2020.1.1.

\section{References for Supplementary Methods}

1 Gibson, D. G. et al. Enzymatic assembly of DNA molecules up to several hundred kilobases. Nature Methods 6, 343-U341, doi:10.1038/Nmeth.1318 (2009).

2 Youngman, P. J., Perkins, J. B. \& Losick, R. Genetic transposition and insertional mutagenesis in Bacillus subtilis with Streptococcus faecalis transposon Tn917. Proc Natl Acad Sci U S A 80, 2305-2309, doi:10.1073/pnas.80.8.2305 (1983).

3 Robinson, J. T. et al. Integrative genomics viewer. Nat Biotechnol 29, 24-26, doi:10.1038/nbt.1754 (2011).

4 Heler, R. et al. Cas9 specifies functional viral targets during CRISPR-Cas adaptation. Nature 519, 199-202, doi:10.1038/nature14245 (2015).

5 Modell, J. W., Jiang, W. \& Marraffini, L. A. CRISPR-Cas systems exploit viral DNA injection to establish and maintain adaptive immunity. Nature 544, 101-104, doi:10.1038/nature21719 (2017).

6 Fields, C., Sheng, P., Miller, B., Wei, T. \& Xie, M. Northern Blot with IR Fluorescent Probes: Strategies for Probe Preparation. Bio Protoc 9, doi:10.21769/BioProtoc.3219 (2019).

7 Culviner, P. H., Guegler, C. K. \& Laub, M. T. A Simple, Cost-Effective, and Robust Method for rRNA Depletion in RNA-Sequencing Studies. mBio 11, doi:10.1128/mBio.00010-20 (2020).

8 Faure, G. et al. Comparative genomics and evolution of trans-activating RNAs in Class 2 CRISPR-Cas systems. RNA Biol 16, 435-448, doi:10.1080/15476286.2018.1493331 (2019).

9 Rizk, G., Lavenier, D. \& Chikhi, R. DSK: k-mer counting with very low memory usage. Bioinformatics 29, 652-653, doi:10.1093/bioinformatics/btt020 (2013).

10 Katoh, K., Misawa, K., Kuma, K. \& Miyata, T. MAFFT: a novel method for rapid multiple sequence alignment based on fast Fourier transform. Nucleic Acids Res 30, 3059-3066, doi:10.1093/nar/gkf436 (2002).

11 Edgar, R. C. MUSCLE: a multiple sequence alignment method with reduced time and space complexity. BMC Bioinformatics 5, 113, doi:10.1186/1471-2105-5-113 (2004).

12 Price, M. N., Dehal, P. S. \& Arkin, A. P. FastTree 2--approximately maximum-likelihood trees for large alignments. PLoS One 5, e9490, doi:10.1371/journal.pone.0009490 (2010). 


\section{References for Supplementary Materials}

$1 \quad$ Kreiswirth, B. N. et al. The toxic shock syndrome exotoxin structural gene is not detectably transmitted by a prophage. Nature 305, 709-712, doi:10.1038/305709a0 (1983).

2 Horinouchi, S. \& Weisblum, B. Nucleotide sequence and functional map of pC194, a plasmid that specifies inducible chloramphenicol resistance. J Bacteriol 150, 815-825 (1982).

3 Horinouchi, S. \& Weisblum, B. Nucleotide sequence and functional map of pE194, a plasmid that specifies inducible resistance to macrolide, lincosamide, and streptogramin type B antibodies. J Bacteriol 150, 804-814 (1982).

4 Charpentier, E. et al. Novel cassette-based shuttle vector system for gram-positive bacteria. Appl Environ Microbiol 70, 6076-6085, doi:10.1128/AEM.70.10.60766085.2004 (2004).

5 Goldberg, G. W., Jiang, W., Bikard, D. \& Marraffini, L. A. Conditional tolerance of temperate phages via transcription-dependent CRISPR-Cas targeting. Nature 514, 633-637, doi:10.1038/nature13637 (2014).

6 Heler, R. et al. Cas9 specifies functional viral targets during CRISPR-Cas adaptation. Nature 519, 199-202, doi:10.1038/nature14245 (2015).

7 Modell, J. W., Jiang, W. \& Marraffini, L. A. CRISPR-Cas systems exploit viral DNA injection to establish and maintain adaptive immunity. Nature 544, 101-104, doi:10.1038/nature21719 (2017).

8 Devos, W. M. Gene Cloning in Lactic Streptococci. Neth Milk Dairy J 40, 141-154 (1986).

9 Khan, S. A. \& Novick, R. P. Complete nucleotide sequence of pT181, a tetracyclineresistance plasmid from Staphylococcus aureus. Plasmid 10, 251-259, doi:10.1016/0147-619x(83)90039-2 (1983).

10 Youngman, P. J., Perkins, J. B. \& Losick, R. Genetic transposition and insertional mutagenesis in Bacillus subtilis with Streptococcus faecalis transposon Tn917. Proc Natl Acad Sci U S A 80, 2305-2309, doi:10.1073/pnas.80.8.2305 (1983). 
bioRxiv preprint doi: https://doi.org/10.1101/2020.05.21.102756; this version posted May 21, 2020. The copyright holder for this preprint (which

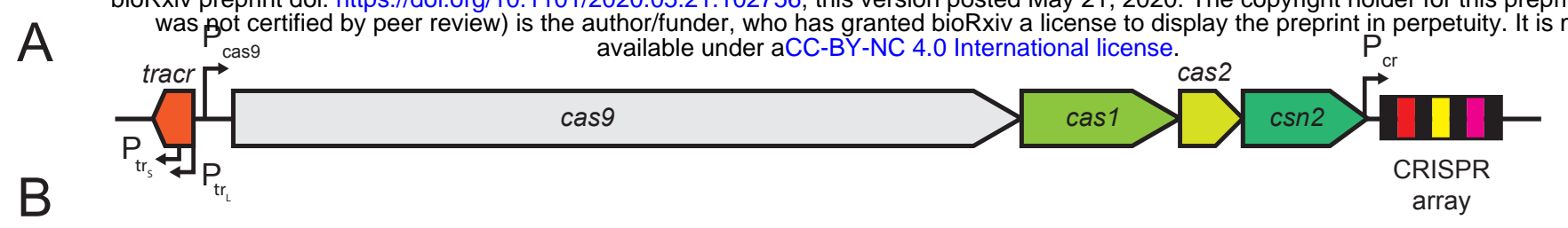

Fig. 1

B

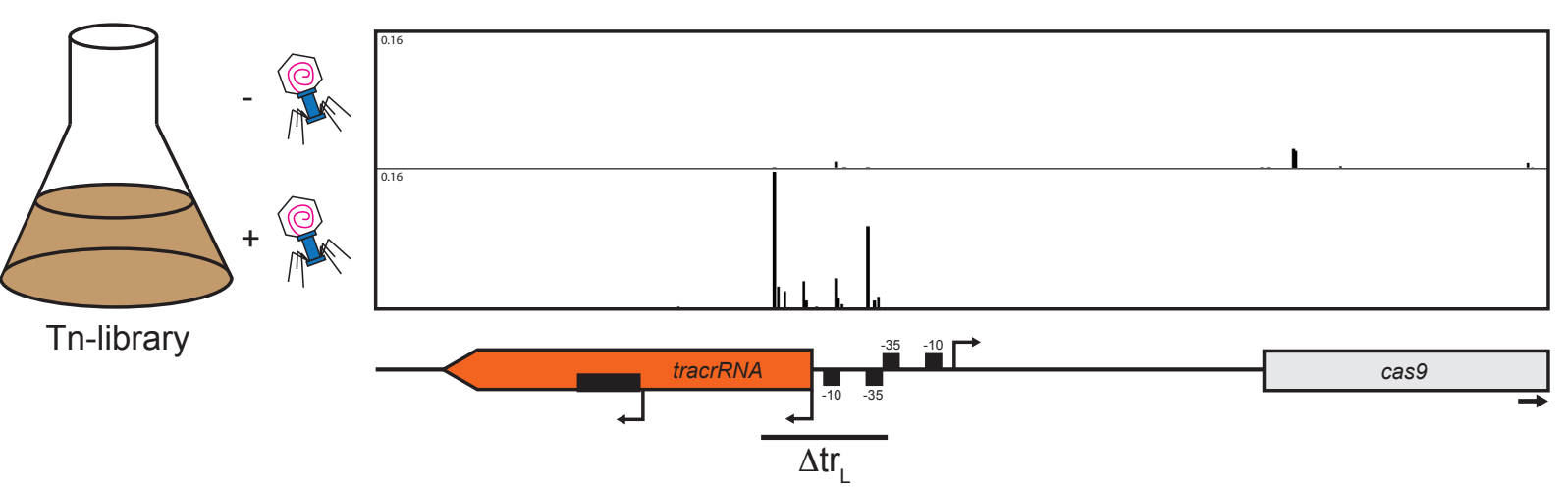

C

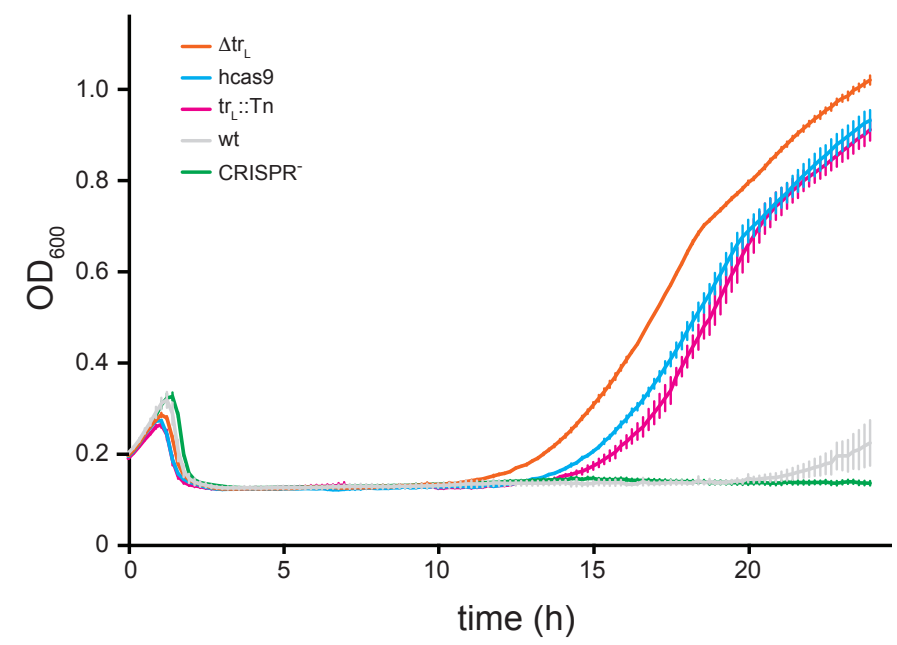

D

E
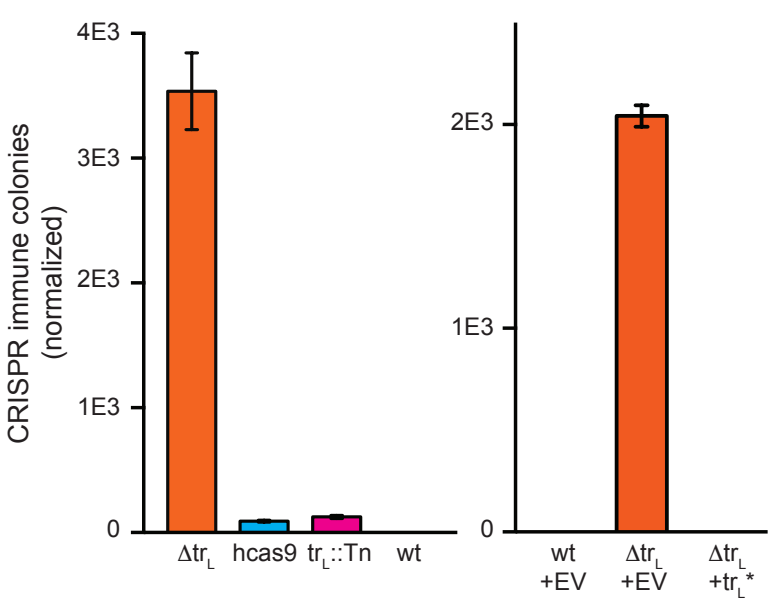
bioRxiv preprint doi: https://doi.org/10.1101/2020.05.21.102756; this version posted May 21, 2020. The copyright holder for this preprint (which was not certified by peer review) is the author/funder, who has granted bioRxiv a license to display the preprint in perpetuity. It is made available under aCC-BY-NC 4.0 International license.

A

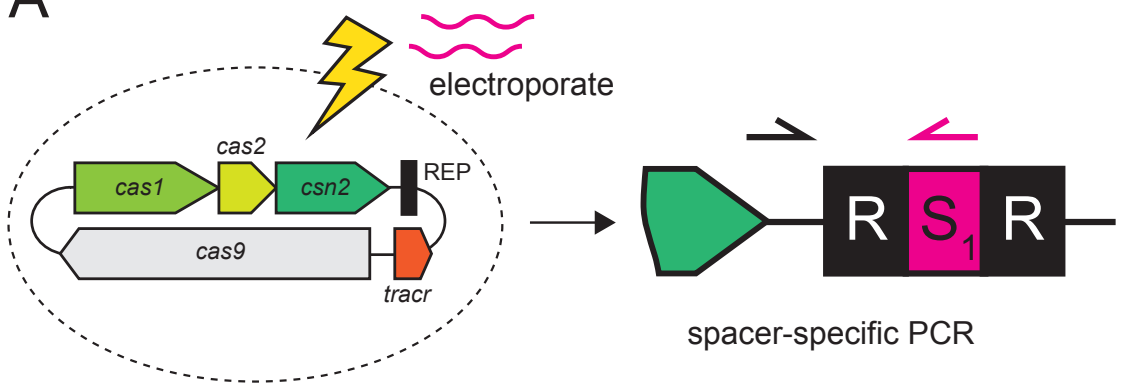

B

C

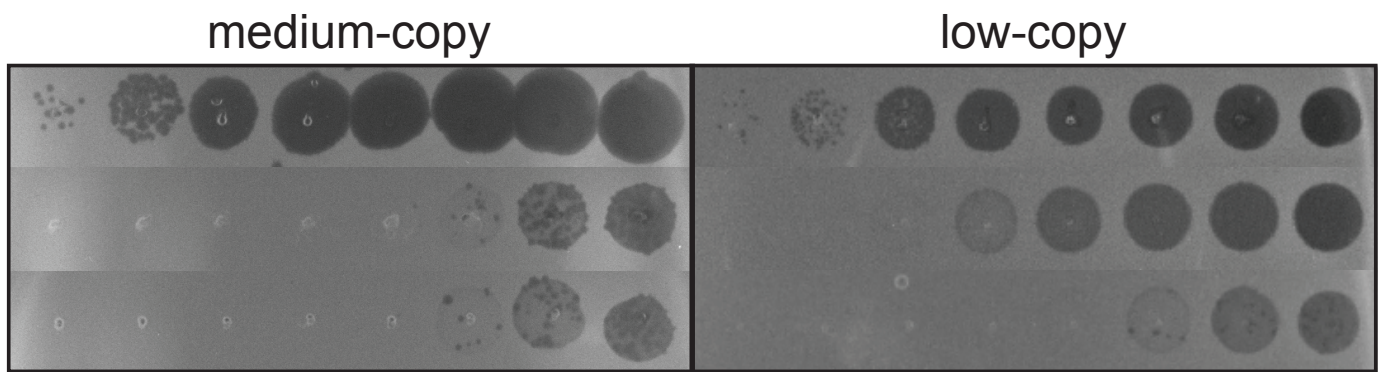

tracr spacer

WT NT

WT $\quad \alpha-\phi N M 4$

$\Delta \operatorname{tr}_{\mathrm{L}} \quad \alpha-\phi N M 4$

D

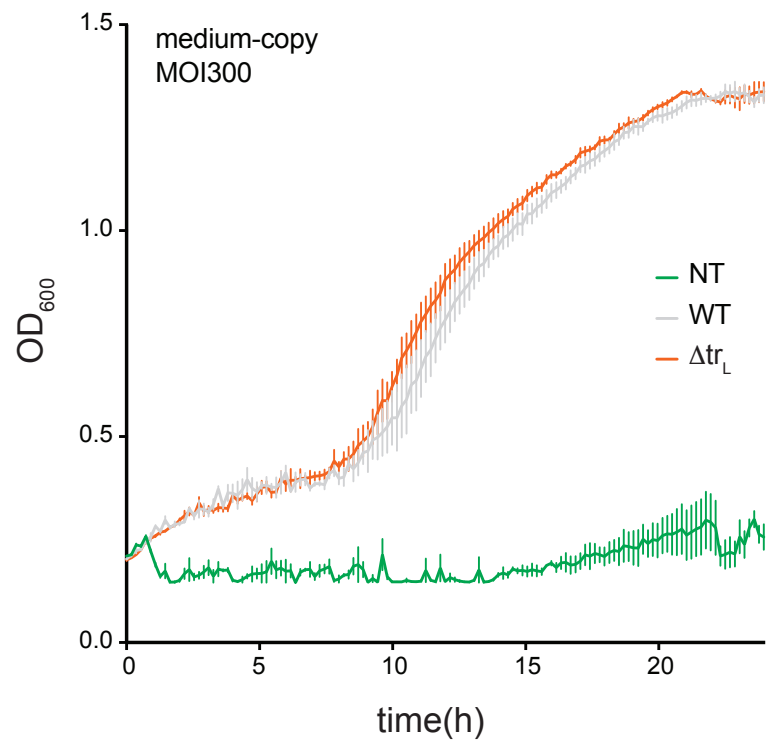

E

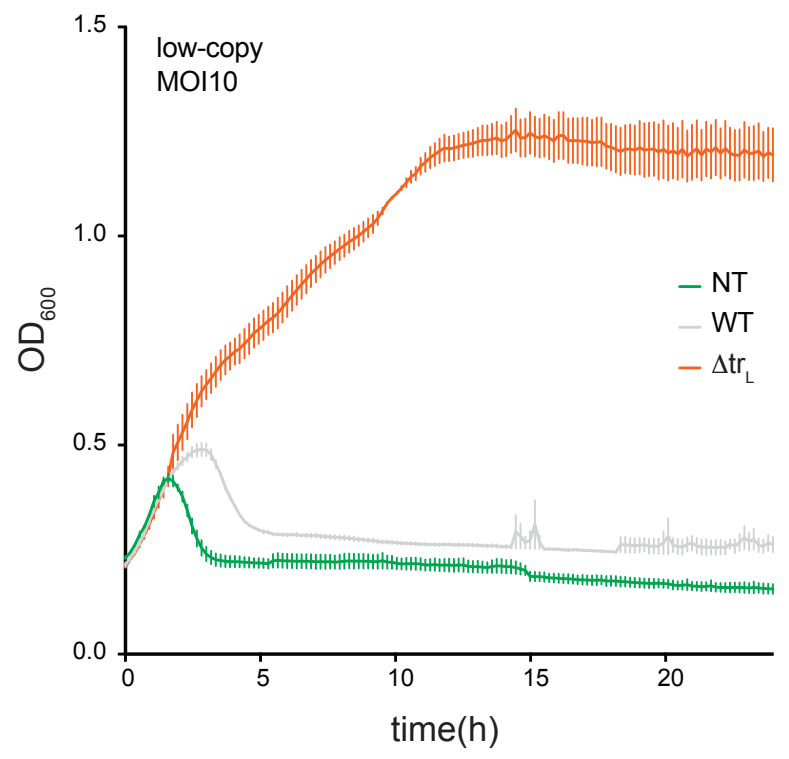


bioRxiv preprint doi: https://doi.org/10.1101/2020.05.21.102756; this version posted May 21,2020 . The copyright holder for this preprint (which
was not certified by peer review) is the author/funder, who has aranted bioRxiv a license to displav the preprint in perpetuity. It is made

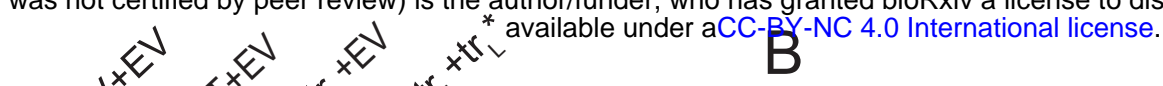

Fig. 3

A
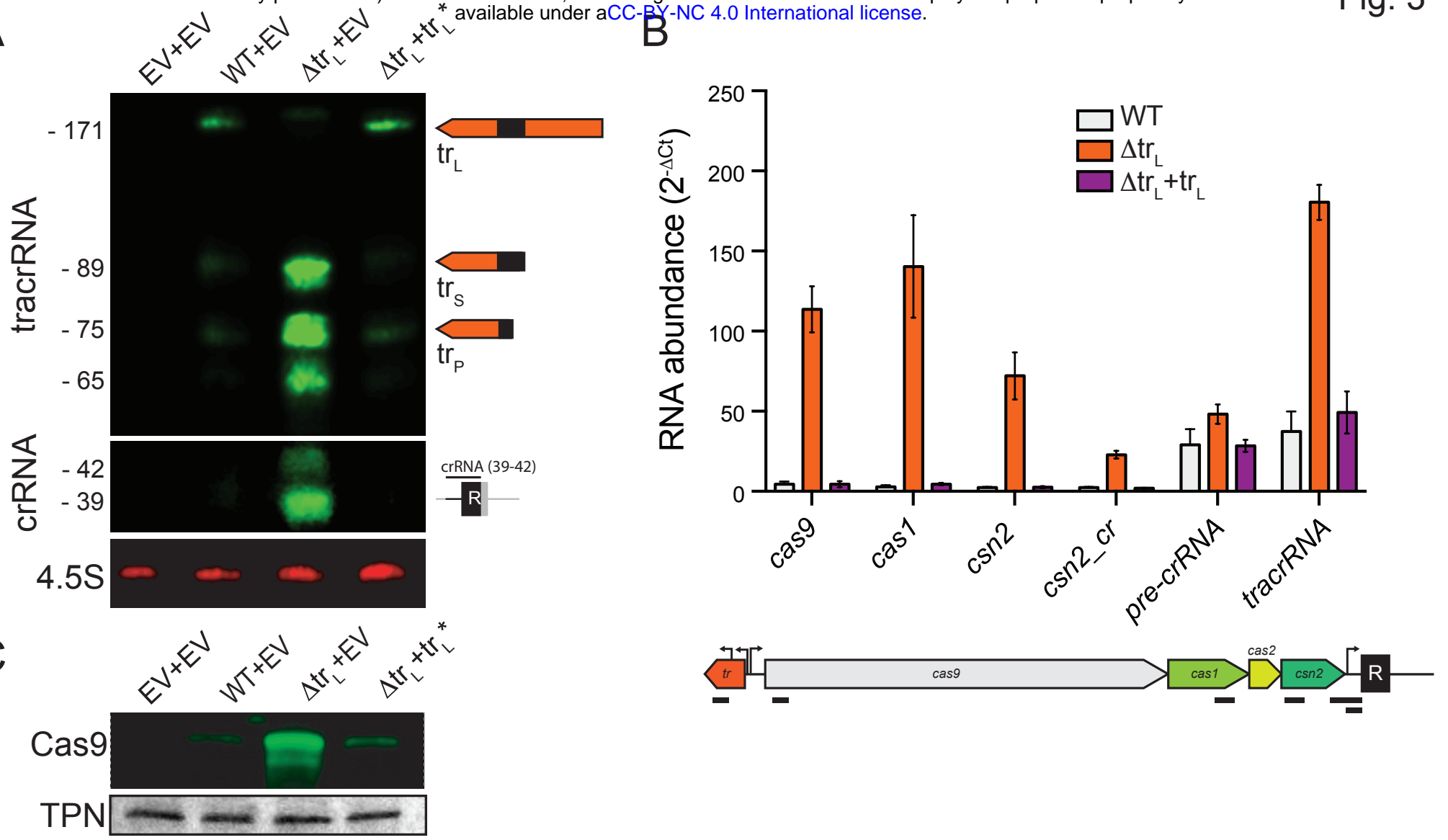
bioRxiv preprint doi: https://doi.org/10.1101/2020.05.21.102756; this version posted May 21, 2020. The copyright holder for this preprint (which

was not certified by peer review) is the author/funder, who has granted bioRxiv a license to display the preprint in perpetuity. It is made available under aCC-BY-NC 4.0 International license.

A

B
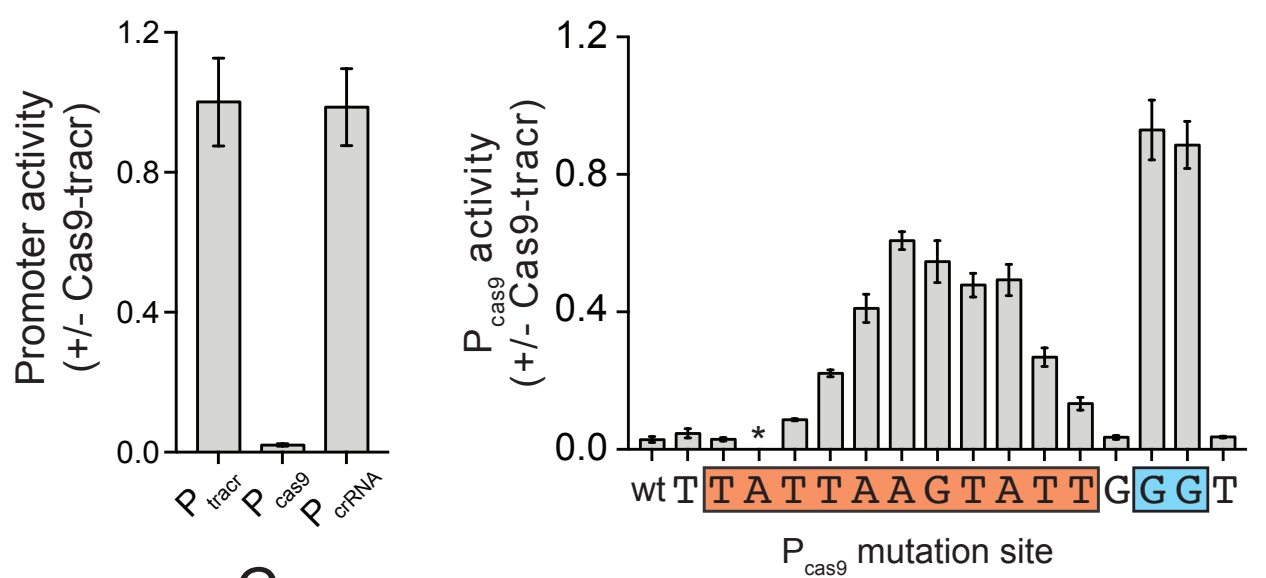

D
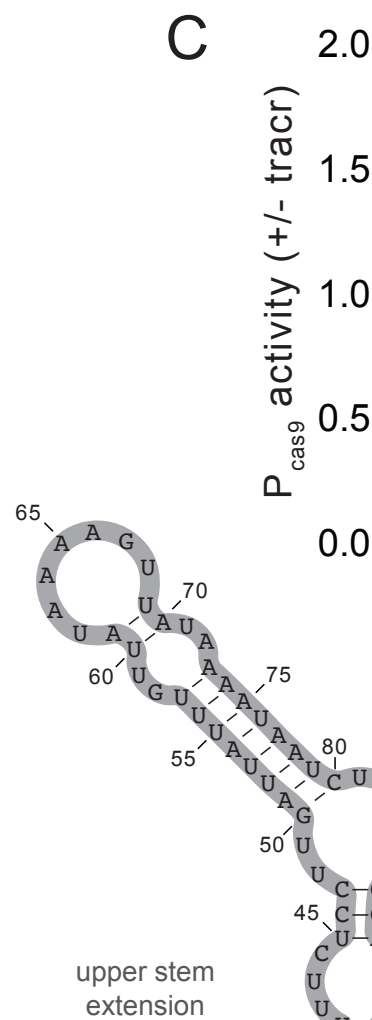

$\mathrm{Q}^{\circ}$

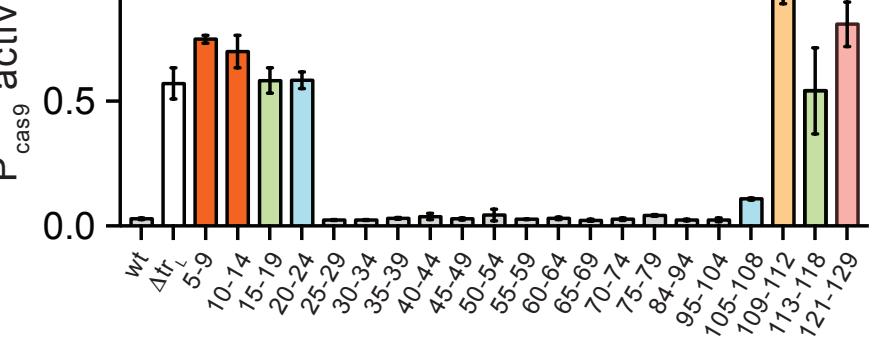

tracrRNA mutation site

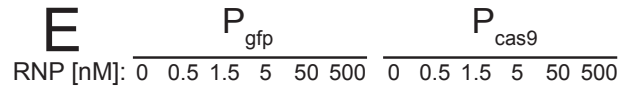

extension
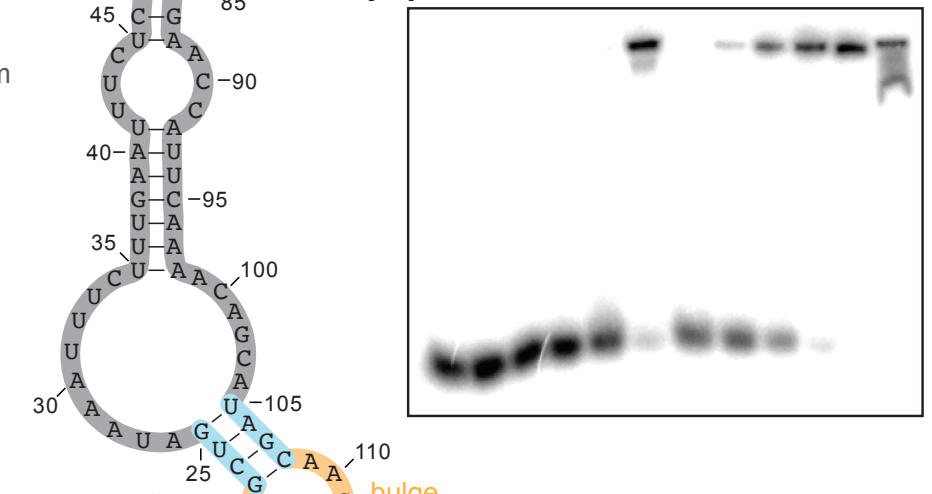

Gulge

$20^{-U}{ }^{A}-U^{U}$

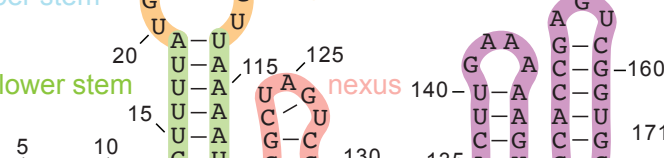

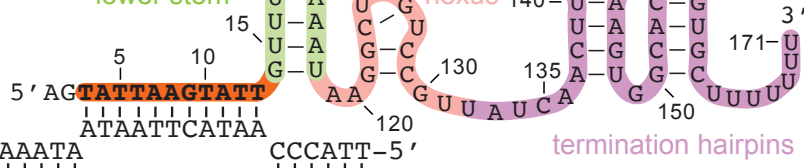

3'-CAATATTAGATAAATA

5 ' -GTIATAATCTATTTATTATTAAGTATTGGGTAA-3'

$P_{\text {cas9 }}$

PAM 
bioRxiv preprint doi: https://doi.org/10.1101/2020.05.21.102756; this version posted May 21, 2020. The copyright holder for this preprint (which was not certified by peer review) is the author/funder, who has granted bioRxiv a license to display the preprint in perpetuity. It is made

A available under aCC-BY-NC 4.0 International license.

$B$
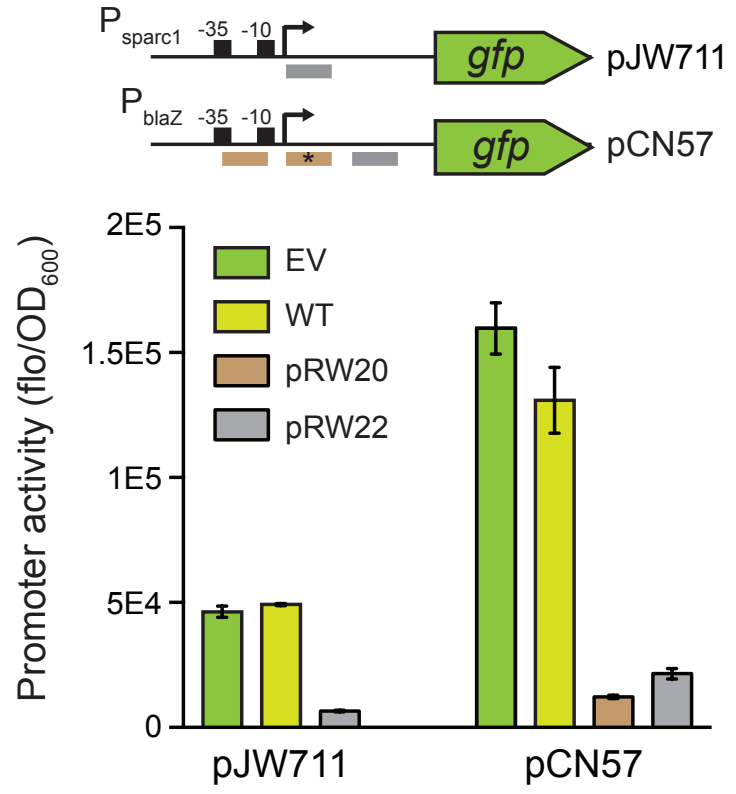

Template +++++++++ Cas9 ++++++++ $\begin{array}{llllllllll}\operatorname{tr}_{\mathrm{L}} & \mathrm{WT} & 11 & 13 & 15 & 17 & 19 & \mathrm{SG} 1 \mathrm{SG} 2 & - & -\end{array}$ 
bioRxiv preprint doi: https://doi.org/10.1101/2020.05.21.102756; this version posted May 21, 2020. The copyright holder for this preprint (which was not certified by peer review) is the author/funder, who has granted bioRxiv a license to display the preprint in perpetuity. It is made A available under aCC-BY-NC 4.0 International license.

Fig. 6
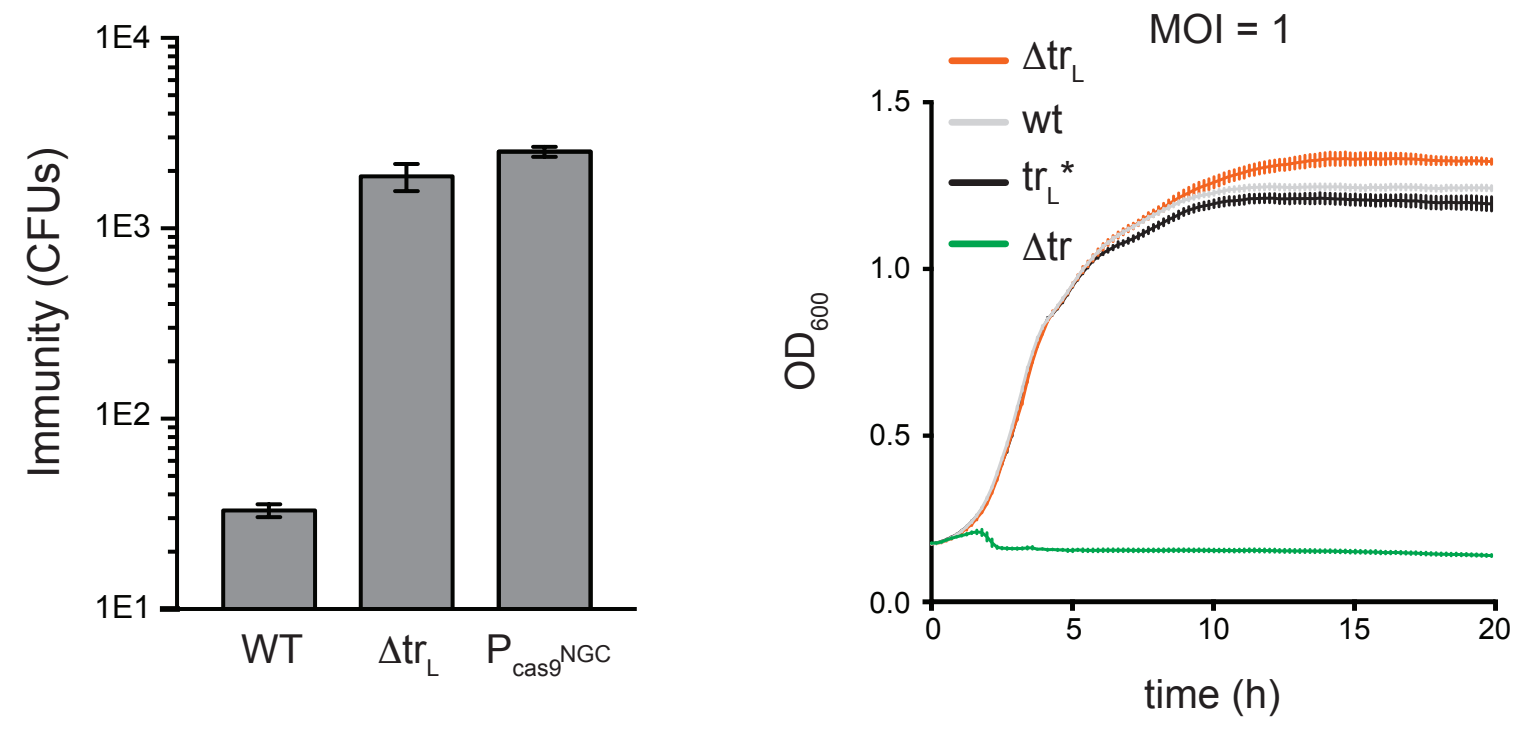

C

$\begin{array}{llllllll}\text { EV } & \text { SR } & \text { Sp1 } & \text { Sp2 } & \text { Sp3 } & \text { Sp4 } & \text { Sp5 } & \text { Sp6 }\end{array}$
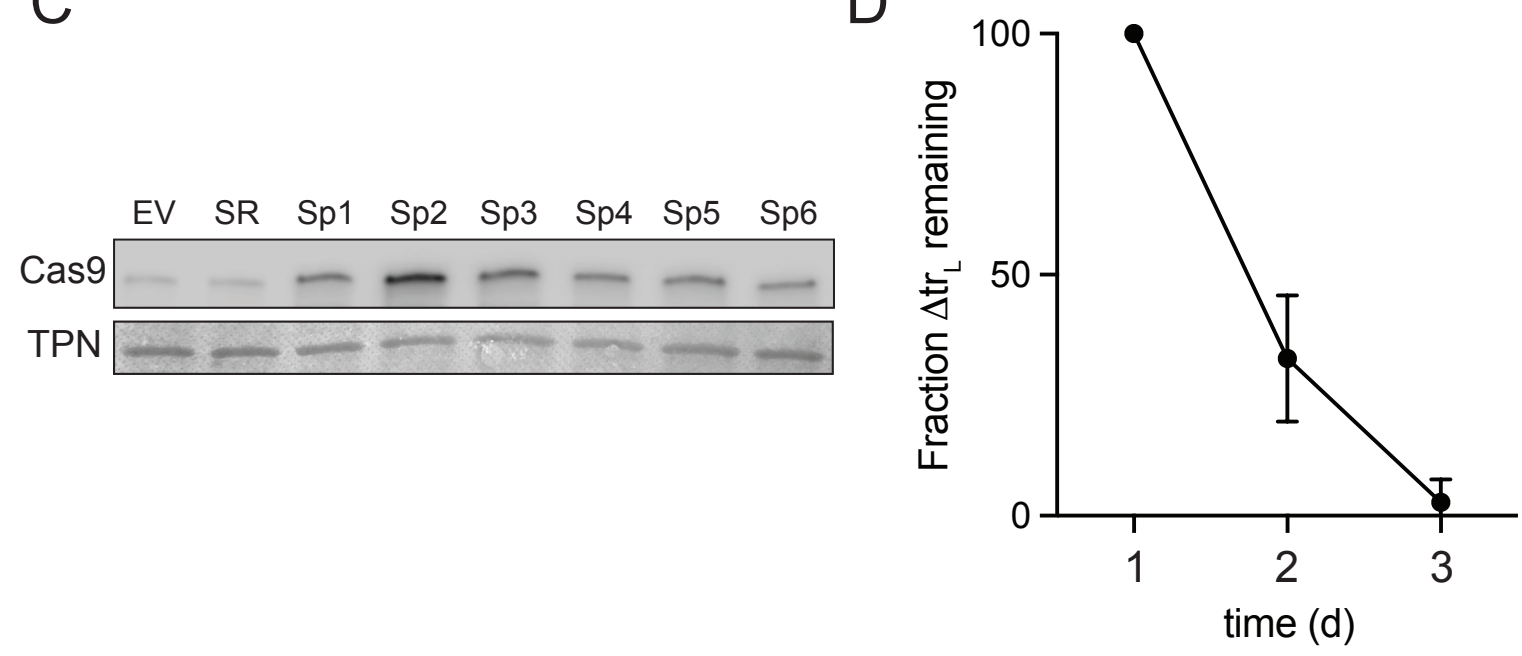

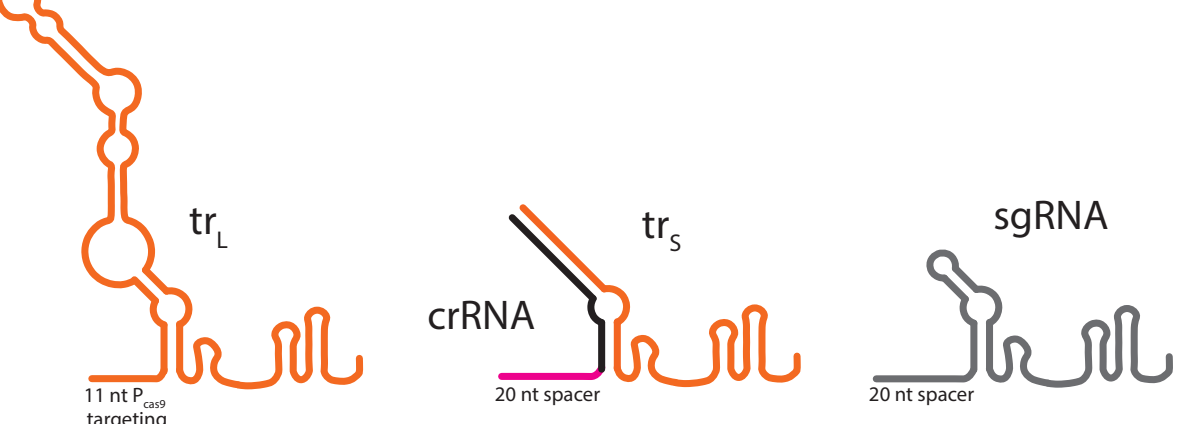

B
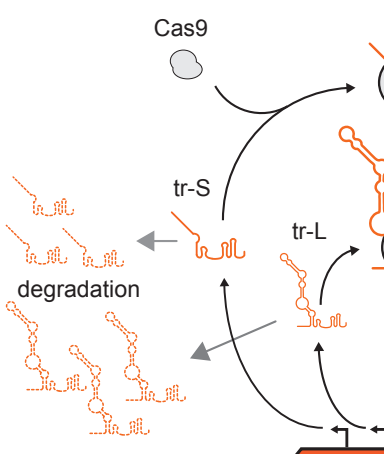

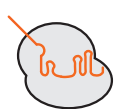

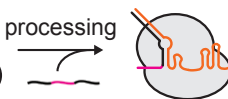
r-S

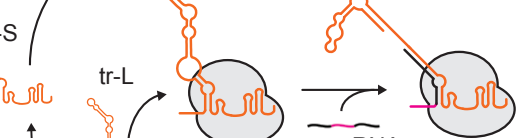
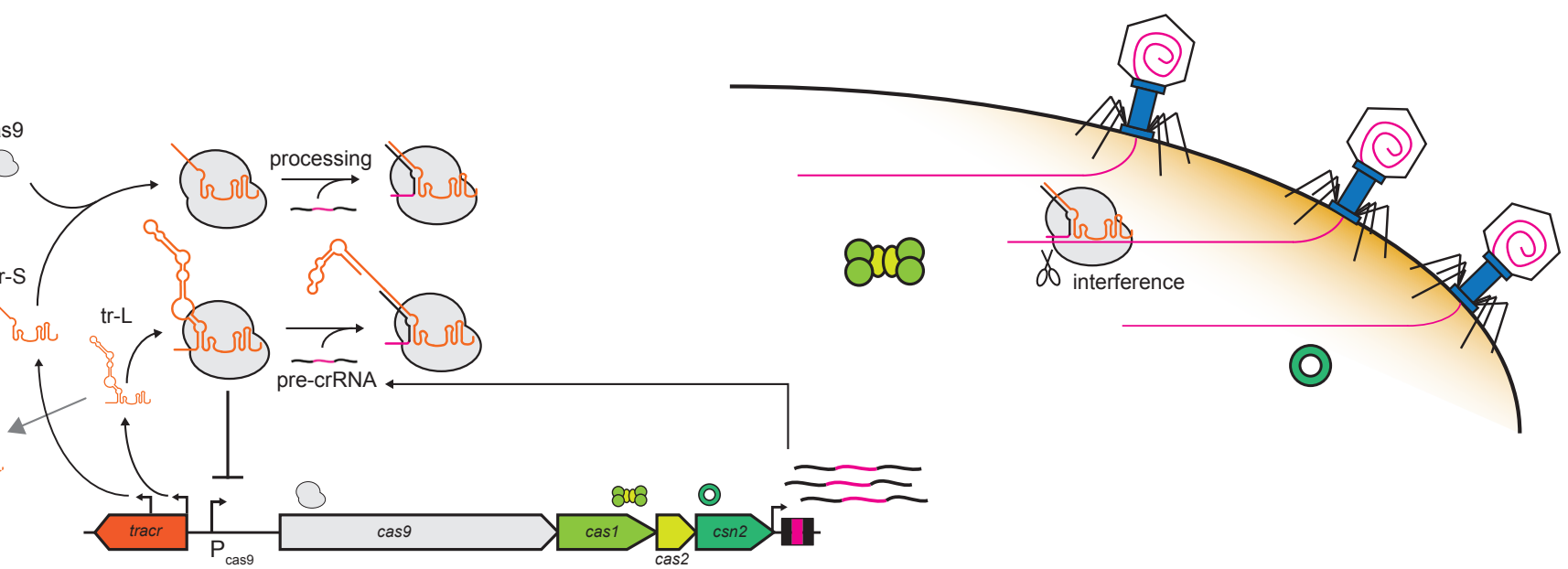

C
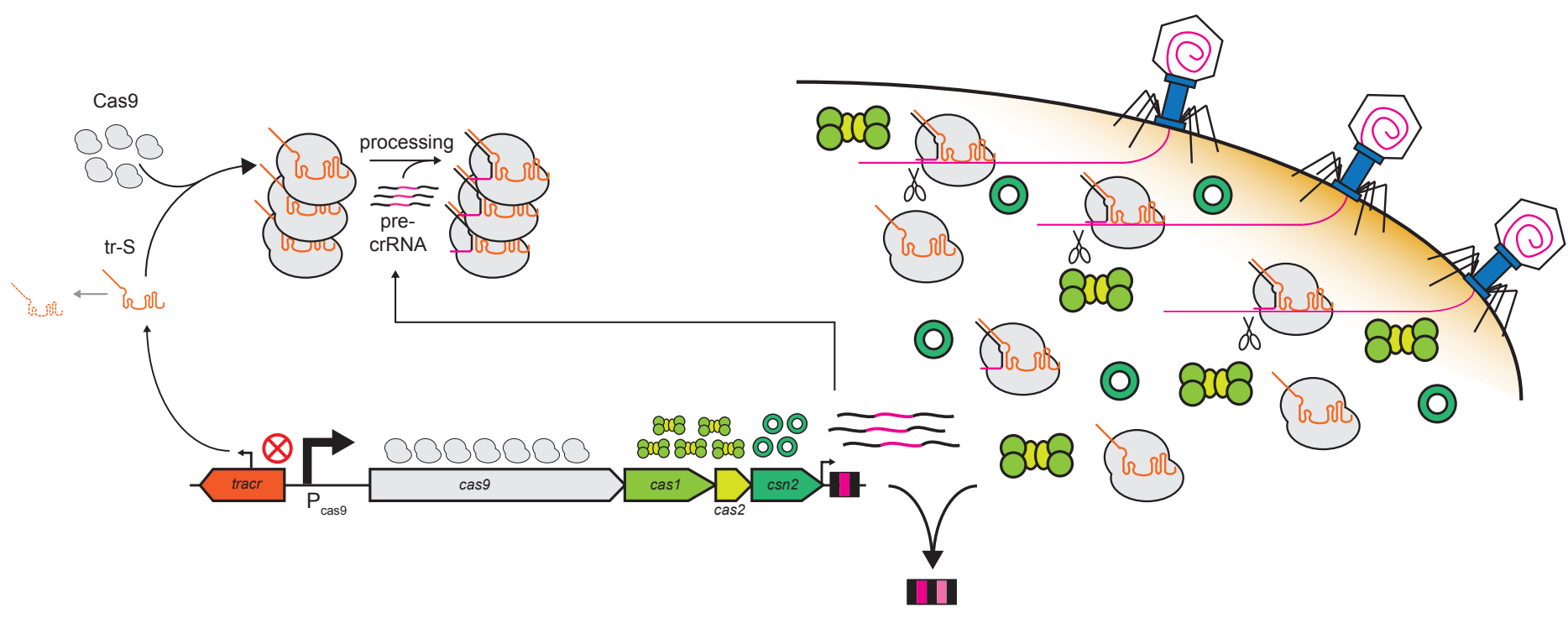

adaptation 
$\mathrm{R}$ repeat

spacer

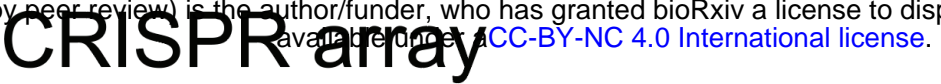

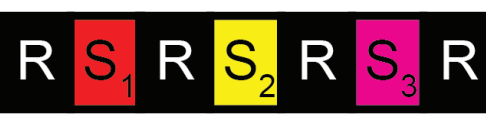

transcription $\downarrow$

pre-crRNA

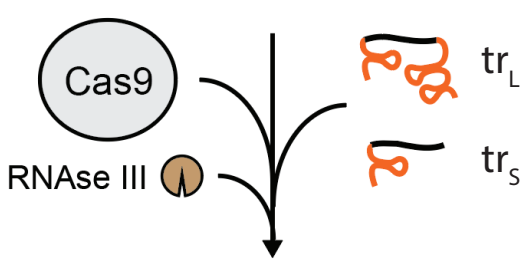

complementary to

crRNA repeat

$1^{\circ}$ processing

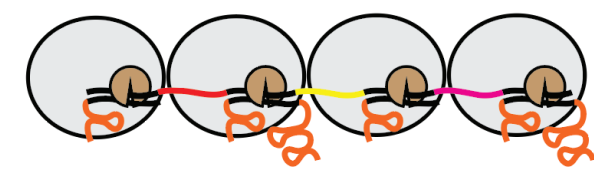

$\rightarrow \sim 2$

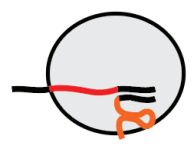

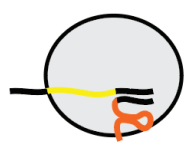

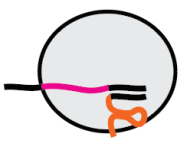

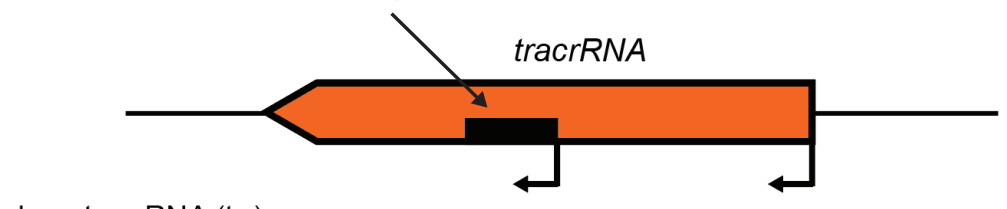

long tracrRNA $\left(\operatorname{tr}_{L}\right)$

short tracrRNA (trs $)$

\section{mature targeting complex}
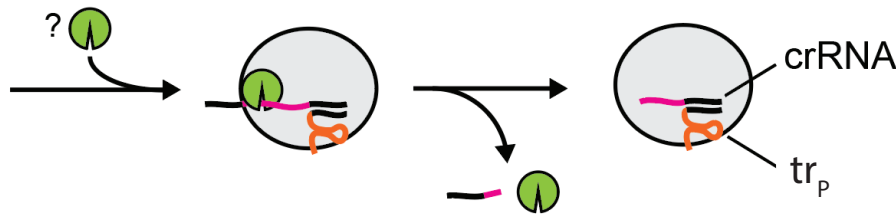

$2^{\circ}$ processing

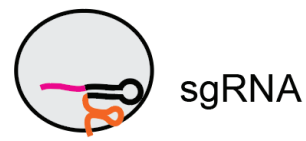




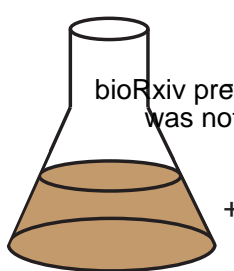

Tn-library

(2)

prialdoi: https://doi.org/10.1101/2020.05.21.102756; this version posted May 21, 2020. The copyright holder for this preprint (which $+$

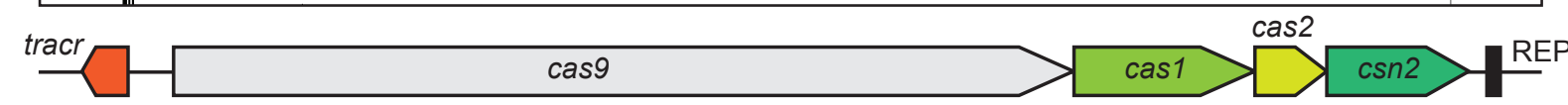

B

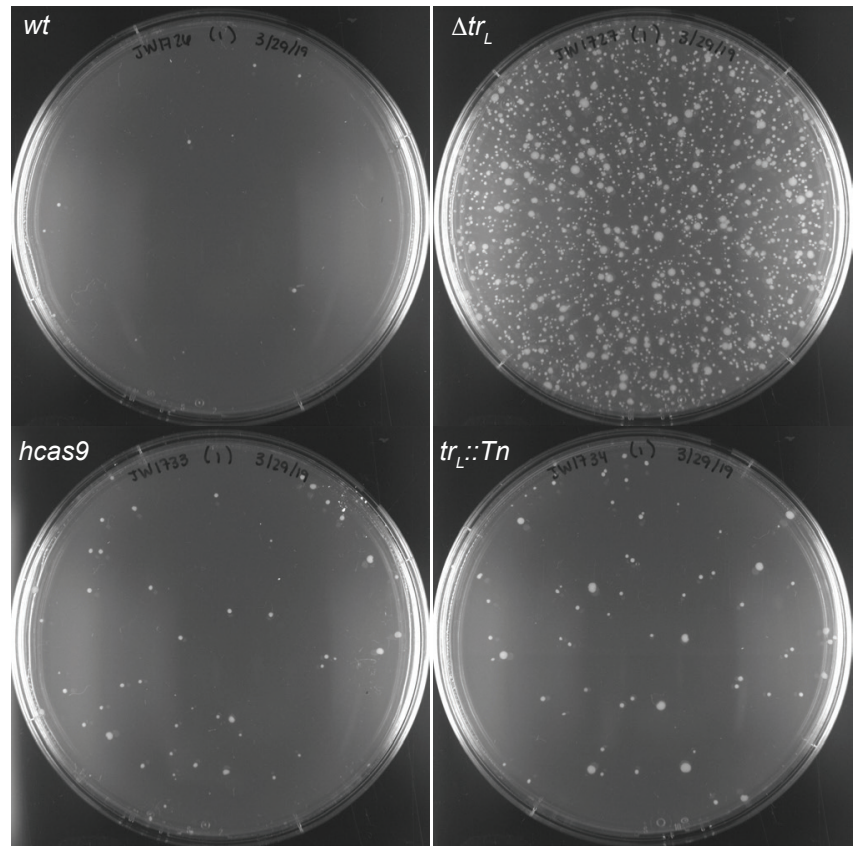

C

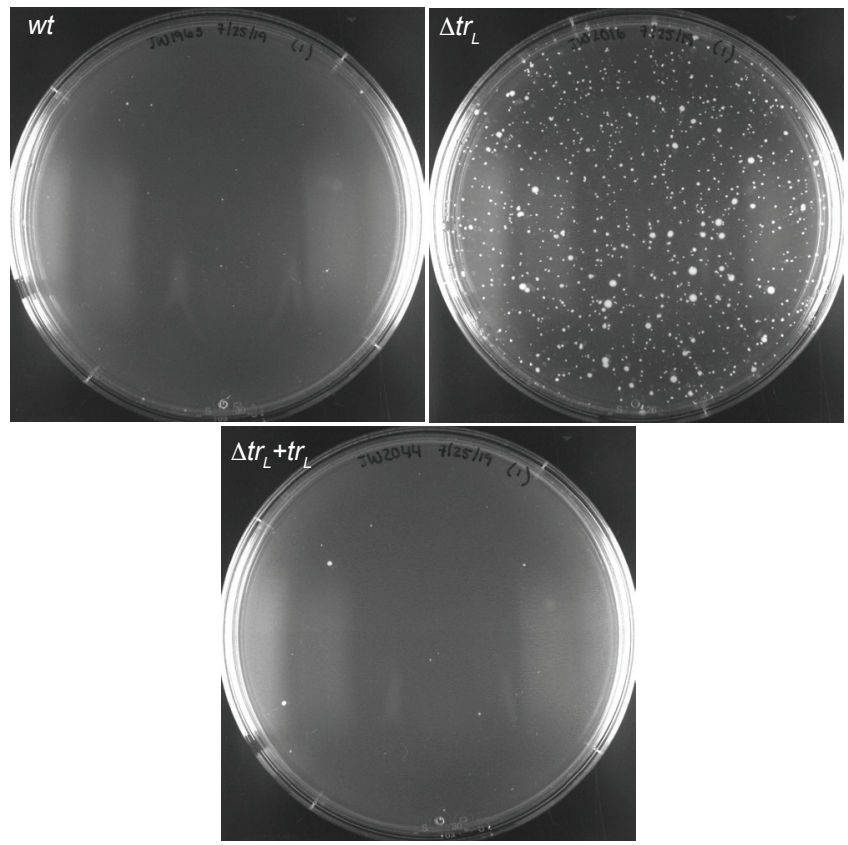

$\mathrm{D}$

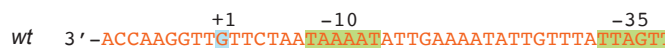

$+1 \quad-10$

$-35$

tr. ${ }^{*}$ '

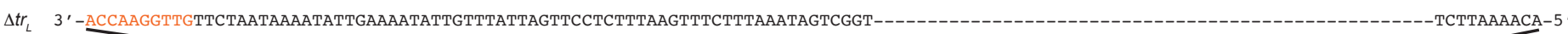

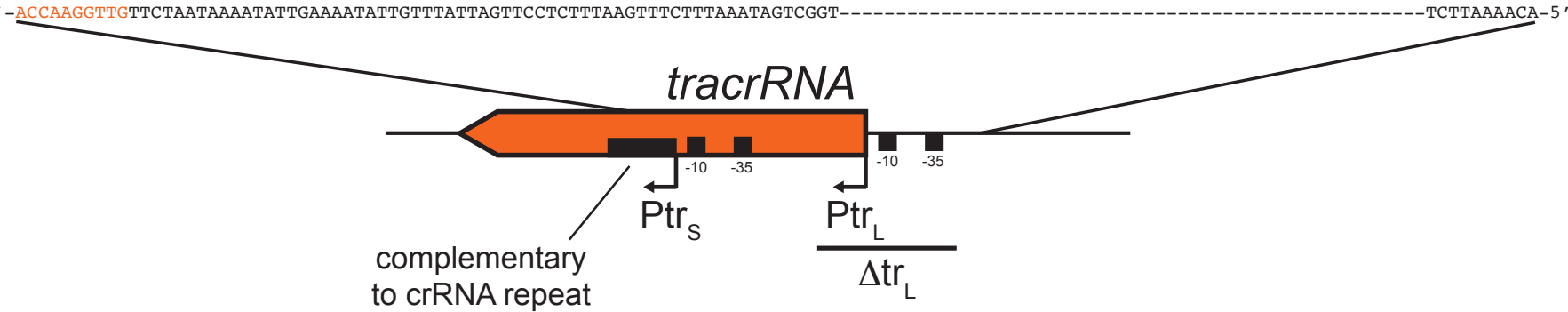

$\mathrm{E}$

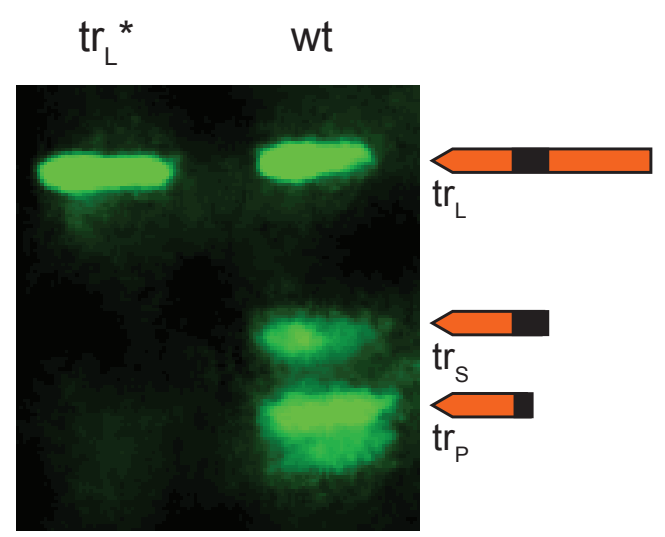


bioRxiv preprint doi: https://doi.org/10.1101/2020.05.21.102756; this version posted May 21, 2020. The copyright holder for this preprint (which

was not certified by peer review) is the author/funder, who has granted bioRxiv a license to display the preprint in perpetuity. It is made available under aCC-BY-NC 4.0 International license.

Fig. S3

A

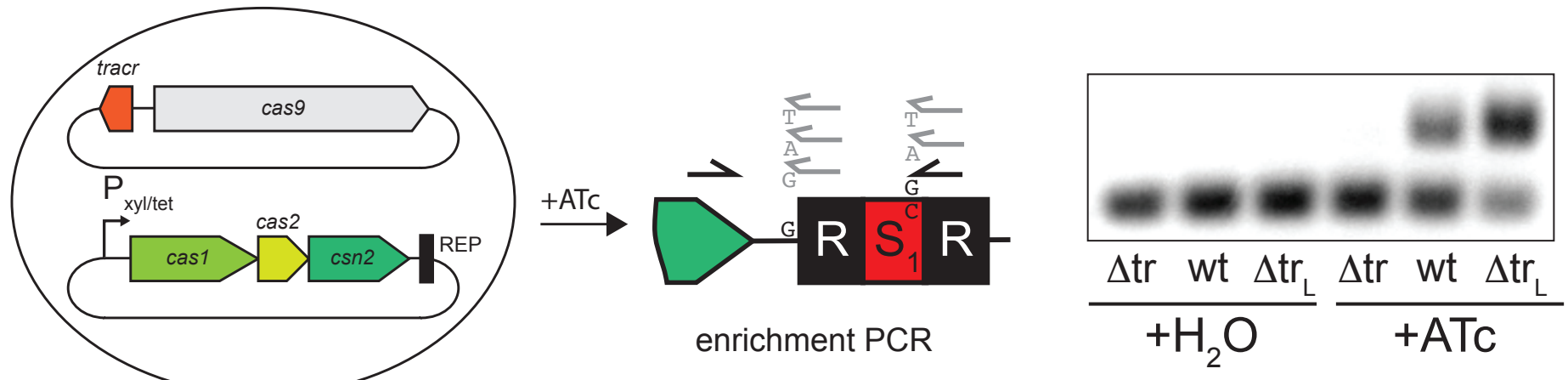

B
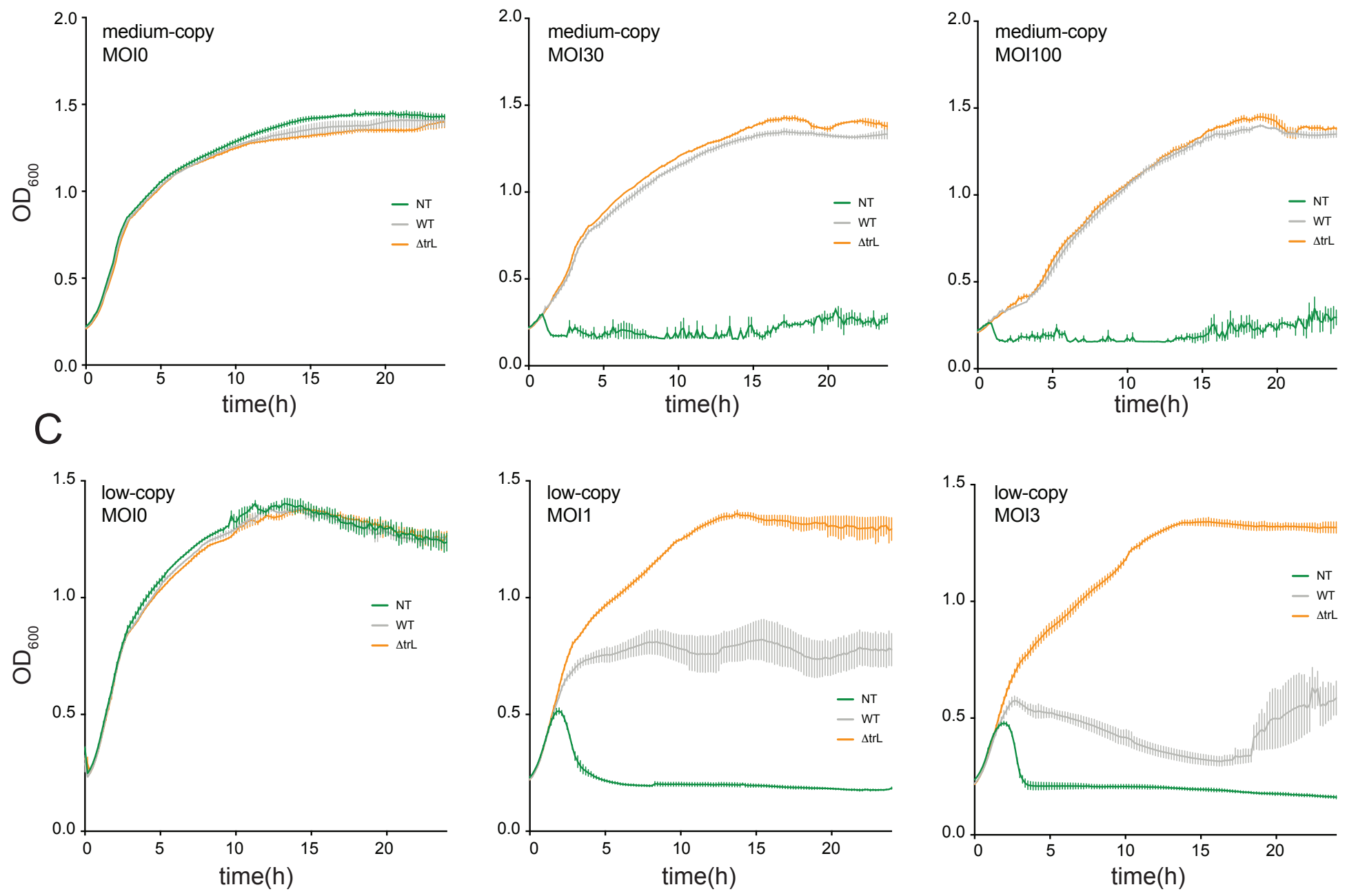
bioRxiv preprint doi: https://doi.org/10.1101/2020.05.21.102756; this version posted May 21, 2020. The copyright holder for this preprint (which

A was not certified by peer review) is the author/funder, who has granted bioRxiv a license to display the preprint in perpetuity. It is made available under aCC-BY-NC 4.0 International license.

Fig. S4

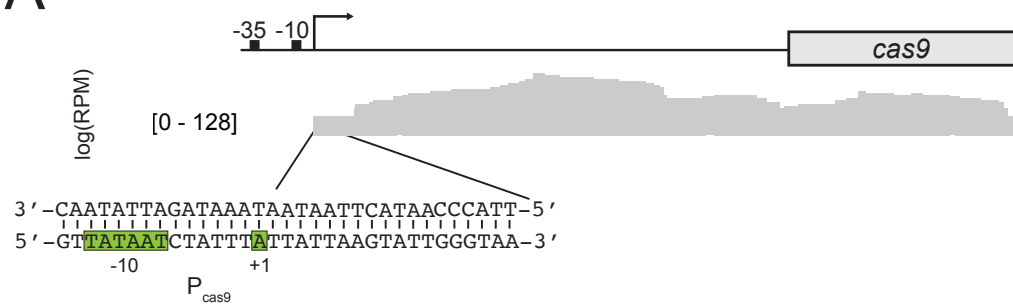

B

wt

$[0-7091]$

[0 - 66143]

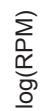

$\Delta \operatorname{tr}_{\mathrm{L}}$

[0 - 152290]

[0 - 114145]

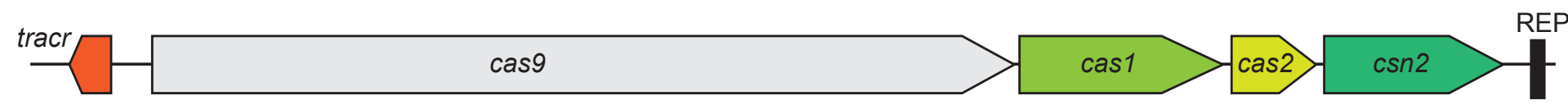

C

$\frac{\log }{W^{T} \Delta^{T_{2}}} \frac{\text { Stat }}{W^{T} \quad \Delta^{T_{2}}}$

$\frac{}{\frac{1}{2}}$

$-171$

$-89$

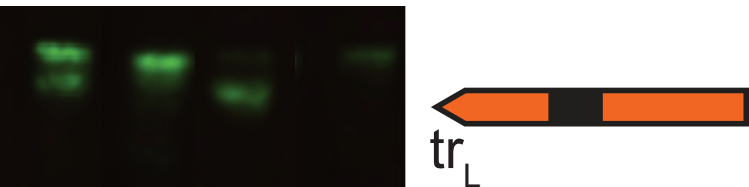

$-75$

$-65$

$$
-75
$$

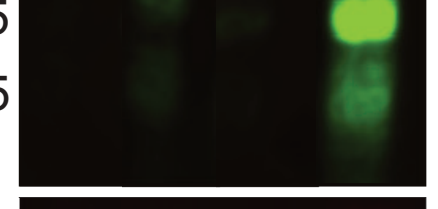

$4.5 \mathrm{~S}$
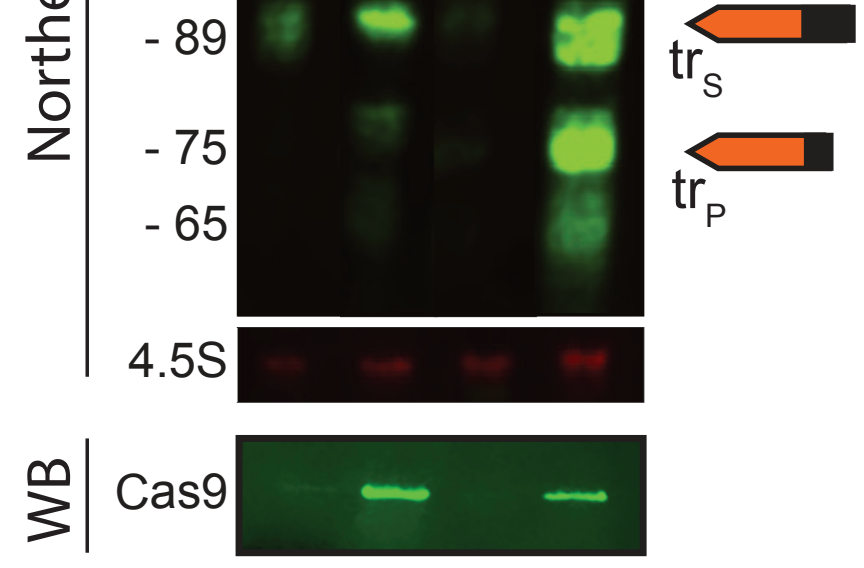
bioRxiv preprint doi: https://doi.org/10.1101/2020.05.21.102756; this version posted May 21, 2020. The copyright holder for this preprint (which

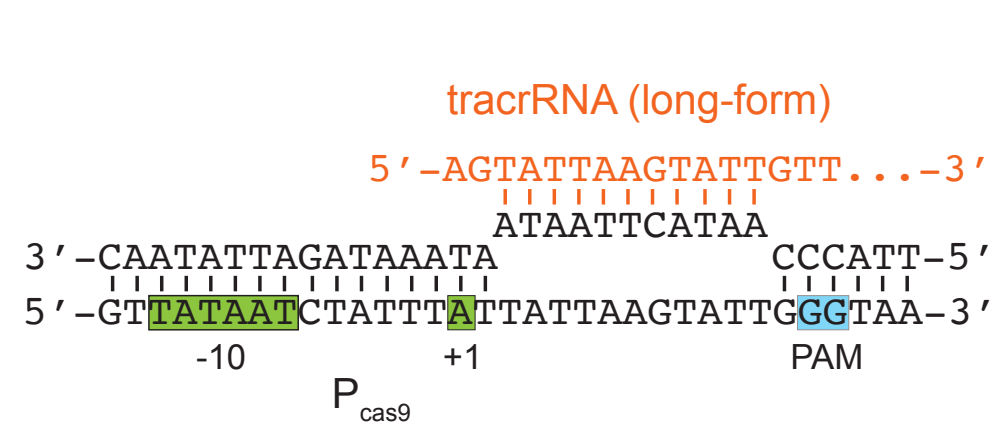

C

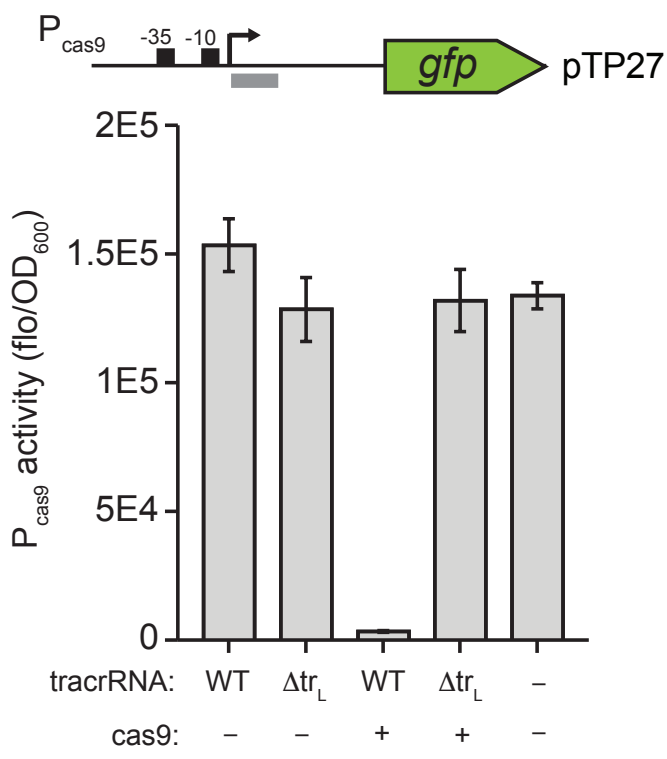

$E$
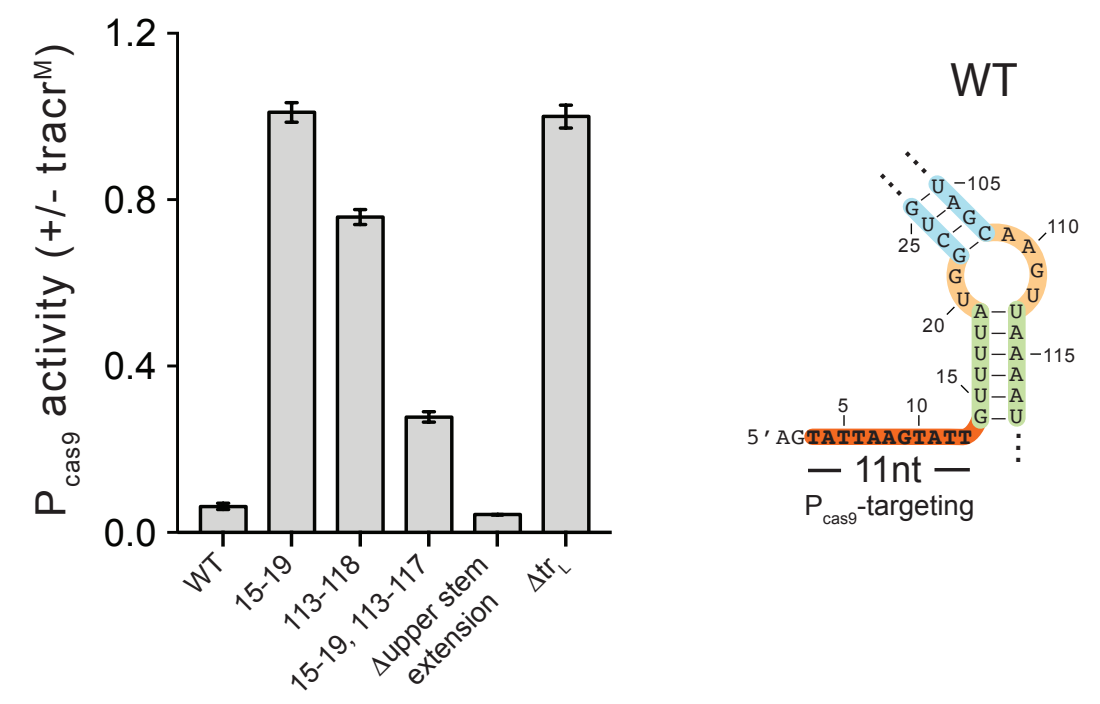

$\mathrm{F}$

WT

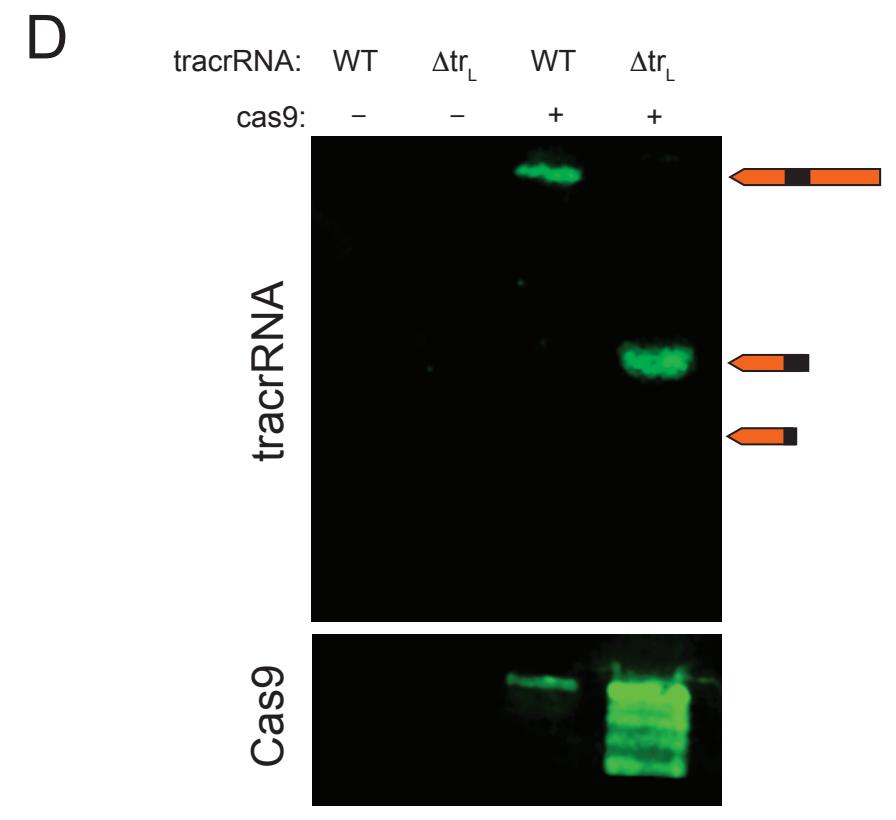

$P_{\text {cas } 9}$ mutation site

15-19,

15-19 113-118 113-117

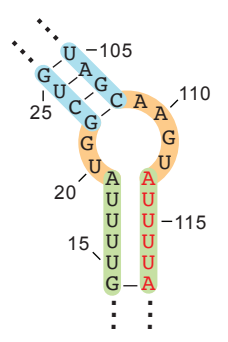

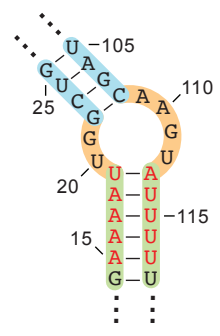


bioRxiv preprint doi: https://doi.org/10.1101/2020.05.21.102756; this version posted May 21, 2020. The copyright holder for this preprint (which

A was not certified by peer review) is the author/funder, who has granted bioRxiv a license to display the preprint in perpetuity. It is made

A

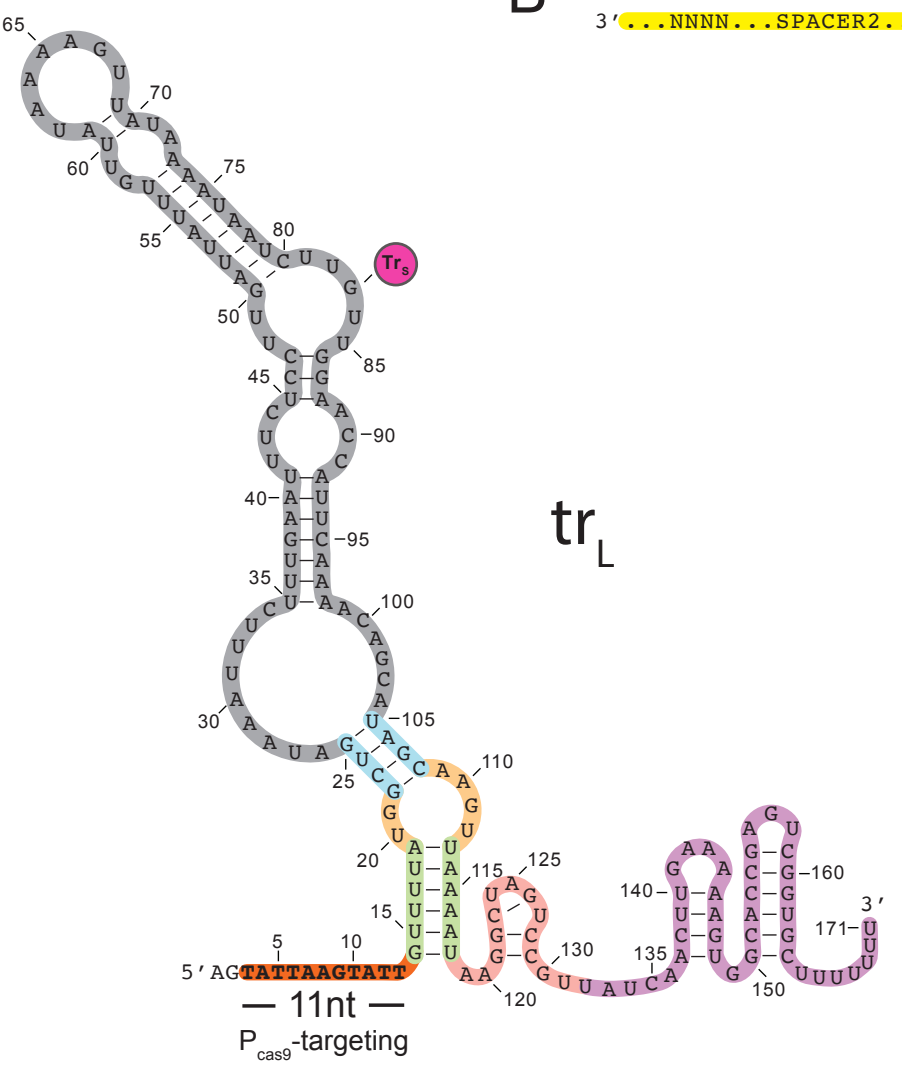

$E$

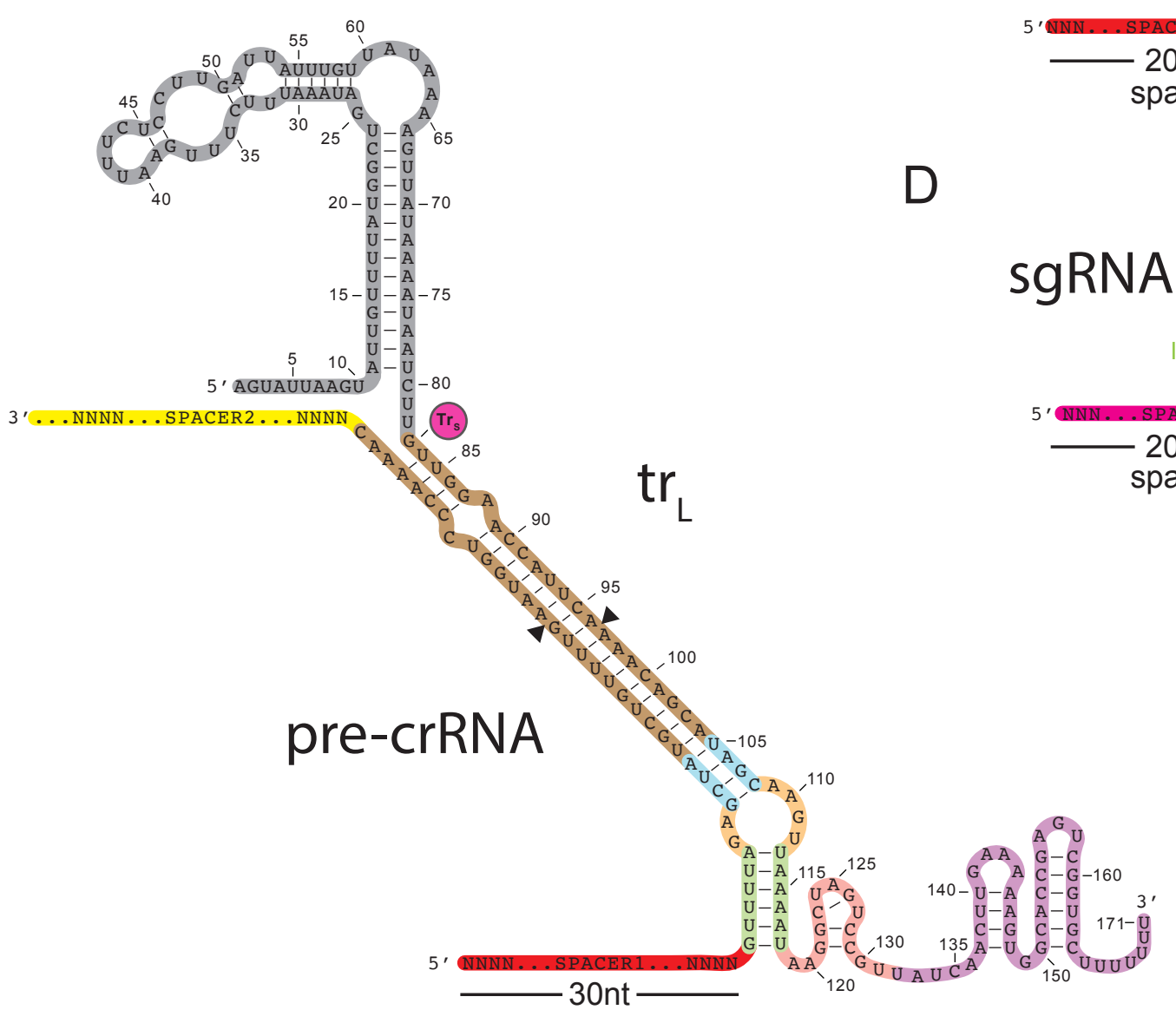

Fig. S6
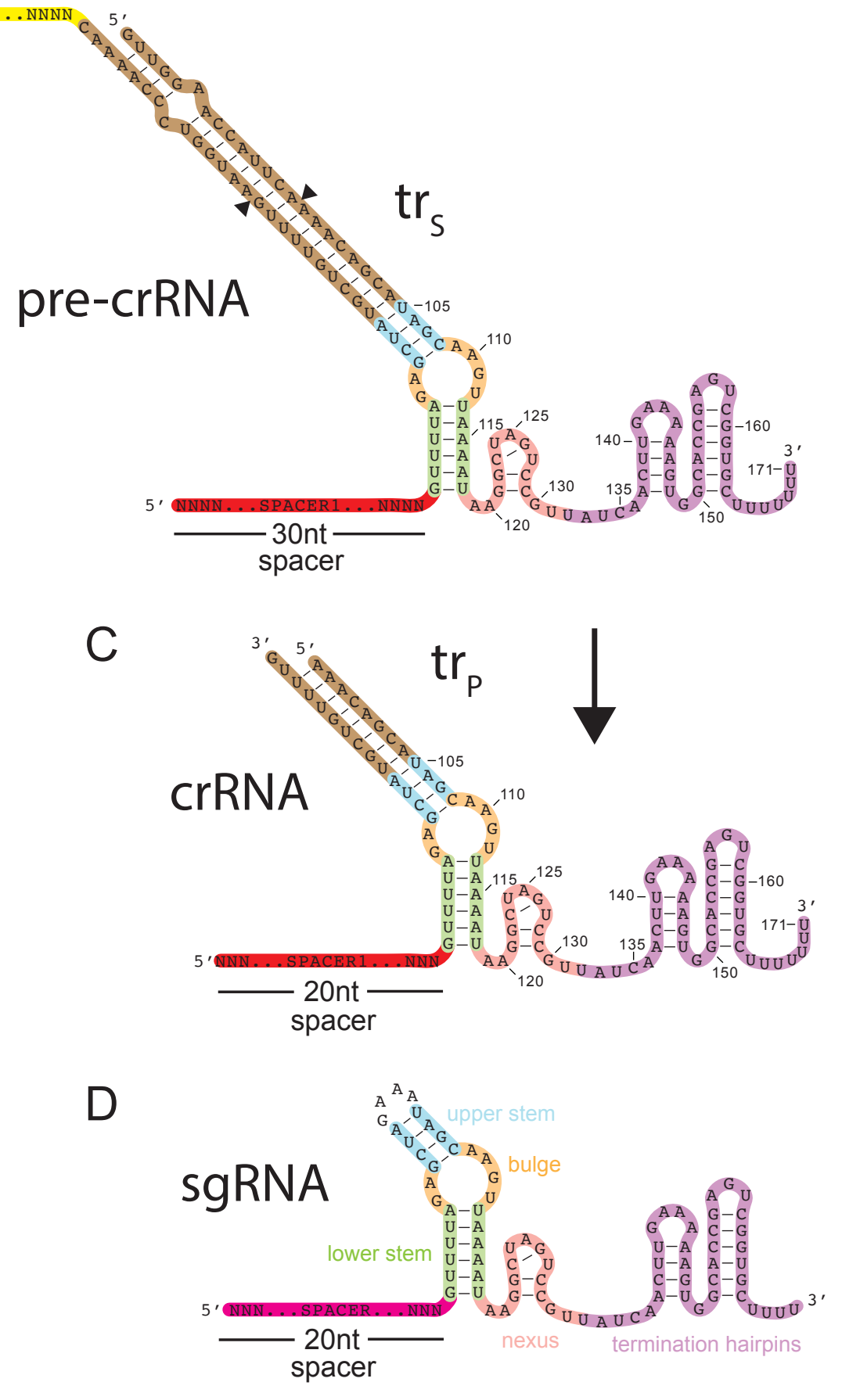
bioRxiv preprint doa: https://doi.org/10.1101/2020.05.21.102756; this version posted May 21, 2020. The copyright holder for this preprint (which

was not certified by peer review) is the author/funder, who has granted bioRxiv a license to display the preprint in perpetuity. It is made

Fig. S7

A available under aCC-BY-NC 40 International license.
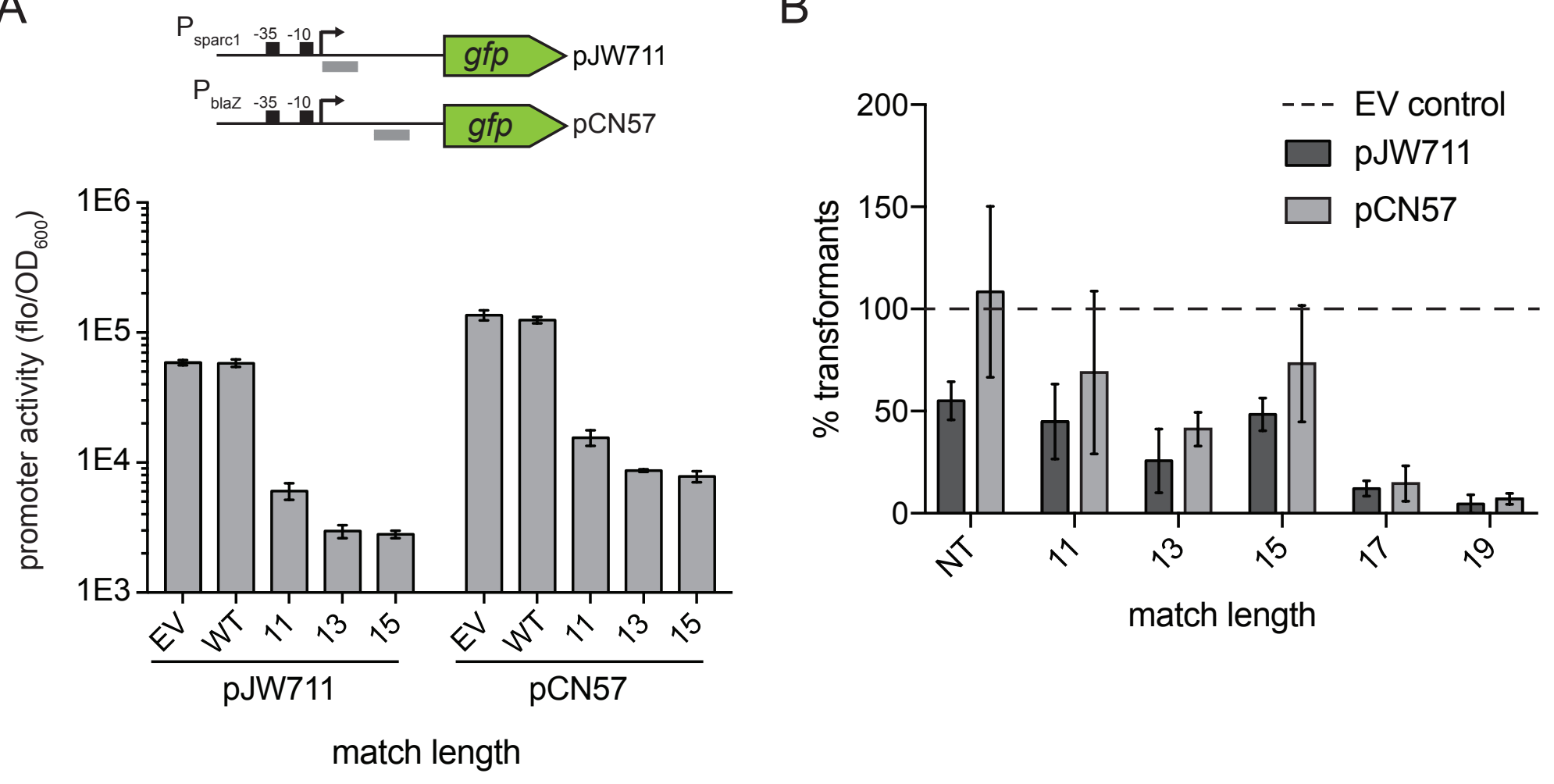

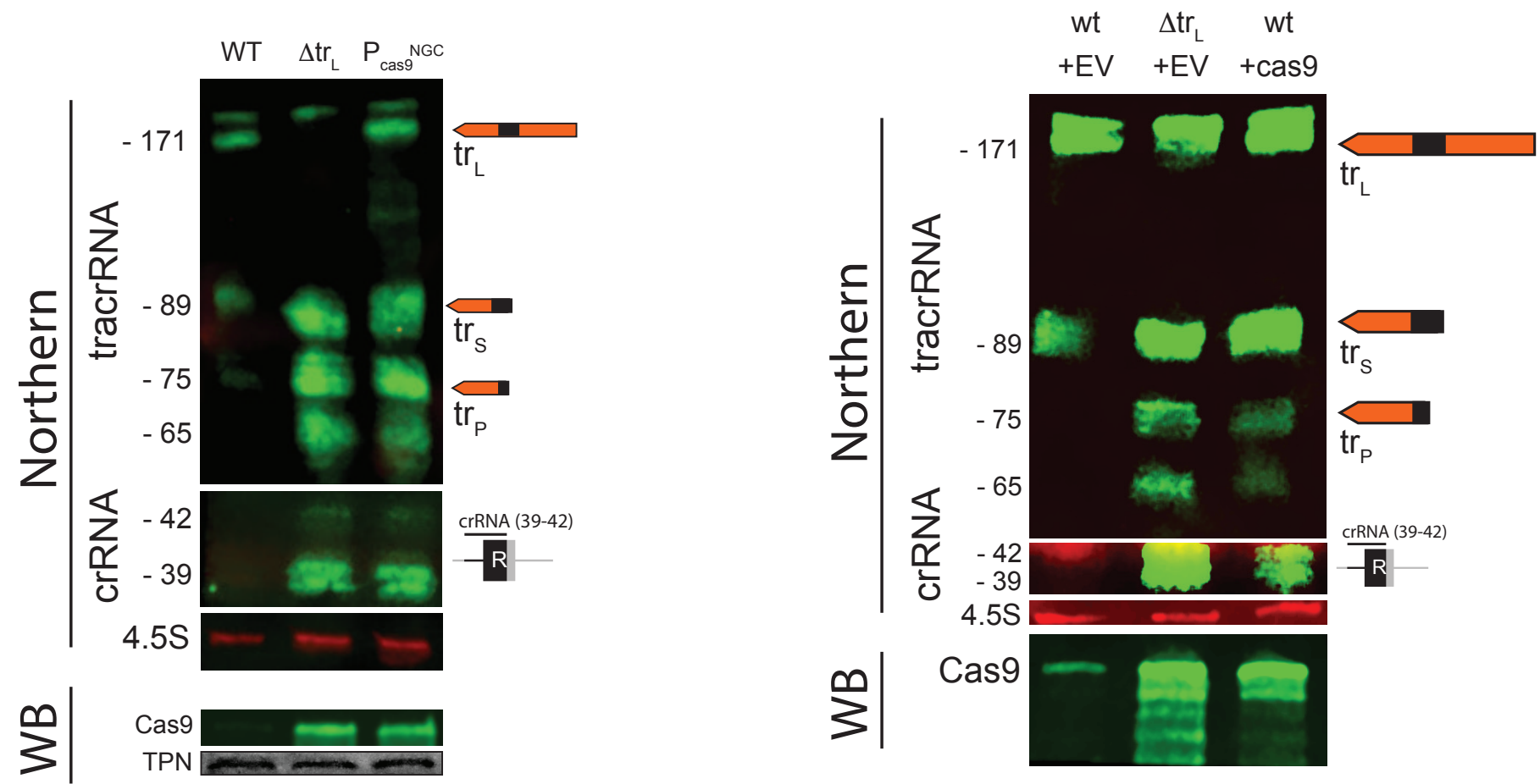

C

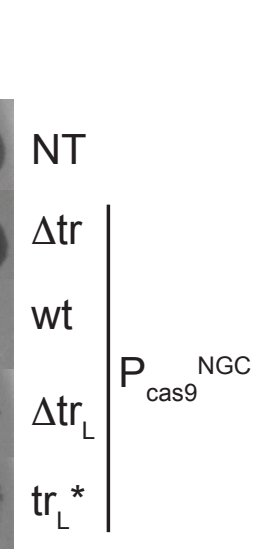

D
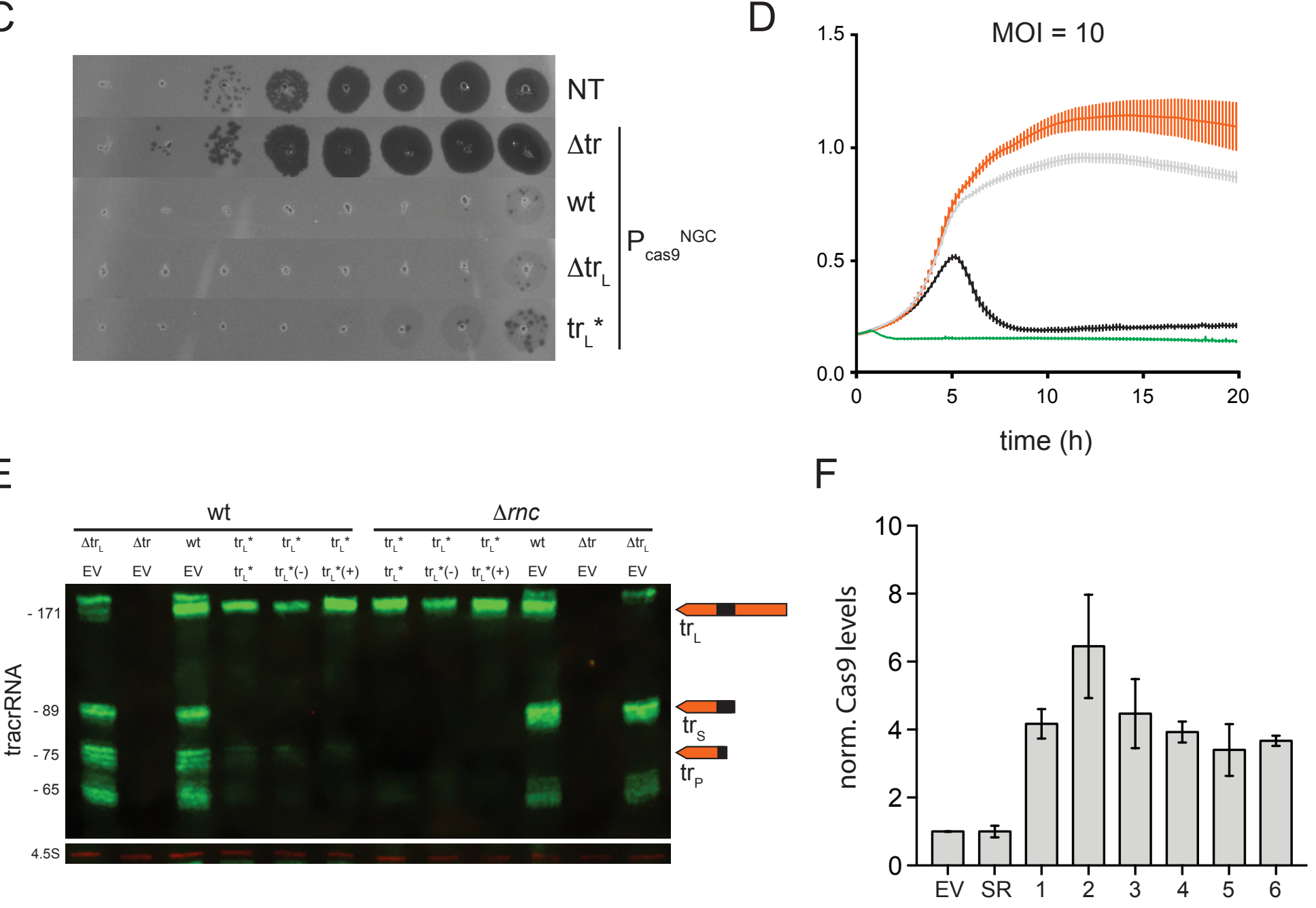
bioRxiv preprint doi: https://doi.org/10.1101/2020.05.21.102756; this version posted May 21, 2020. The copyright holder for this preprint (which

was not certified by peer review) is the author/funder, who has granted bioRxiv a license to display the preprint in perpetuity. It is made available under aCC-BY-NC 4.0 International license.

Fig. S9

Sequence Logo

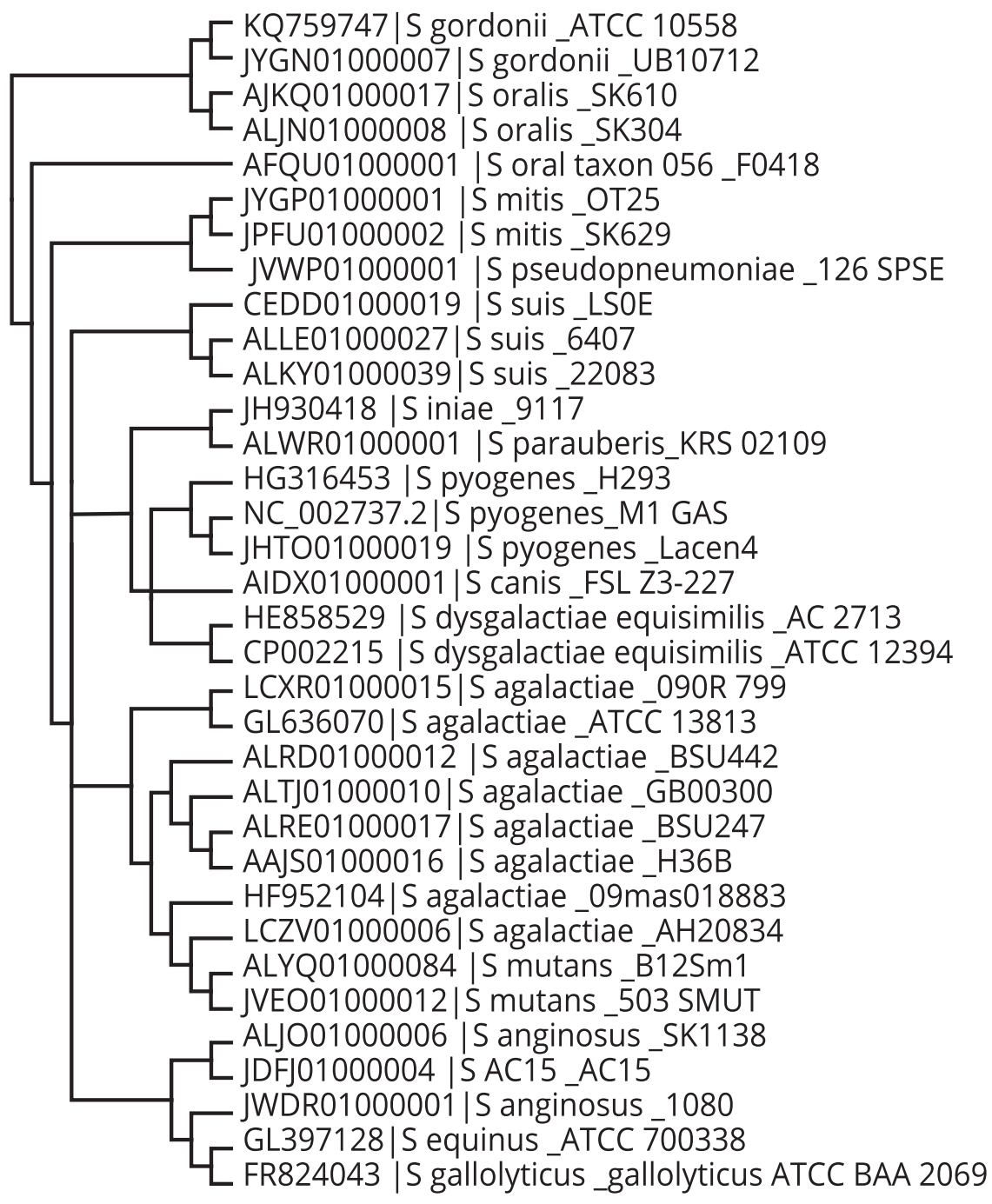

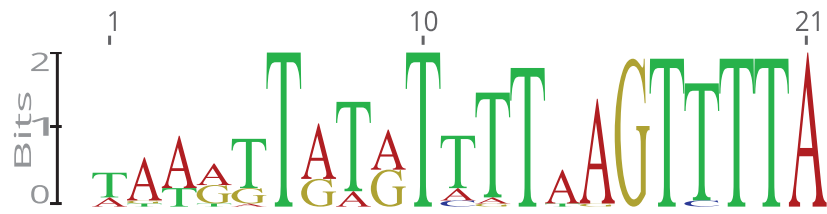

TAAGTTATATTTT-AGTTTTA TAAGTTATATTTT-AGTTTTA GTTATATTTT-AGTTTTA AAGTTATATTTT-AGTTTTA AGTTGTATTTT-AGTTTTA GTTGTATTTT-AGTTTTA GTTGTATTTT-AGTTTTA AAGTTATATTTT-AGTTTTA TTTGTATTT- - AGTTTTA GATTTATATTT--AGTTTTA ATTTGTATTT- - AGTTTTA TAATTGTATTAT-GGTTTTA TATTGTGTTAT-AGTTTTA TATTAAGTATT - - GTTTTA TATTAAGTATT- - GTTTTA TATTAAGTATT- - GTTTTA TATTATGTAGT- - GTTTTA TATTAAGTATT- - GTTTTA TATTAAGTATT- - GTTTTA TAAAGTATGTTTTAAGTTTTA TAAAGTATGTTTTAAGTTTTA TAAAGTATGTTTTAAGTTTTA TAAAGTATGTTTTAAGTTTTA TAAAGTATGTTTTAAGTTTTA TAAAGTATGTTTTAAGTTTTA TAAAGTATGTTTTAAGTTTTA TAAAGTATGTTTTAAGTTTTA ATATATTTTAAGTCTTA ATATATTTTAAGTCTТA AAAGTTGTATCTT-AGTTTTA AAAGTTGTATCTT-AGTTTTA AAAGTTGTATCTT-AGTTTTA TTATGTTTTTAGTTTTA

\footnotetext{
AG TIGTA TITI-A GI LSTA
} 
Fig. S10

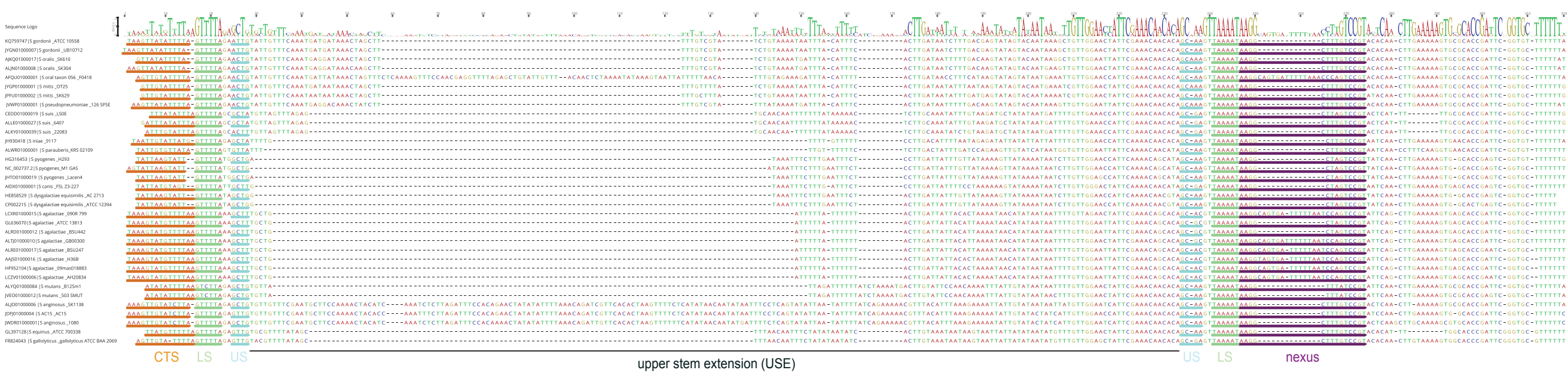


Fig. S11

A

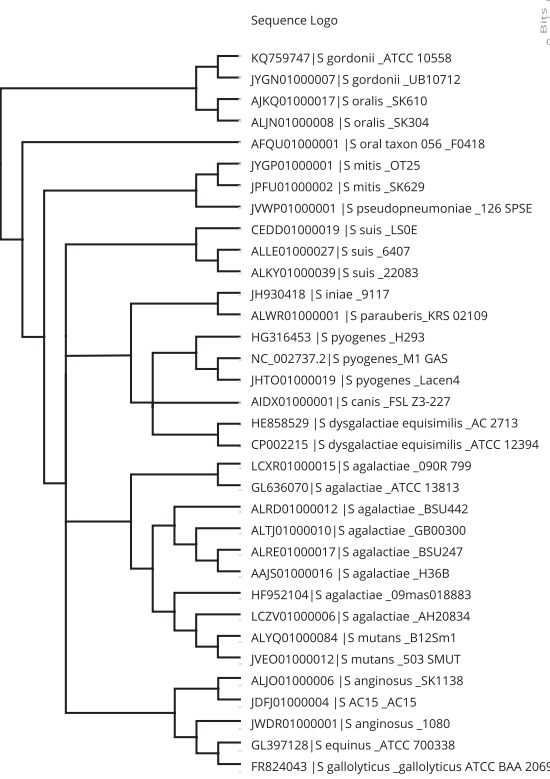

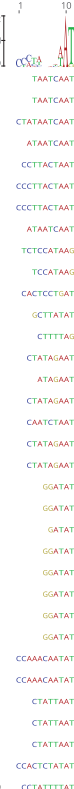

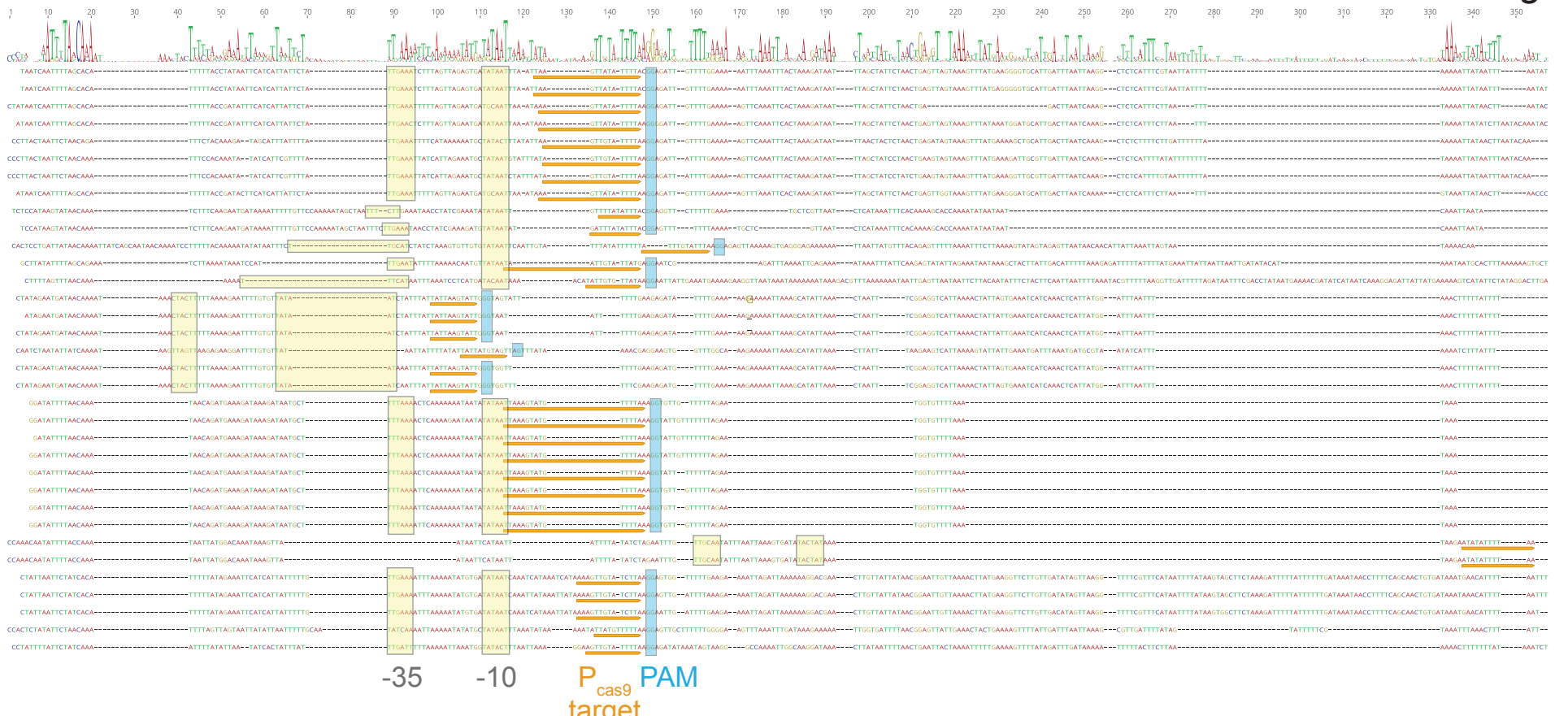

B

Sequence Logo
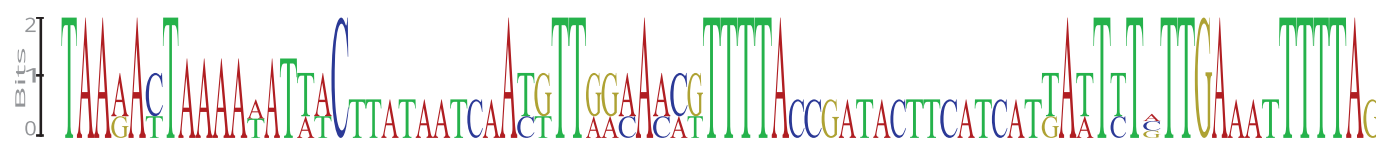

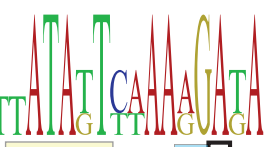

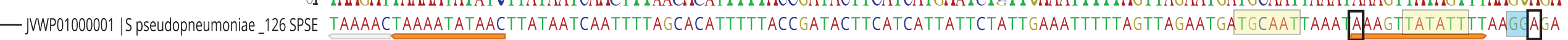

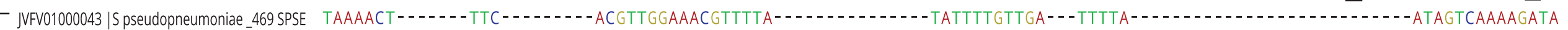
JUQX01000061 S S pseudopneumoniae_843 SPSE TAAGATTAAAAAATTAC - - - ATGTTGAAAACGTTTTA-

$$
\text { LS CTS } \quad-10 \quad+1 \mathrm{P}_{\text {cas }} \text { target }
$$

$+1$

Sequence Logo

CP001129 |S equi zooepidemicus_MGCS10565 JH930418 |S iniae_9117

L ALWR01000001 |S parauberis_KRS 02109 HG316453 |S pyogenes_H293 NC_002737.2|S pyogenes_M1 GAS -JHT001000019 |S pyogenes_Lacen4 _AIDX01000001 |S canis_FSL Z3-227 - HE858529 |S dysgalactiae equisimilis_AC 2713 CP002215 |S dysgalactiae equisimilis_ATCC 12394

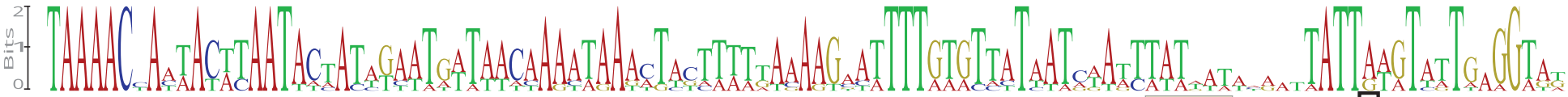

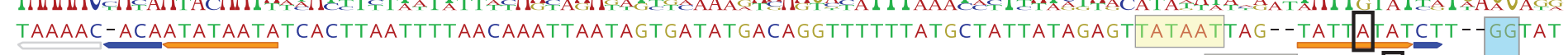

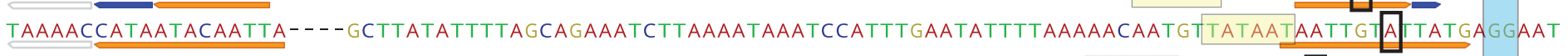

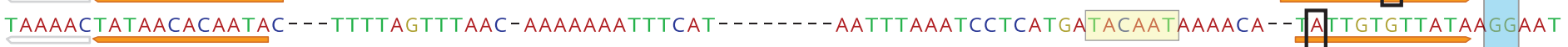
TAAAAC-AATACTTAATACTATAGAATGATAACAAAATAAACTACTTTTTAAAAGAATTTTGTGTTATAATCTATT A - - - - - TATTAAGTATTG-GGTAG

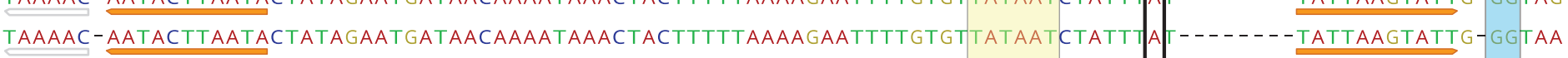

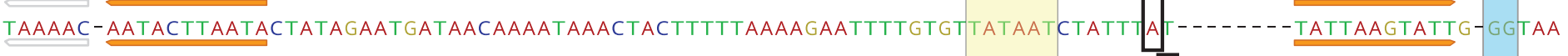

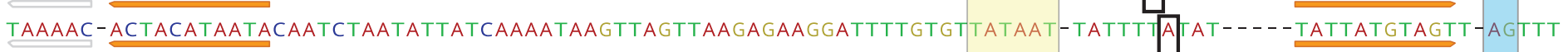
TAAAAC-AATACTTAATACTATAGAATgATAACAAAATAAACTACTTTTTAAAAGAATTTTGTGTTATAAT-AAATT A A -

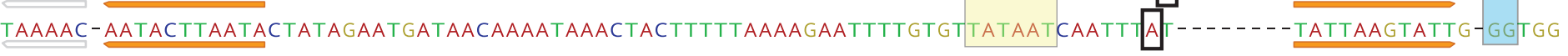
LS CTS cas9 target PAM 